EPJ Web of Conferences 104, 01004 (2015)

DOI: $10.1051 /$ epjconf/201510401004

(C) Owned by the authors, published by EDP Sciences, 2015

\title{
Small angle neutron scattering
}

\author{
Fabrice Cousin
}

Laboratoire Léon Brillouin, CEA-CNRS, CEA-Saclay, 91191 Gif-sur-Yvette, France

\begin{abstract}
Small Angle Neutron Scattering (SANS) is a technique that enables to probe the 3-D structure of materials on a typical size range lying from $\sim 1 \mathrm{~nm}$ up to $\sim$ a few $100 \mathrm{~nm}$, the obtained information being statistically averaged on a sample whose volume is $\sim 1 \mathrm{~cm}^{3}$. This very rich technique enables to make a full structural characterization of a given object of nanometric dimensions (radius of gyration, shape, volume or mass, fractal dimension, specific area...) through the determination of the form factor as well as the determination of the way objects are organized within in a continuous media, and therefore to describe interactions between them, through the determination of the structure factor. The specific properties of neutrons (possibility of tuning the scattering intensity by using the isotopic substitution, sensitivity to magnetism, negligible absorption, low energy of the incident neutrons) make it particularly interesting in the fields of soft matter, biophysics, magnetic materials and metallurgy. In particular, the contrast variation methods allow to extract some informations that cannot be obtained by any other experimental techniques. This course is divided in two parts. The first one is devoted to the description of the principle of SANS: basics (formalism, coherent scattering/incoherent scattering, notion of elementary scatterer), form factor analysis $(\mathrm{I}(\mathrm{q} \rightarrow 0)$, Guinier regime, intermediate regime, Porod regime, polydisperse system), structure factor analysis ( $2^{\text {nd }}$ Virial coefficient, integral equations, characterization of aggregates), and contrast variation methods (how to create contrast in an homogeneous system, matching in ternary systems, extrapolation to zero concentration, Zero Averaged Contrast). It is illustrated by some representative examples. The second one describes the experimental aspects of SANS to guide user in its future experiments: description of SANS spectrometer, resolution of the spectrometer, optimization of spectrometer configurations, optimization of sample characteristics prior to measurements (thickness, volume, hydrogen content...), standards measurements to be made and principle of data reduction.
\end{abstract}

Résumé. (La Diffusion de Neutrons aux petits angles (DNPA) est une technique permettant de sonder la structure 3-D de matériaux sur une gamme typique de taille comprise entre $\sim 1 \mathrm{~nm}$ ) et $\sim$ quelques $100 \mathrm{~nm}$, les informations obtenues étant statistiquement moyennées sur la taille de l'échantillon dont le volume est d'environ $1 \mathrm{~cm}^{3}$. C'est une technique très puissante car elle permet de faire une caractérisation complète de la structure d'un objet donné de dimensions nanométriques (rayon de giration, forme, volume, masse, dimension fractale, surface spécifique...) grâce à la détermination du facteur de forme, ainsi que la détermination de la manière dont les objets sont organisés au sein d'un milieu continu, et donc de décrire les interactions entre les objets, par la détermination du facteur de structure. Les propriétés spécifiques de neutrons (possibilité de moduler le contraste par substitution isotopique, sensibilité au magnétisme, absorption négligeable, faible énergie des neutrons incidents) la rendent

This is an Open Access article distributed under the terms of the Creative Commons Attribution License 4.0, which permits unrestricted use, distribution, and reproduction in any medium, provided the original work is properly cited. 
particulièrement intéressante dans les domaines de la matière molle, de la biophysique, des matériaux magnétiques et de la métallurgie. En particulier, les techniques de variation de contraste permettent d'extraire des informations qui ne peuvent être obtenues par aucune autre technique expérimentale. Ce cours est divisé en deux parties. La première est consacrée à la description du principe de la DNPA : bases de la technique (formalisme, diffusion cohérente/diffusion incohérente, notion de diffuseur élémentaire, analyse du facteur de forme $(\mathrm{I}(\mathrm{q} \rightarrow 0)$, régime de Guinier, régime intermédiaire, régime de Porod, système polydisperse), analyse du facteur de structure $\left(2^{\text {nd }}\right.$ coefficient du Viriel, équations intégrales, caractérisation d'agrégats), et méthode de variation de contraste (comment créer un contraste dans un système homogène, annulation de contraste dans un système ternaire, extrapolation à concentration nulle, contraste moyen nul). La seconde partie décrit les aspects expérimentaux de la DNPA afin de guider l'utilisateur dans ses expériences futures: description d'un spectromètre de DNPA, résolution d'un spectromètre, optimisation des configurations du spectromètre, optimisation des caractéristiques de l'échantillon avant les mesures (épaisseur, volume, teneur en hydrogène...), mesures standards à effectuer et principe de la réduction des données.

\section{Introduction}

Small Angle Neutron Scattering (SANS) is a technique that enables to probe the structure of materials on a typical size range lying from $\sim 1 \mathrm{~nm}(10 \AA)$ up to $\sim$ a few $100 \mathrm{~nm}(1000 \AA)$, a superior limit that can be extended of almost a decade by specific geometries of measurements enabling to reach very small q's using Ultra SANS and Very SANS). For a system containing typically a given type of objects (nanoparticles, polymers, pores, proteins...) in a continuous media (solvent, organic or inorganic matrix), SANS enables to describe the object (gyration radius, shape, volume, mass, fractal dimension, specific area...) and the way the objects are organized within the continuous media (aggregated or homogeneously dispersed...), and therefore to describe interactions between them. Whatever the geometry, it is possible to access to the specific area of scattering objects in the sample, which is less known but very useful as for example the specific area of porous media. Since it is a scattering technique, the obtained information is statistically averaged on the whole sample that has a volume of typically $\sim 1 \mathrm{~cm}^{3}$, which does not allow to observe a specific given detail of a sample, as microscopy techniques do. The information is also obviously averaged on the characteristic time of measurement, that lies between a few minutes and several hours.

Given the actual rather weak brightness of the neutron sources, whether they are reactors of spallation sources, one commonly choose to work with a poor experimental resolution (typically $\delta \mathrm{q} / \mathrm{q} \sim$ $10 \%$ ) to keep a correct signal-to-noise ratio. This is different from its counterpart scattering technique with X-Rays (Small Angle X-Rays Scattering) that probes exactly the same q-range, and therefore the same spatial characteristic sizes, for which the resolution is excellent. Given that SAXS may often be accessed in simple laboratories and not necessarily requires large facilities, SAXS appears to be $a$ priori preferable to SANS. However neutrons have some unique specificities that make them a better choice than $\mathrm{X}$-rays in various cases:

- First, the neutron-matter interaction directly occurs between the neutron and the nuclei of atoms, and not with the electronic cloud, as for X-rays. The amplitude of such interaction, id est the coherent scattering length, is tabulated and varies randomly from one atom to another along the periodic table of elements (even within isotopes ) and can be either positive or negative, contrarily to the case of X-rays for which is always positive and proportional to the number of electrons. It appears that the of hydrogen ${ }^{1} \mathrm{H}$ has an opposite sign and very different value than the ones of all other atoms constitutive organic molecules (C,O,N..) and, last but not least, than the one from deuterium ${ }^{2} \mathrm{H}$ (D). In SANS, one probes larger sizes than the atoms, and the relevant parameter for describing the interaction of a molecule with neutrons is then the Scattering Length 
Density (SLD) which is the volume average of all the coherent length of its constitutive atoms. The SLD of a given organic molecule is thus essentially dependent on its content on hydrogen atoms. In particular, the replacement of some hydrogen atoms of a molecule by deuterium atoms will strongly modify its SLD without altering much its physical properties. This unique properties of neutrons opens the way to contrast variation experiments where some molecules are labeled by deuterium to create a neutron contrast in the system. It is also possible to continuously tune the SLD of a solvent in a complex system by mixing hydrogenated and deuterated solvents in order to match the SLD of a component, making it invisible in the measured scattering curve.

- As they bear a spin $\pm 1 / 2$, the second important specificity of neutrons is their ability to interact with magnetic moments of molecules. In this course, only the nuclear interaction will be considered. The case of scattering by magnetic systems can be found elsewhere (1) (2).

- The third important property of neutrons is their very weak absorption by matter (they do not interact with electric fields...) that can be neglected in general, except in cases when one probes materials containing one of the few atoms that have a huge neutron absorption cross-section (boron, gadolinium, lithium,...). This enables to make in situ experiments in various samples environments, in particular within devices made of metallic components (pressure cells, etc.).

- The fourth and last important characteristic of neutrons is their weak energy. After their thermalization at the wavelengths required for experiments, they have indeed an energy of a few meV. SANS experiments are thus absolutely not destructive, which is of great importance in biology or for the expertise of industrial devices.

Despite of its apparent simplicity, SANS is a very rich technique and it is not possible to cover all of its aspects in a single introduction course. There are several books for those who want to go beyond that cover the different aspects of SANS, from "historical" (3) ones up to more recent ones (4) (5) (6). For English Readers, an exhaustive selection of useful reviews or textbooks on the topic can be found on the SAS Portal website (7). Within this selection, we suggest the recent one from B. Hammouda because it is especially complete and is available free of charge on internet (5). For the French readers, more information can also be found in the collection SFN (8), in particular in the issues "Diffusion de Neutrons aux Petits Angles", "Neutrons et Magnétisme" "Surfaces, Interfaces, Milieux Confinés par Diffusion de Neutrons" and "Neutrons et Matière Molle".

This course is divided in two main parts. In the first part, I will present the principles of SANS ("small angle" approximation, form factor, structure factor etc.), in the second part how it is possible to realize a SANS experiment from a practical point of view (spectrometers, data reduction, samples, accessibility to neutrons beams...). I have remarked that the theoretical aspects are generally exhaustively treated in textbooks but not the practical ones, which prompted me to emphasize on these last aspects in the second part. I hope that it may be useful for future users.

\section{Principle of SANS}

\subsection{Principle}

In a scattering experiment, the sample is placed in the incident neutron beam, considered as a planar monochromatic wave of wavelength $\lambda$, which is described by $\psi_{i}(x, t)=\psi_{0} e^{i\left(\omega t-k_{i} x\right)}$ where $\omega$ is the pulsation and $\mathrm{k}_{\mathrm{i}}=2 \pi / \lambda$ is the norm of the wavevector.

When the wave interacts with a given atom of the sample, it is scattered over all directions. The scattered wave is thus spherical. In the following, we will assume that the scattering is elastic, $i d$ est there is no exchange of energy between the neutron and the atom. The scattered wave and incoming wave have the same wavelength. The probability of a neutron to be scattered by an atom is proportional to the area $\sigma$ of a characteristic surface of the interaction between this atom and the neutron called the scattering cross-section. With hands, it corresponds to the surface of the atom probed by the 


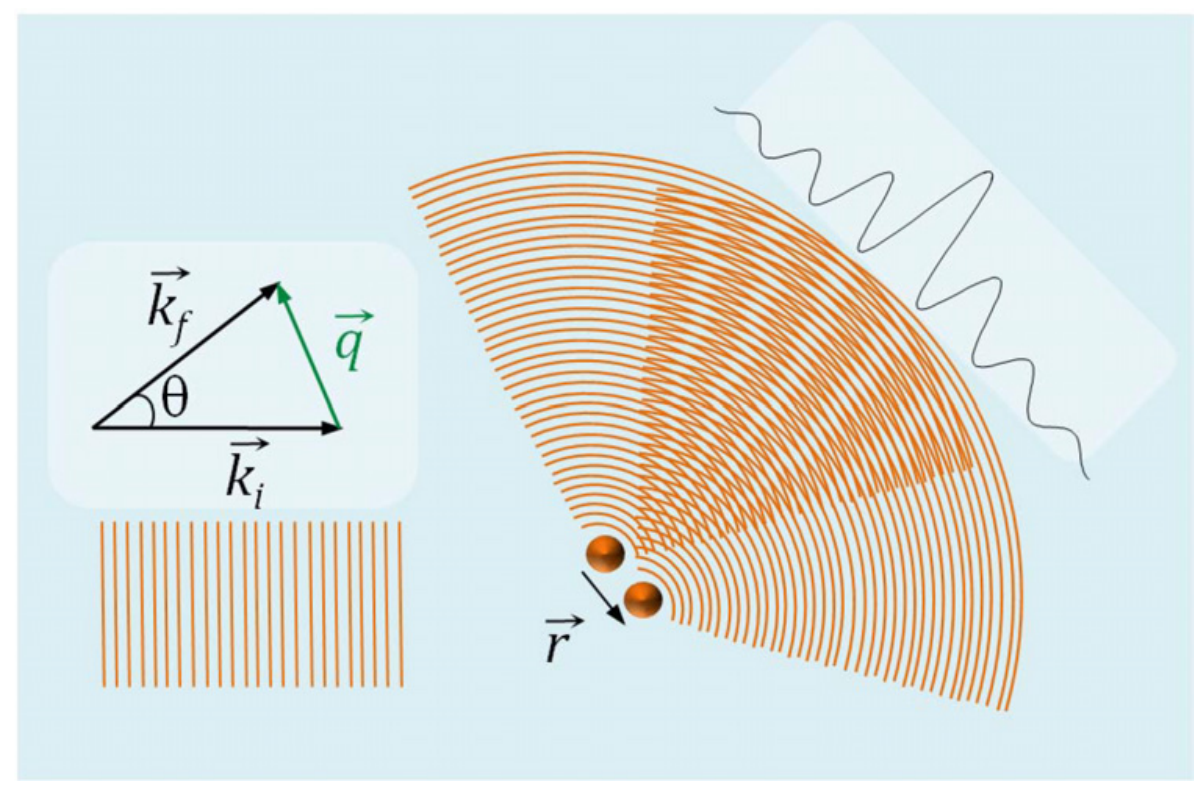

Figure 1. Principle of scattering and definition of scattering vector $\vec{q}$. Interferences between two spherical waves scattered by two atoms separated by $\vec{r}$ in real space. The interferences spectrum depends on the distance between atoms and therefore of its structure.

neutron wave. The intensity of the wave scattered over $4 \pi$ is then $\mathrm{I}_{\text {tot }}=\Phi_{0} \sigma$ (in neutrons/sec) if $\Phi_{0}$ is the incoming neutrons flux in neutrons/sec. The scattering cross section can thus be written like $\sigma=$ $b^{2}$, where $\mathbf{b}$ is the scattering length that is characteristic of the range of the interaction between the neutron and the atom. Note that neutrons interact with the nuclei of the atom, on the contrary to photons which interact with the electronic cloud of the atoms. The order of the range of the interaction is then of the order of the nuclei' size and the typical values of $b$ lie in the femtometer range $\left(10^{-15} \mathrm{~m}\right)$. This is much smaller than the typical dimension of the atoms (angstroem, $\sim 10^{-10} \mathrm{~m}$ ). The interaction can thus be considered as punctual, contrary to the case of photons, making the formalism describing neutrons rather simple. In particular, the interaction with a scatterer does not depend on the scattering by the other scatterers (Born approximation).

The intensity $\mathrm{I}_{\text {det }}$ collected by a detector of surface $S$ located at the distance D from the scattering atom is then proportional to the solid angle $\Omega=S / \mathrm{D}^{2}$ of the detector with respect to the atom $\mathrm{I}_{\text {det }}=\Phi_{0} \sigma \Omega=\Phi_{0}(\mathrm{~b} / \mathrm{D})^{2} S$. Such intensity is the square of the scattered spherical wave at $\mathrm{D}$ that writes:

$$
\psi=\psi_{0} \frac{b}{D} e^{i(\omega t-k D)}
$$

Let us now consider $n$ atoms that scatter $n$ spherical waves that will interfere. The whole scattered wave is the thus superposition of spherical waves scattered by each atom, corrected by a phase factor due the phase difference of the incident wave to reach the position of another nucleus at $\vec{r}$ from the origin*. It is illustrated in Fig. 1 for the two atoms case. The phase shift $\Delta \Phi$ writes:

$$
\Delta \Phi=\vec{r} \cdot\left(\vec{k}_{f}-\vec{k}_{i}\right)
$$

\footnotetext{
*We consider implicitly within the whole theoretical part that a neutron is scattered once and only once inside the sample.
} 
It is then convenient to introduce the scattering vector $\vec{q}=\vec{k}_{f}-\vec{k}_{i}$.

Its norm is: $\|\vec{q}\|=q=\frac{4 \pi}{\lambda} \sin \left(\frac{\theta}{2}\right)$

When the detector is placed at a distance $\mathrm{D}$ that is much larger than the sample size, which is practice always the case (see part 3 ), the scattering angle $\theta$ is the same for all the atoms. The final scattered wave is the sum of the scattered waves by all atoms:

$$
\psi=\frac{\psi_{0}}{D} \times \sum_{i=1}^{n} b_{i} e^{i \vec{q} \cdot \vec{r}_{i}}
$$

The neutron detector is not sensitive to the amplitude of the wave but to the intensity which is the square of the amplitude $I=\psi \cdot \psi^{*}=\left(\frac{\psi_{0}}{D}\right)^{2} \times \sum_{i}^{n} \sum_{j}^{n} b_{i} b_{j} e^{i \vec{q} \cdot\left(\vec{r}_{i}-\vec{r}_{j}\right)}$. In order to get rid of the term in $\left(\frac{\psi_{0}}{D}\right)^{2}$, we define the differential scattering cross section $\frac{d \sigma}{d \Omega}(q)$, that will be denoted further in part 2 as $\Sigma(\mathrm{q})$.

$$
\Sigma(q)=\frac{d \sigma}{d \Omega}(q)=\sum_{i}^{n} \sum_{j}^{n} b_{i} b_{j} e^{i \vec{q} \cdot\left(\vec{r}_{i}-\vec{r}_{j}\right)}
$$

This differential scattering cross section is a measurement of the probability to find an atom with a coherent length $b_{i}$ and another atom of coherent length $b_{j}$ separated by a vector $\overrightarrow{\mathbf{r}}_{\mathbf{i}}-\overrightarrow{\mathbf{r}}_{\mathbf{j}}$.

It is homogenous to a surface $\left(\mathrm{cm}^{2}\right)$. Experimentally, the measured intensity is the differential scattering cross section per unit volume:

$$
I(q)\left(\mathrm{cm}^{-1}\right)=\frac{1}{v} \frac{d \sigma}{d \Omega}(q)
$$

where $\mathrm{V}$ is the volume of the sample.

Theoretically, the scattering amplitude of the differential scattering cross section $A(\vec{q})$ (such as $\Sigma(q)=\langle A(\vec{q}) A(\overrightarrow{-q})\rangle$ is the Fourier transform of the density $\rho(\vec{r})$ of the system of $\mathrm{n}$ atoms, weighted by the scattering length $b_{i}$ of the atom located in $\vec{r}_{i}$.

$$
\rho(\vec{r})=\sum_{i=1}^{n} \rho_{i}(\vec{r})=\sum_{i=1}^{n} \delta\left(\vec{r}-\vec{r}_{i}\right)
$$

where $\delta$ is the Dirac function.

Then $A(\vec{q})$ writes:

$$
A(\vec{q})=\int_{V} e^{i q \cdot r} \cdot d_{3} \vec{r} \cdot \sum_{i} b_{i} \delta\left(\vec{r}-\vec{r}_{i}\right)
$$

In the case where all the atoms have the same scattering length $\left(b_{i}=b\right.$, when they are all of the same isotope, without spin, see part 2.2), $\Sigma(\mathrm{q})$ can be written like:

$$
\Sigma(q)=\sum_{i, j}^{n, n} b^{2}\left\langle e^{i \vec{q}\left(\vec{r}_{i}-\vec{r}_{j}\right)}\right\rangle=b^{2}\langle\rho(\vec{q}) \rho(-\vec{q})\rangle=b^{2} \int d_{3} \vec{r} \cdot e^{i \vec{q} \vec{r}} g(\vec{r})
$$

where the brackets stand for an average over all positions of atoms. $\boldsymbol{g}(\overrightarrow{\boldsymbol{r}})$ is the pair correlation function, id est the probability to have two atoms separated by $\vec{r}$. 


\subsection{Coherent scattering/incoherent scattering}

In the previous part, we have implicitly considered that a given atomic species has a single scattering length $\mathrm{b}_{i}$. In practice, this is not the case for two reasons: $(i)$ first, since neutrons interact with nuclei of atoms, the coherent scattering length is different from one isotope to another. This apparent drawback can be turned into an advantage and is the foundation of the variation contrast method that will be developed in the following; (ii) Second, the interaction of neutrons with atoms also takes place between the spin $1 / 2$ of neutrons and the nuclear spin of atom when this latter is non-zero, id est the scattering length of an atom depends on its spin state.

Thus, even when a sample is only composed of atoms of the same chemical composition with uncorrelated spatial positions (like a "sea" of atoms), the neutrons see a mixture of scatterers with different scattering lengths whom positions in space fluctuate but are not correlated. Such fluctuations contribute to the scattered intensity. It is then necessary to take into account the random probability of occupancy of an atom by a given isotope in a given spin state and to make averages on the corresponding scattering lengths. The scattered intensity writes then:

$$
\Sigma(\vec{q})=n\left(\left\langle b_{2}\right\rangle+\langle b\rangle^{2}\right)+\langle b\rangle^{2} \sum_{i}^{n} \sum_{j}^{n} e^{i \vec{q} \cdot\left(\vec{r}_{i}-\vec{r}_{j}\right)}
$$

Or

$$
\Sigma(q)=n b_{i n c}^{2}+b_{c o h}^{2} \sum_{i}^{n} \sum_{j}^{n} e^{i \vec{q} \cdot\left(\vec{r}_{i}-\vec{r}_{j}\right)}
$$

$b_{c o h}^{2}$ is the coherent scattering cross-section and $b_{i n c}^{2}$ is the incoherent scattering cross-section, whose contribution to the overall scattered intensity is q-independent. In SANS, it is thus a flat background that has to be subtracted (see part 3). In the following, only the coherent differential scattering cross section $\Sigma_{\text {coh }}(\mathrm{q})$ will be considered.

\subsection{Spatial resolution/Scattering length density}

\subsubsection{Spatial resolution}

A very important characteristic of SANS is a typical scale that is probed. Indeed the amplitudes scattered by two points will only interfere constructively when the distance is smaller than $\left|\vec{r}_{i}-\vec{r}_{j}\right|$ than $q^{-1}$ because:

$$
\begin{aligned}
& \exp \left(i \vec{q} \cdot\left(\vec{r}_{i}-\vec{r}_{j}\right)\right) \approx 0 \quad \text { when }\left|\overrightarrow{\mathrm{r}}_{\mathrm{i}}-\overrightarrow{\mathrm{r}}_{\mathrm{j}}\right|>\mathrm{q}^{-1} \\
& \exp \left(i \vec{q} \cdot\left(\vec{r}_{i}-\vec{r}_{j}\right)\right) \approx 1 \quad \text { when }\left|\overrightarrow{\mathrm{r}}_{\mathrm{i}}-\overrightarrow{\mathrm{r}}_{\mathrm{j}}\right|<\mathrm{q}^{-1}
\end{aligned}
$$

Two essential rules of scattering can be derived from these equations: $(i)$ at a spatial scale lower than $\mathrm{q}^{-1}$, the waves scattered by the different atoms are in phase and their amplitudes add themselves while at a spatial scale larger than $\mathrm{q}^{-1}$, the intensities of scattered waves add themselves; (ii) when one looks at the coherent scattered intensity around a given angle for a given q, one probes the structure of the system at a spatial scale with a typical order of resolution of $\mathrm{q}^{-1}$.

In practice, this means that only the fluctuations of scattering length of the order of $q^{-1}$ are probed in a given range of $q$. With hands, the modulus of the scattering vector acts like a magnifying glass that enables to determine the structure at the $\mathbf{q}^{-1}$ scale (Fig. 2). q values can be easily tuned by SANS by about three orders of magnitudes, lying typically between $\mathbf{1 0}^{-\mathbf{3}} \AA^{-\mathbf{1}}$ to $0.6 \AA^{-\mathbf{1}}$ on classical 
JDN 21

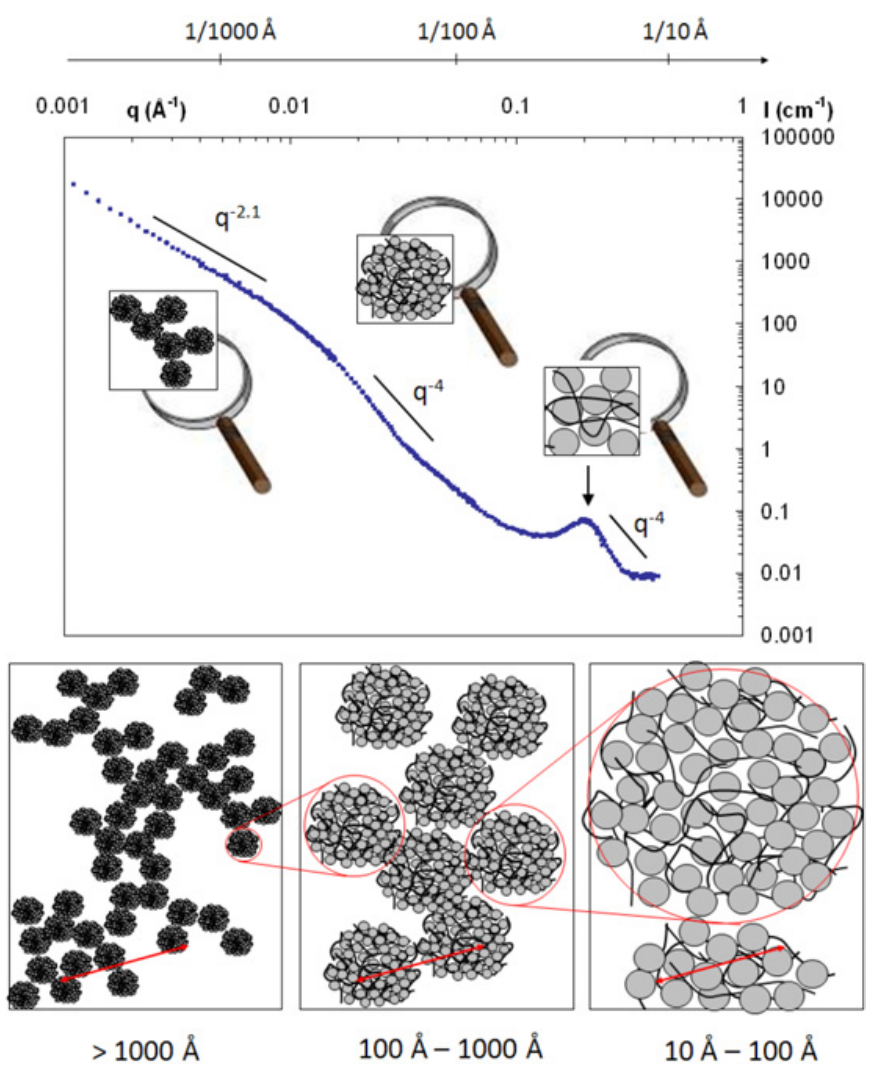

Figure 2. Principle of SANS illustrated by the scattering spectra of complex systems composed of spherical proteins and polyelectrolyte chains (from references (10) (12)). Each q range acts as magnifying glass: at larges $\mathrm{q}$, one probes the shape of proteins and their organization in the inner of the complexes $\left(\mathrm{q}^{-4}\right.$ decay and correlation peak at $\left.0.2 \AA^{-1}\right)$; at intermediates q, one probes the size and compactness of complexes ( $\mathrm{q}^{-4}$ decay and break of slope around $0.02 \AA^{-1}$ ), at small q, one probes the organization of complexes at large scales than the complexes size $\left(\mathrm{q}^{-2.1}\right.$ decay at small $\left.\mathrm{q}\right)$.

SANS machines, down to a few $\mathbf{1 0}^{-\mathbf{4}} \AA^{-\mathbf{1}}$ on specific Very SANS such as TPA at Laboratoire Léon Brillouin (9) and down to a few $\mathbf{1 0}^{-\mathbf{5}} \AA^{-\mathbf{1}}$ on Ultra SANS spectrometers.

\subsubsection{Notion of elementary scatterer/scattering length density}

From previous part it appears a very important specificity of SANS related to the relevant elementary scatterers (ES) that have to be considered in such experiment. Given the typical spatial scales that are probed ( $>5 \AA$ in direct space), a "small angle approximation" can be made: the elementary scatterers are no longer the atoms but the molecules. In others words, when the atoms are grouped in molecules whose sizes are small compared to $\mathrm{q}^{-1}$, the waves scattered by the different atoms are in phase and their amplitudes add themselves. The coherent scattering length of an elementary scatterer is the sum of the coherent scattering lengths of its constitutive atoms. For a given molecule, this coherent scattering length does obviously depend on the chemical composition but also on the respective contents of the different isotopes within the molecule.

Finally the relevant parameter to account for the probability of an elementary scatterer (ES) to scatter a neutron is its coherent scattering length per unit volume cell (Fig. 3). When there are no density 

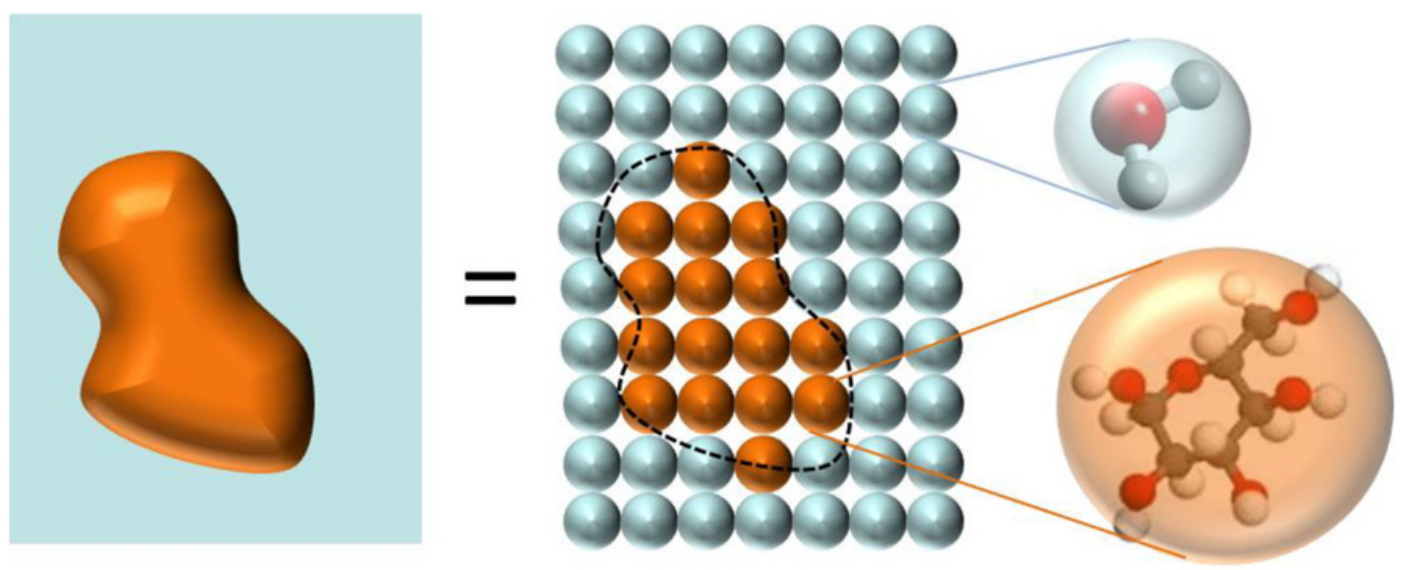

Figure 3. Notion of elementary scatterer in a case of an object in a continous phase. The object is made of elementary scatters that have all the same chemical composition, similarly as the continuous phase. Here the elementary scatterer of the object is a sugar unit while the elementary scatterer of the continous phase is a water molecule.

fluctuations within the ES, in the case of crystals or amorphous solids, this parameter is the coherent scattering length density (SLD) usually designed by $\rho$ (or sometimes by $\mathrm{N}_{\mathrm{b}}$ in neutron reflectivity) that writes:

$$
\rho=\frac{\sum_{i}^{n} b_{i}}{v}
$$

where the $b_{i}$ are the respective coherent scattering length off the constitutive atoms of the elementary scatterer and $v$ is its molar volume.

When the ES is a simple liquid like a common solvent, the question of possible density fluctuations at spatial scales lower than $\mathrm{q}^{-1}$ arises. However, at the spatial scales probed in SANS, a liquid is almost incompressible within the SANS q-range and the scattering length density can be obtained by Eq. (12), $v$ being easily obtained from the density of the liquid (see as an example the calculation of $\rho_{\text {water }}$ in part 3.21). Formally, thermodynamics indeed enables to demonstrate that the extrapolation to q equal to 0 of the density fluctuations $\Sigma(0)=b^{2}\left(\left\langle n^{2}\right\rangle-\langle n\rangle^{2}\right)$ of such liquid is proportional to its isothermal compressibility $\chi_{T}$ :

$$
\langle n\rangle k T \chi_{T}=v\left(\left\langle n^{2}\right\rangle-\langle n\rangle^{2}\right)
$$

where $k$ is the Boltzmann constant and T the temperature. This thermodynamic limit $\Sigma(0)$ is reached for the largest $q$ probed in SANS at around $q^{*} \sim 0.6 \AA^{-1}$. For $q<q^{*}$, these density fluctuations are negligible. Please note that in the case of mixture of liquids the concentration fluctuations may give rise to a noticeable contribution arising from compressibility in the scattered intensity.

\subsubsection{Mixture of elementary scatterers and contrast term}

Let us now consider a mixture of $\alpha=1,2, \cdots, m$ elementary scatterers of respective scattering lengths $\mathrm{b}_{\alpha}$ in a volume V. Equation (10) may be rewritten as:

$$
\Sigma(\vec{q})=\sum_{\alpha} n_{\alpha} b_{i n c}^{\alpha}+\sum_{\alpha, \beta}\left\langle b_{\alpha}\right\rangle\left\langle b_{\beta}\right\rangle \sum_{i, j}^{n_{\alpha}, n_{\beta}}\left\langle e^{i \vec{q} \cdot\left(\vec{r}_{i}^{\alpha}-\vec{r}_{j}^{\beta}\right)}\right\rangle
$$


The first term is the incoherent part and won't be considered in the following. The second part is often written:

$$
\Sigma(\vec{q})=\sum_{\alpha, \beta} b_{\alpha} b_{\beta} S_{\alpha \beta}(\vec{q})
$$

where the $S_{\alpha \beta}(\vec{q})$ are the partial structure factors of the different ES of the system. A given $S_{\alpha \beta}(\vec{q})$ is dependent only on the structure of the sample, id est it is the Fourier transform of the correlation function of the specie $\alpha$ with the specie $\beta$, but not on the contrast.

Let us now consider that the system is isotropic $\left(S_{\alpha \beta}(\vec{q})=S_{\alpha \beta}(\vec{q})\right)$. The mean number of elementary scatterers is $\left\langle n_{\alpha}\right\rangle$ and each of them has an elementary volume $v_{\alpha}=\frac{v}{\left\langle n_{\alpha}\right\rangle}$. Equation 13 can thus be generalized:

$$
\left\langle n_{\alpha}\right\rangle k T \chi_{T}=\sum_{\beta} v_{\beta} S_{\alpha \beta}(0)
$$

Or, in the small angle approximation $\left(\mathrm{q}<\mathrm{q}^{*}\right)$ :

$$
\left\langle n_{\alpha}\right\rangle k T \chi_{T}=\sum_{\beta} v_{\beta} S_{\alpha \beta}(0) \quad \text { for } \mathrm{q}<\mathrm{q}^{*}
$$

By combining equations (15) and (17), it is possible to remove one of the elementary scatter, for instance $\alpha=1$ (which it is generally a continuous phase such as the matrix or the solvent) by introducing the contrast length $\mathbf{k}_{\mathbf{a}}=b_{\alpha}-b_{1} \frac{v_{\alpha}}{v_{1}}$ with respect to the ES $\alpha=1$ in order to obtain the general equation of coherent scattering in SANS:

$$
\begin{array}{r}
\Sigma(q)=\sum_{\alpha, \beta=2}^{m} k_{\alpha} k_{\beta} S_{\alpha \beta}(q)+\frac{b_{1}}{v_{1}} \sum_{\alpha=1}^{m}\left\langle n_{\alpha}\right\rangle\left(k_{\alpha}+b_{\alpha}\right) k T \chi_{T} \\
\text { for } \mathrm{q}<\mathrm{q}^{*}
\end{array}
$$

The second term of this equation is related to the compressibility of the system weighted by the respective contrasts of the different scatterers. For $\mathrm{q}<\mathrm{q}^{*}$, it is $\mathrm{q}$-independent and will be considered as a flat background that has to be subtracted from the experimental scattered intensity, as for the incoherent scattering (see part 3.3.3). It is generally negligible compared to the other contributions to the scattering and is therefore often omitted, as we will do in the following.

Let us now consider the simplest possible case of a system made only of two types of elementary scatterers. This is the common case, as it describes objects (nanoparticles, polymers, colloids ...) in a continuous media (matrix, solvent, continuous phase...). Focusing on the first part of the equation, it comes that:

$$
\Sigma(q)=\left(b_{2}-b_{1} \frac{v_{2}}{v_{1}}\right)^{2} S_{22}(q)
$$

Or, if considering the scattering length densities of the components:

$$
\Sigma(q)=\left(\rho_{2}-\rho_{1}\right)^{2} v_{2}^{2} S_{22}(q)=(\Delta \rho)^{2} v_{2}^{2} S_{22}(q)
$$

The contrast term $\left(\rho_{2}-\rho_{1}\right)^{2}$ is thus a prefactor of the scattered intensity. Tuning this prefactor by changing the SLD of the continuous can either strongly enhance such intensity or in the reverse "kill" it since it is linear to the square of the difference between the SLD of the components. This is the basis of the contrast variation experiments which will be described in more details in part 2.7. 

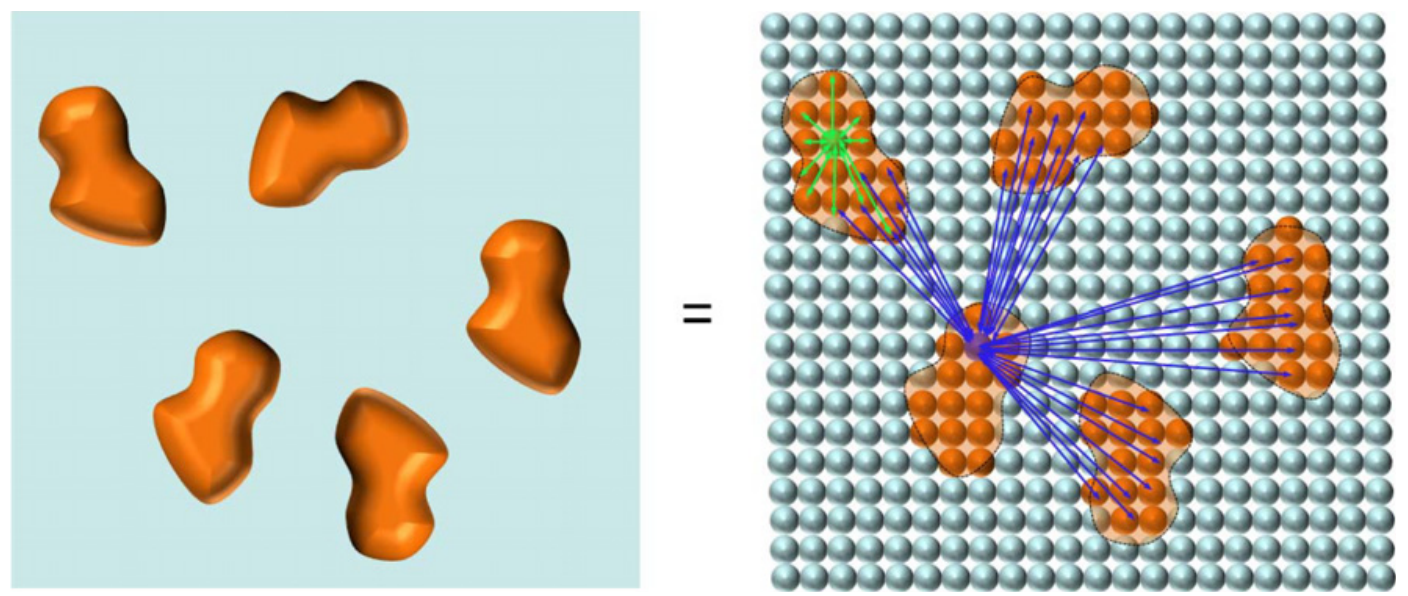

Figure 4. Form factor and structure factor. The form factor comes from interferences between elementary scatterers from the same object (see green arrows in right panel) while the structure factor comes from interferences from elementary scatterers belonging to different objects (blue arrows in right panel).

\subsection{Form factor and structure factor}

\subsubsection{General case}

Let us now consider a binary system of $n$ similar objects containing $\mathrm{N}$ elementary scatterers in a continuous media. Following Eq. (20), there is only one partial structure factor $\mathrm{S}_{o b j \_o b j}(\mathrm{q})$ and the scattered intensity writes $\Sigma(q)=\left(\rho_{o b j}-\rho_{\text {media }}\right)^{2} v_{o b j}{ }^{2} S_{o b j \_o b j}(q)$ where $\rho_{\text {obj }}$ and $\rho_{\text {solv }}$ are respectively the SLD of the object and of the solvent and $v_{\text {obj }}$ is the volume of an elementary scatterer of the object.

It is useful to separate in $S_{\text {obj_obj }}(q)$ the respective contributions to the scattering arising from interferences from elementary scatterers belonging to the same object or from interferences arising from elementary scatterers belonging to different objects:

$$
\frac{\Sigma(q)}{\left(\rho_{\text {obj }}-\rho_{\text {media }}\right)^{2} v_{o b j}{ }^{2}}=\sum_{\alpha}^{n} \sum_{\beta}^{n} \sum_{i}^{N} \sum_{j}^{N} e^{i \vec{q} \cdot\left(\vec{r}_{i}^{\alpha}-\vec{r}_{j}^{\beta}\right)}
$$

There are thus $(\mathrm{nN})^{2}$ parts in the scattered intensity, with $\mathrm{n}^{2} \mathrm{~N}$ coming from intra-objects interferences and $(\mathrm{n}-1) \mathrm{nN}^{2}\left(\approx \mathrm{n}^{2} \mathrm{~N}^{2}\right)$ from inter-objects interferences (see Fig. 4$)$ :

$$
\frac{\Sigma(q)}{\left(\rho_{o b j}-\rho_{\text {media }}\right)^{2} v_{o b j}{ }^{2}}=n \sum_{i, j}^{N} e^{i \vec{q} \cdot\left(\vec{r}_{i}^{\alpha}-\vec{r}_{j}^{\alpha}\right)}+n^{2} \sum_{i, j, \alpha \neq \beta}^{N} e^{i \vec{q} \cdot\left(\vec{r}_{i}^{\alpha}-\vec{r}_{j}^{\beta}\right)}
$$

The following normalized physical quantities are classically introduced:

$$
\begin{aligned}
& P(q)=\frac{1}{N^{2}} \sum_{i^{\alpha}}^{N} \sum_{j^{\alpha}}^{N} e^{i \vec{q} \cdot\left(\vec{r}_{i}^{\alpha}-\vec{r}_{j}^{\alpha}\right)} \\
& Q(q)=\frac{1}{N^{2}} \sum_{i^{\alpha}}^{N} \sum_{j^{\beta}}^{N} e^{i \vec{q} \cdot\left(\vec{r}_{i}^{\alpha}-\vec{r}_{j}^{\beta}\right)}
\end{aligned}
$$


Equation (22) then writes:

$$
\frac{\Sigma(q)}{n N^{2}\left(\rho_{o b j}-\rho_{\text {media }}\right)^{2} v_{o b j}{ }^{2}}=(P(q)+n Q(q))
$$

The intra term $\mathbf{P}(\mathbf{q})$ is the form factor of the object that contains the information on the correlations between its own elementary scatterers and the inter term $\mathbf{Q}(\mathbf{q})$ is the structure factor that contains the information on the correlation between elementary scatterers from different objects (Fig. 4).

\subsubsection{Case of rigid centro-symmetrical objects}

Let us now consider the case where the objects are rigid and centro-symmetrical. In this case, and only in this case, the amplitudes and form factors are independent from the orientation and positions of objects and the intra and inter can be decoupled (see (12) by choosing the origin of the objects in their center of mass. Let us consider the respective centers of mass $\alpha$ and $\beta$ of objects: $\overrightarrow{i j}=(\overrightarrow{i \alpha}+\overrightarrow{\beta j})+\overrightarrow{\alpha \beta}$. Since they are centro-symmetrical, there is for every point $j$ of an object of center of mass $\beta$ a point $j^{\prime}$ of the object of center of mass $\alpha$ such as $\overrightarrow{\beta j}=\overrightarrow{\alpha j^{\prime}}$, and then $(\overrightarrow{i \alpha}+\overrightarrow{\beta j})=\overrightarrow{i j^{\prime}}$ is independent from $\alpha$ and $\beta$. It is then possible to make a factorization of the sums in Eq. (22) $\left(\sum_{\propto}^{n} \sum_{\beta}^{n} \sum_{i}^{n} \sum_{j}^{n} e^{i \vec{q} \cdot\left(\vec{r}_{i}^{\alpha}-\vec{r}_{j}^{\beta}\right)}=\right.$ $\left.\sum_{\alpha}^{n} \sum_{\beta}^{n} e^{i \vec{q} \cdot \overrightarrow{-\alpha \beta}} \times \sum_{i}^{N} \sum_{j,}^{N} e^{i \overrightarrow{q-i j^{\prime}}}\right)$.

The scattering intensity writes then:

$$
\frac{\Sigma(q)}{n N^{2}\left(\rho_{o b j}-\rho_{\text {media }}\right)^{2} v_{o b j}{ }^{2}}=P(q) \times S(q)
$$

With

$$
\begin{aligned}
& P(q)=\frac{1}{N^{2}} \sum_{i}^{N} \sum_{j^{\prime}}^{N} e^{i \vec{q} \cdot \overrightarrow{i j j^{\prime}}} \\
& P(q)=\frac{1}{N^{2}} \sum_{\alpha}^{N} \sum_{\beta}^{N} e^{i \vec{q} \cdot \overrightarrow{\alpha \beta}}
\end{aligned}
$$

where $\mathbf{S}(\mathbf{q})$ is the structure factor that describes the correlations between the centers of mass of the objects (Fig. 5). Note that the scattering intensity is the product (Eq. (26)) of the form factor and of the structure factor for centro-symmetric scattering objects while it is the sum (Eq. (25)) in the general case. In soft matter, this is the difference between colloids and polymers.

\subsection{What can be extracted from the analysis of the form factor}

In this section, we consider the case of a binary system of $\mathrm{n}$ objects in a continuous media without interobjects correlations. This situation arises in very dilute systems, the system behaving then like a perfect gas, or when interactions between objects are negligible. The scattered intensity reduces then to the form factor of the object (see previous part). This scattered intensity enables a very fine characterization of the shape and volume of the objects as several information can be obtained depending on the q-range which is probed: (i) the volume of the object (and thereby its mass) for $\mathrm{I}(0)$, (ii) its gyration radius in the Guinier regime at low q, (iii) the shape and dimensions in the intermediate regime at intermediate $\mathrm{q}$ and finally (iv) its specific area S/V a large $\mathrm{q}$ in the Porod regime (see Fig. 6). These different regimes will be detailed here. 

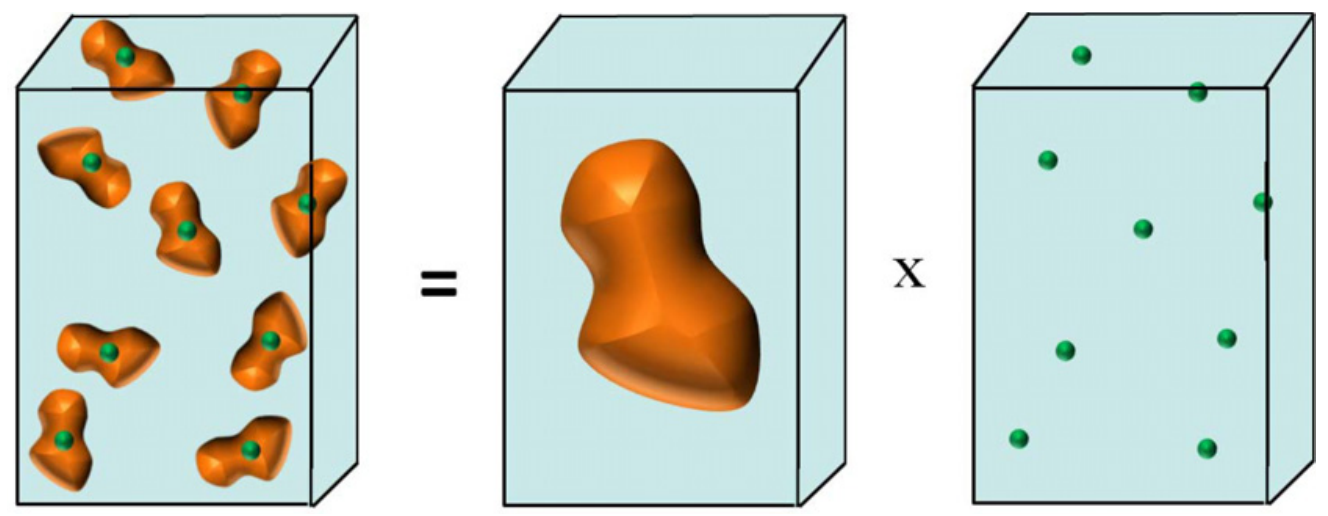

Figure 5. Form factor and structure factor for rigid centro-symmetrical objects. The scattered intensity is the product of the form factor to the structure factor.

We will focus on the case where the system is isotropic and that all objects are free to rotate around their mass center. The form factor is then an average over all possible orientations. It may then be demonstrated that it reduces to:

$$
P(q)=\frac{1}{N^{2}} \sum_{i}^{N} \sum_{j}^{N}\left\langle e^{i \vec{q} \cdot \overrightarrow{r_{i j}}}\right\rangle=\frac{1}{N^{2}} \sum_{i}^{N} \sum_{j}^{N} \frac{\sin \left(q r_{i j}\right)}{q r_{i j}} .
$$

\subsubsection{Volume/Mass of the object}

When $\mathrm{q} \rightarrow 0, \mathrm{P}(\mathrm{q}) \rightarrow 1 . I(q)$ reduces thus to:

$$
I(q)=\left(\rho_{o b j}-\rho_{\text {media }}\right)^{2} v_{o b j}{ }^{2} n N^{2}=\left(\rho_{o b j}-\rho_{\text {media }}\right)^{2} n V_{o b j}
$$

where $\mathrm{V}_{\text {obj }}$ is the volume of the object.

Indeed, the scattered intensity of a single object $\mathrm{I}_{\mathrm{obj}}(0)$ equals the square of the total number of its constitutive elementary scatterers because, at $\mathrm{q}=0$, all the scattered waves are in phase and the amplitudes are added.

The determination of the $I(q)_{q \rightarrow 0}$ enables thus the determination of the volume of the object, whatever its shape and without any modeling.

In practice, the experimentalist often knows the volume fraction of the objects in its sample $\Phi_{\text {obj }}$ $\left(\mathrm{nV}_{\mathrm{obj}}\right)$, then:

$$
I(q=0)=\left(\rho_{o b j}-\rho_{\text {media }}\right)^{2} \Phi_{o b j} V_{o b j}
$$

$\mathrm{I}(\mathrm{q})_{\mathrm{q} \rightarrow 0}$ is often also used to determine the molar mass $\mathrm{M}_{\mathrm{w}}$ (in $\mathrm{g} / \mathrm{mol}$ ) of a macromolecule in solution, knowing its concentration $\mathrm{c}(\mathrm{g} / \mathrm{L})$ and the molar mass $(\mathrm{g} / \mathrm{mol})$ of the elementary scatterer constitutive of the macromolecule, which is usually a repetition unit in case of a polymer chain.

\subsubsection{Guinier regime}

The Guinier regime refers to the low q-regime of the scattering curve for which one probes spatial scales large enough so that $\mathrm{q}^{-1}$ is much larger that the size of the objects. It is then possible to make a limited development of the sine function $\left(\sin x=x-x^{3} / 3 !+o x^{5}\right)$ in Eq. (28) since all $\mathrm{qr}_{i j} \ll 1$ : 


\section{JDN 21}

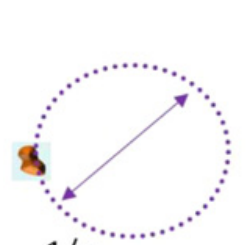

$1 / \mathrm{q}$
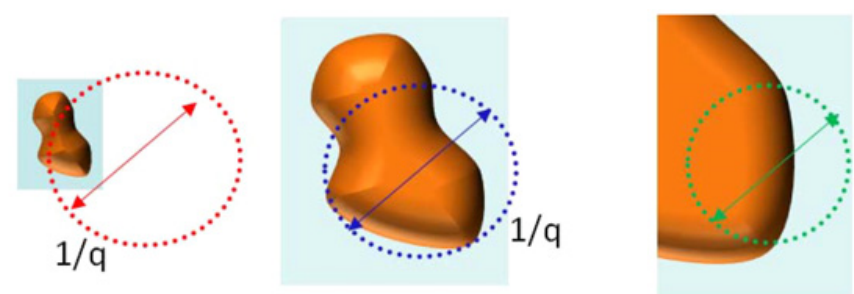

$1 / q$

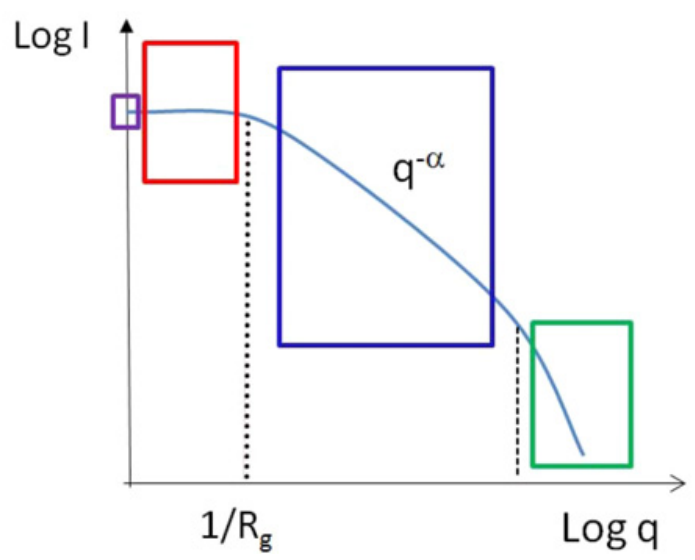

Figure 6. Scattered intensity and form factor. The upper panels show the typical size $\mathrm{q}^{-1}$ probed by the neutrons with respect to the object size for the different regimes. From left to right: I(q) $)_{q \rightarrow 0}$ : volume object (or mass); Guinier regime: gyration radius; Intermediate regime: shape and dimensions of object; Porod regime: specific surface S/V of the sample.

$$
P_{\text {Guinier }}(q)=1-\frac{q^{2}}{3}\left[\frac{1}{2 N^{2}} \sum_{i}^{N} \sum_{j}^{N} r_{i j}^{2}\right]=1-\frac{q^{2} R_{g}^{2}}{3}
$$

with $R_{g}^{2}=\frac{1}{2 N^{2}} \sum_{i}^{N} \sum_{j}^{N} r_{i j}^{2}$ where $\boldsymbol{R}_{\boldsymbol{g}}$ is the gyration radius of the object. It is more convenient to express it considering the distances from the gravity center of the object $\left(\sum_{i}^{N} \vec{r}_{i}=0\right)(12)$. It comes then:

$$
R_{g}^{2}=\frac{1}{N} \sum_{i}^{N} r_{i}^{2}=\left\langle r_{i}^{2}\right\rangle
$$

This result is valid whatever the shape of the object, as long as $q R_{g}<1$, in the so-called Guinier regime.

The gyration radius is thus the size associated to the moment of inertia of the object $=m R_{g}^{2}$. It indeed corresponds to the radius of the empty sphere that would have the same moment of inertia as the object.

For a sphere, the gyration radius is lower than its radius $\mathrm{R}$ since some elementary scatterers are not located at the outer edge of it. Replacing the sum in Eq. (32) by an integral enables a fast calculation of $R_{g}^{2}=\frac{\int_{0}^{R} 4 \pi r^{2} \times r^{2} \times d r}{\int_{0}^{R} 4 \pi r^{2} \times d r}=\frac{3}{5} R^{2}$.

For a cylinder of length $\mathrm{L}$ and of radius $\mathrm{R}$, one gets $R_{g}^{2}=\frac{R^{2}}{2}+\frac{L^{2}}{12}(5)$. An interested reader may find several gyration radii calculations for different rigid objects of various geometries (5). 
The measurement of the gyration radius is very important in polymer physics as it is the most relevant quantity to characterize its size, given that it is proportional to the root mean square distance between the monomers. In this case, it has to be kept in mind that the measurement is averaged over time over all possible configurations. For a Gaussian chain, id est a chain without interaction, the conformation of the chain is a random walk and $R_{g}=a / \sqrt{6} N^{1 / 2}$ where a is the size of a repetition unit (the Kuhn length) and $\mathrm{N}$ the number of repetition units. For a chain in good solvent with excluded volume interactions, $R_{g} \simeq a N^{0.577}$.

Measuring the gyration radius is in practice very easy. At very low $q$ in the Guinier regime, when $q R_{g} \ll 1$, Eq. (31) is generally approximated by:

$$
P_{\text {Guinier }}(q) \approx e^{-\frac{q^{2} R_{g}^{2}}{3}}
$$

The experimental data have thus to be plotted in the In $(I(q))$ versus $q^{2}$ representation, the so-called Guinier representation, that gives a linear decay whose slope is $\frac{R_{g}^{2}}{3} R_{g}$, so a direct measurement of $R_{g}$ in the limit $\mathrm{qRg} \sim<1$.

\subsubsection{Intermediate regime}

The intermediate regime is the q-regime for which the richest information can be extracted, since it enables the determination of the shape and size of the object. In general, the scattered intensity presents an overall $I(q) \sim q^{-\alpha}$ decay where $\alpha$ is related to the shape of the object. For bulky objects, $\alpha$ is 4. With hands, it comes from the fact that one probes the intersection of the object $\left(\propto\left(\frac{1}{q}\right)^{3}\right)$ with the neutron $\left(\frac{1}{q}\right)$. For objects that have a fractal dimension $D_{\mathbf{f}}, \boldsymbol{d}$ gives directly the fractal dimension of the object $\alpha=\mathbf{D}_{\mathbf{f}}$ (see later), id est a 1-D object will have a scattering decay $\mathrm{q}^{-1}$ like and a 2 -D object a scattering decay like $\mathrm{q}^{-2}$. Depending on the probed q-range, there could be a crossover between two qdecay regimes whether one probes large sizes (low q's) or local size (large q's). For instance, an infinite cylinder of finite radius scatters like $\mathrm{q}^{-1}$ at small $\mathrm{q}$ as the cylinder appears like an infinite rod to the neutron and like $\mathrm{q}^{-4}$ at large $\mathrm{q}$ as the cylinder appears here like a bulky object. Along the same lines, in polymer physics, a wormlike chain scatters like $\mathrm{q}^{-2}$ at small $\mathrm{q}$ if in theta solvent (Gaussian behavior) or like $\mathrm{q}^{-1.7}$ if in good solvent but locally like $\mathrm{q}^{-1}$ since the chain resembles a rod. The cross-over between the two regimes is used to extract the persistence length without any fitting (13).

Analytical form factors

When an object has a well-defined geometry (bulk sphere, empty sphere, cylinder, discs, ellipsoid, paralleliped, etc...), its form factor has an exact analytical form. This adds specific features to the scattered intensity to the overall scattering decay depicted here before, such as maxima or extinctions at given q's occurring from constructive or fully destructives interferences ${ }^{\dagger}$. The q-positions extinctions are very useful to determine the size of the objects because they enable a fast evaluations of the size of the object to probe without any fitting (see the example for spheres in par 2.5.8).

For the calculation of these analytical form factors, it is convenient to describe the object by its density function $\rho(\mathrm{r})(\rho(\mathrm{r})=1$, if an elementary scatterer belong to the object and $\rho(\mathrm{r})=0$ if not) and to consider an integral rather than a sum in Eq. (28).

For an empty sphere of radius $\mathrm{R}$ with an infinite thin thickness, the calculation of trivial $(\rho(\mathrm{r})=1$ only for $\mathrm{r}=\mathrm{R}$ ). The form factor then writes:

$$
P_{\text {empty sphere }}(q)=\left[\frac{\sin (q R)}{q R}\right]^{2}
$$

\footnotetext{
${ }^{\dagger}$ It is by principle analogous to the minima of the Airy function describing the scattering by a circular hole.
} 
Table 1. Some analytical form factor of common objects. ${ }^{*}$ refers to calculations form factors averaged over all directions. $j_{0}(x)$ is the zero order Bessel function $(\sin (x) / x)$ and $j_{1}(x)$ is the first order Bessel function $\left(\sin (x) / x^{2}-\right.$ $\cos (x) / x)$.

\begin{tabular}{|c|c|}
\hline $\begin{array}{l}\text { Spherical shell of outer } \\
\text { radius } R_{2} \text { and inner } \\
\text { radius } R_{1}\end{array}$ & $P_{\text {spherical shell }}(q)=\left[\frac{1}{R_{2}{ }^{3}-R_{1}{ }^{3}}\left[\left(\frac{3 j_{1}\left(q R_{2}\right)}{q R_{2}}\right) R_{2}{ }^{3}\left(\frac{3 j_{1}\left(q R_{1}\right)}{q R_{12}}\right) R_{1}{ }^{3}\right]\right.$ \\
\hline $\begin{array}{l}\text { Rod of length } \mathrm{L} \text { and } \\
\text { negligible radius* }\end{array}$ & $\begin{array}{l}P_{\text {rod }}(q)=\frac{2}{q L} \int_{0}^{q L / 2}\left(j_{0}(y)\right)^{2} d y \text { with } y=q L \cos \phi / 2 \\
\quad \text { Note that } P_{\text {rod }}(q L \gg 1)=\frac{\pi}{q L}\end{array}$ \\
\hline $\begin{array}{l}\text { Disk of radius } \mathrm{R} \text { and } \\
\text { negligible thickness* }\end{array}$ & $P_{\text {disk }}(q)=\frac{2}{(q R)^{2}}\left[1-\frac{j_{1}(2 q R)}{(q R)^{2}}\right]$ \\
\hline $\begin{array}{l}\text { Rectangular parallelepiped } \\
\text { of thickness a, width } b \text {, and } \\
\text { length } c^{*}\end{array}$ & $\begin{array}{l}P_{\text {paralleliped }}(q)= \\
\frac{2}{\pi} \int_{0}^{\frac{\pi}{2}} \int_{0}^{\frac{\pi}{2}} \frac{\sin (q a \sin \alpha \cos \beta)}{q a \sin \alpha \cos \beta} \frac{\sin (q b \sin \alpha \sin \beta)}{q b \sin \alpha \sin \beta} \frac{\sin (q \cos \alpha)}{q \cos \alpha} \sin \alpha d \alpha d \beta\end{array}$ \\
\hline Gaussian chain & $P_{\text {gaussian chain }}(q)=\frac{2}{\left(q^{2} R^{2}\right)^{2}}\left(q^{2} R^{2}-1+e^{-q^{2} R^{2}}\right)$ with $R=R_{g}$ \\
\hline
\end{tabular}

The overall decay is then like $\mathrm{q}^{-2}$ since one probes an object reduced to its surface.

For a bulk sphere, the calculation of the form factor is simple due to the spherical geometry:

$$
P_{\text {sphere }}(q)=\left[\int_{0}^{R} 4 \pi r^{2} \frac{\sin (q r)}{q r} d r\right]^{2}
$$

That gives, after an integration by parts:

$$
P_{\text {sphere }}(q)=\left[3 \frac{\sin (q R)-q R \cos (q R)}{(q R)^{3}}\right]^{2}
$$

The overall decay of such a form factor is here like $\mathrm{q}^{-4}$.

Table 1 presents several form factors of other common objects (12) (5). Some other form factors (ellipsoid, twisted ribbons, etc... ) can be found in (5). Various form factors are generally computed in the analysis programs of Small Angle Scattering, the two most famous being SASView (14) and SASfit (15), free to download.

Self-similar objects and fractal dimension

When an object has not a well-defined shape, its fractal dimension $\mathrm{D}_{\mathrm{f}}$ can be directly obtained from the overall decay of the scattering in the intermediate regime. The fractal dimension links the mass of an object $\mathrm{M}$ to its characteristics size $\mathrm{R}$ through:

$$
M(R) \propto R^{D_{f}}
$$

The fractal dimension enables to characterize self-similar objects, id est objects that have the same geometrical shape whatever the magnification they are looked. This fractal dimension has not necessary an integer value like for Euclidean object.

In the experimental range for which the object has a fractal behavior, the density distribution is $g(r) \propto r^{D_{f^{-d}}}$ in a $d$-dimensions space (16). And thus, by Fourier transform:

$$
P(q) \propto \frac{1}{q^{D_{f}}}
$$


It is thus very easy to extract the fractal dimension of a system by a scattering experiment as it is a simple reading of the slope value in $\log -\log$ representation of $\mathrm{I}(q)$ versus $q$. For the fractal nature of the system behavior to be meaningful, it has however to be observed on a sufficiently large q-range, one decade at least. The fractal dimension is very useful to characterize aggregates (see part 2.6.5).

Exact form factor of an object with an irregular shape

When an object has an irregular shape, it is also possible to extract its exact form with a 'low resolution' (by comparison to what can be obtained from diffraction) by a numerical method based on simulated annealing. This technique, popularized by Svergun (17), is generally used to make the calculation of the form factor of proteins or aggregates. The principle is as follows: one first built an object with an assembly of elementary scatterers whose sizes are typically of the order of $1 / q_{\max }$ where $\mathrm{q}_{\max }$ is the maximal experimental scattering vector probed during the experiment. In case of proteins, each elementary scatterer may have a given SLD in order to take into account the difference in chemical composition from one residue to another. The form factor of the object is then calculated according to Eq. (28). The shape of the object is then progressively changed iteratively by moving some elementary scatterers in order to minimize numerically the $\chi^{2}$ between experimental and theoretical spectra. The minimization is achieved by a Monte Carlo technique. This step may be tricky and it is a good prerequisite to have a general idea of the shape of the object prior to the experiment to build a primary object which is not far from the final solution, for instance with help of a classical SANS analysis. Also some constraints on quantities such are connectivity or compacity can be imposed during the minimization step. From experimental point of view, it is important to measure in dilute regime to ensure that the experimental spectrum is not sensitive to the structure factor.

\subsubsection{Cut function at large $q$}

In previous part, the form factors were obtained in q-range for which the elementary scatterer was considered as punctual. For instance, the radius of the rod was neglected in the formula of the form factor of the rod presented in Table 1, as well as the thickness of the disc in the formula of the form factor of the disc. However, at large q, this approximation is no longer valid and the measurement becomes sensitive to the structure of the elementary scatterer at local scale. When this local structure has a spherical symmetry, it is possible to decouple the respective contributions of the overall form factor $P_{\text {overall }}(\mathrm{q})$ and the form factor at the local scale $P_{\text {local }}(q)$, similarly to what has been done in Eq. (26) to separate the form factor and the structure factor (12):

$$
P(q)=P_{\text {overall }}(q) \times P_{\text {local }}(q)
$$

$P_{\text {overall }}(\mathrm{q})$ is here form factor of the object considering the elementary scatterers as punctual and reduced to their gravity center and $P_{\text {local }}(q)$ is the form factor of the elementary scatterers.

If the observation scale $\mathrm{q}^{-1}$ is larger than the size $a$ of the elementary scatterer, it is possible to approximate $\mathrm{P}_{\text {local }}(\mathrm{q})$ by the Guinier function and Eq. (39) becomes:

$$
P(q)=P_{\text {overall }}(q) \times e^{-\frac{q^{2} a^{2}}{3}}
$$

This last equation is very useful to extract precisely the thickness of the wall $a$ in an empty sphere with a very large radius such as a large vesicle or the droplet of an emulsion which are infinite planes, scattering like $\mathrm{q}^{-2}$, in the window of observation of neutrons. As this is based on a Guinier approximation, $a$ can be obtained without any fitting. 


\subsubsection{Porod regime/Scattering Invariant}

The Porod regime refers to the regime where only the local scales are probed, id est $\mathrm{q}^{-1}$ is much smaller than the size of the objects. At such scales, the scattering intensity is only sensitive to the interfaces between two homogeneous media with different SLD. Indeed, only the parts of the sample located in the vicinity of the interface scatter because all of the other parts do not scatter since they are homogeneous from the point of view of SLD $\ddagger$. In a two-components system of respective SLD $\rho_{1}$ and $\rho_{2}$, if the interfaces between the two components is perfectly smooth at the $\mathrm{q}^{-1}$ scale, the scattered intensity varies like $\mathrm{q}^{-4}$ following the Porod law that is proportional to the surface area per unit volume $\mathbf{S} / \mathbf{V}$ of the sample, id est the quantity of scattering surfaces by unit volume:

$$
I(q)=\frac{2 \pi\left(\rho_{2}-\rho_{1}\right)^{2}}{q^{4}} \frac{S}{V}
$$

Since, one probes only interfaces, the Porod law may be understood with hands in the framework of reflectivity. Indeed, the scattered intensity only results from the reflections of the incident beam on the parts of the sample which have the correct orientation to provide a reflected beam towards a given angle of scattering. It is then similar to the Fresnel reflectivity that decays like $\mathrm{q}^{-4}$ (see the reflectivity course in this volume). The demonstration of the Porod law can be found in ref. (18).

The Porod law is valid whatever the geometry for any kind of a two-phase system. For isolated objects the aim of the experimentalist is generally to obtain the surface to volume for the object itself $S_{o b j} / V_{o b j}$ and then the Porod law has to be rewritten taking into account the volume fraction $\Phi_{o b j}$ with $S / V=\Phi_{o b j}\left(1-\Phi_{o b j}\right) S_{o b j} / v_{o b j}$. For monodisperse spheres, the Porod radius $\mathrm{R}_{P}$ can be estimated like: $3 / R_{p}=S_{o b j} / v_{o b j}$. Note that the determination of $\mathrm{S} / \mathrm{V}$ requires scattering intensity in absolute units.

When the interface is no longer smooth but rough or diffuse, or when the two media are interpenetrated, this $\mathrm{q}^{-4}$ scattering may be multiplied by the Fourier transform of the SLD profile of the interface. The analysis of the scattering may enable to determine such a profile. Further deepening and examples on these variations around the Porod law can be found in ref. (18).

It is also possible to extract the respective volume fraction of phases in a incompressible two-phase system thanks to the invariant scattering $Q$. Indeed, Porod showed that the total small angle scattering from a sample is a constant irrespective of the way the sample density is distributed (3).

The scattered intensity can thus be integrated with respect to q:

$$
Q=\int_{v} \Sigma(\vec{q}) d_{3} \vec{q}=4 \pi \int_{0}^{\infty} I(q) q^{2} d q=8 \pi^{3} \Phi_{1}\left(1-\Phi_{1}\right)\left(\rho_{2}-\rho_{1}\right)^{2}
$$

\subsubsection{The determination of the scattering invariant requires a measurement of the scattered intensity over a very large q-range. Since in practice experiments are often performed on a limited q-range, the scattering invariant is seldom measured, although it is very powerful method to value the volume fraction, or concentration, of objects. Representations}

As any form factor $P(q)$ has generally a very fast decay when going towards large q, its common representation (and therefore the one of the scattered intensity) is usually the $\log (I(q)) v \operatorname{ersus} \log (q)$ representation. However, depending on the regimes probed, some other useful representations such as the Guinier representation $\ln (\boldsymbol{I}(\boldsymbol{q}))$ versus $\boldsymbol{q}^{2}$, that gives a slope proportional to $R_{g}$ or or the Porod representation $I(q) q^{4}$ versus $q$ that gives a constant proportional to S/V. Also, when form factor decays like $\mathrm{q}^{-\alpha}, \mathrm{t}$ is very convenient to use a $\boldsymbol{I}(\boldsymbol{q}) \boldsymbol{q}^{\alpha}$ versus $\boldsymbol{q}$ representation that makes appear a constant

\footnotetext{
$\ddagger$ A homogeneous media from the point of view of SLD does not scatter because, in average, there is always a wave scattered by an elementary scatterer which is phase opposite to a wave scattered by an other elementary scatterer. The sum of all spherical waves scattered permanently by the scatterers give rise back to a planar wave.
} 
and enables to show deviations or details from the decay law. The most commonly used is the Kratky representation $I(q) q^{2}$ versus $q$ which is very useful for the study of polymers.

\subsubsection{Polydisperse systems}

The objects probed by SANS are seldom monodisperse systems. Their radius $\mathrm{R}$ in case of spheres or chain length $\mathrm{N}$ in case of polymeric chain usually follow a polydispersity distribution law $f(R)$ (with $\left.\int_{0}^{\infty} f(r) d R=1\right)$. The most common are the Gaussian distribution, the lognormal distribution, which is often encountered in colloidal system made of inorganic nanoparticles or the Schulz distribution that describes the molecular weight distribution of synthetic polymers (see chapter 29 of (5)). The relationship between the gyration ratio and the mean radius are linked to some of the $\mathrm{n}^{\text {th }}$ moment of the radius $\left\langle R^{n}\right\rangle=\int_{0}^{\infty} R^{n} f(R) d R$.

When the density number of objects $n / \mathrm{V}$ is independent from polydispersity, then:

$$
\left\langle R_{g}{ }^{2}\right\rangle=\frac{3}{5} \frac{\left\langle R_{8}\right\rangle}{\left\langle R_{6}\right\rangle}
$$

Or when the volume fraction is independent from polydispersity:

$$
\left\langle R_{g}{ }^{2}\right\rangle=\frac{3}{5} \frac{\left\langle R_{5}\right\rangle}{\left\langle R_{3}\right\rangle}
$$

The exact values of $\left\langle R_{3}\right\rangle,\left\langle R_{5}\right\rangle,\left\langle R_{6}\right\rangle$ and $\left\langle R_{8}\right\rangle$, for the three distributions Gaussian, lognormal and Schulz distributions can be found in (5).

\subsubsection{Experimental example: System of diluted nanospheres in aqueous media}

We show here as an example the archetypical case of a system of spheres in diluted regime. The system is made of negatively charged silica spheres of nanometric size in an aqueous solvent (normal water here). In order to make the repulsive negative interactions between nanoparticules negligible, the mother stock solution spheres has been diluted down to $\Phi_{\text {sil }}=0.012$, the $\mathrm{pH}$ has been set to $\sim 7$ and the ionic strength to $\mathrm{I}=0.01 \mathrm{M}^{\S}$. Figure 7 a shows the scattered intensity $\mathrm{I}(\mathrm{q})$ in $\log$-log representation, after data reduction and subtraction of incoherent scattering (see part 3). At first sight, the global decay of the scattering varies like $\mathrm{q}^{-4}$ showing that one probes a bulky object at intermediate $\mathrm{q}$ and a Guinier plateau at low q, showing the spheres are small enough to be fully characterized within the probed q-range. Looking down to Eq. (36) (form factor of sphere) it appears that there are extinctions at some specifics q's, when $\tan (\mathrm{qR})=\mathrm{qR}$, thus for $\mathrm{q}_{1} \mathrm{R}=4.49, \mathrm{q}_{2} \mathrm{R}=7.72, \mathrm{q}_{2} \mathrm{R}=10.91$, etc.). Experimentally, there is an oscillation corresponding to the first extinction at $\mathrm{q}_{1} \sim 0.055 \AA^{-1}$. This gives a raw estimate of the radius of spheres $\mathrm{R}_{\text {sil }}$ of $4.49 / 0.55 \sim 80 \AA$. The second minima should be then located at $\mathrm{q}_{2} \sim 7.72 / 80=0.096 \AA^{-1}$. This second minima is hardly visible on Fig. 7a due to statistics. Please note that it is much easy to observe the extinctions in the Porod representation (Fig. 7c) since the multiplication by $\mathrm{q}^{4}$ enables to compensate the overall $\mathrm{q}^{-4}$ behavior decay in order to probe the detailed features of the scattering curve, similarly as a Fresnel representation in a neutron reflectivity experiment.

The intensity $\mathrm{I}(\mathrm{q})_{q \rightarrow 0}$ is around $39 \mathrm{~cm}^{-1}$ and also enables to evaluate the radius. The SLD of silica is $3.4110^{10} \mathrm{~cm}^{-2}$ and those of $\mathrm{H}_{2} \mathrm{O}$ is $-0.5610^{10} \mathrm{~cm}^{-2}$. Thus, according to equation, $\mathrm{V}_{\text {sil }}$ is $\mathrm{I}(\mathrm{q} \rightarrow 0) / \Delta \rho^{2} \Phi_{\text {sil }}=39 /\left(0.012 *\left(3.4110^{10}--0.5610^{10}\right)^{2}\right) \sim 2.0610^{-18} \mathrm{~cm}^{3}$, or $2.0610^{6} \AA^{3}$. This gives $\mathrm{R}_{\text {sil }} \sim 79 \AA$, in perfect accordance with the value derived from the estimate from the extinction!

\footnotetext{
$\S$ Such system is perfect for a half-day SAS practice, whether it is SANS or SAXS. Mother concentrated stock solutions of almost monodisperse nanospheres of various mean radii can be purchased indeed at very low prices. $\Phi_{\text {sil }}$ is very easy to estimate by gravimetry.
} 

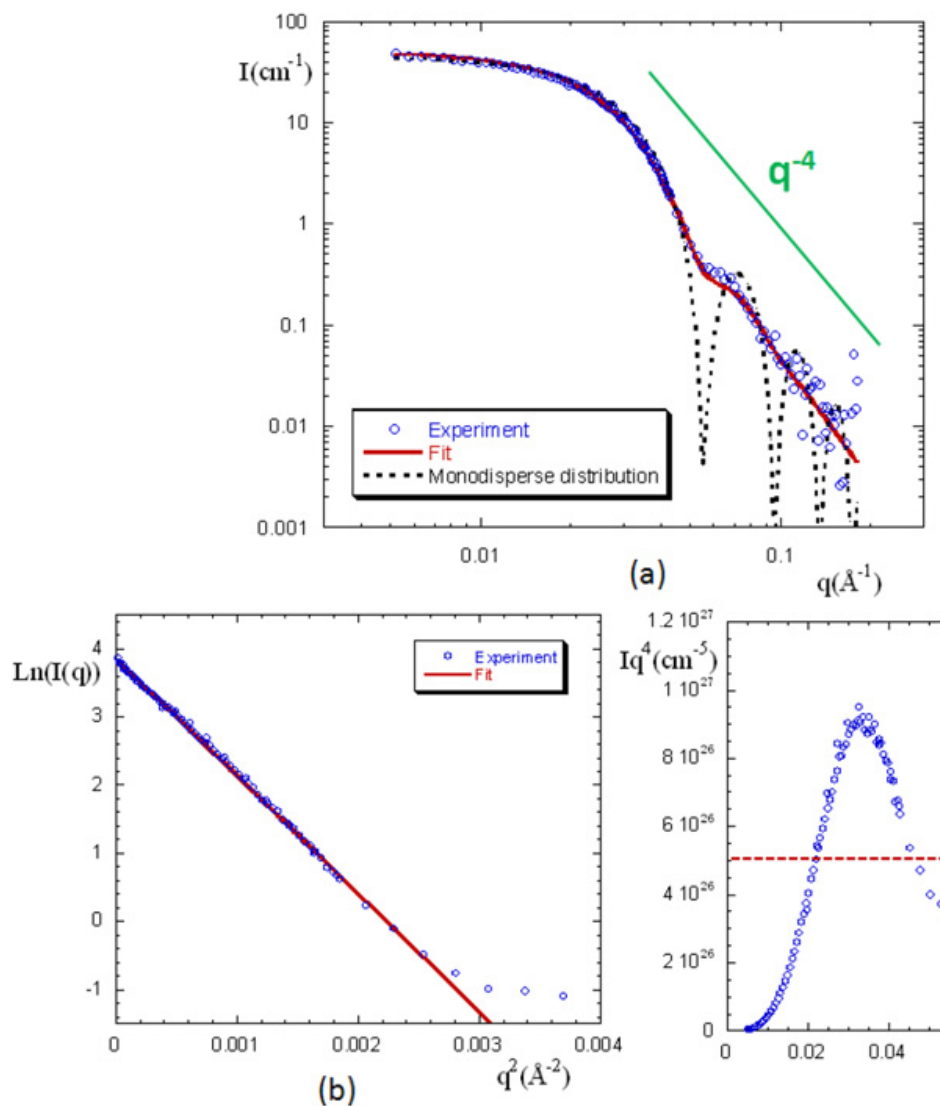

(a) $\quad \mathrm{q}\left(\AA^{-1}\right)$

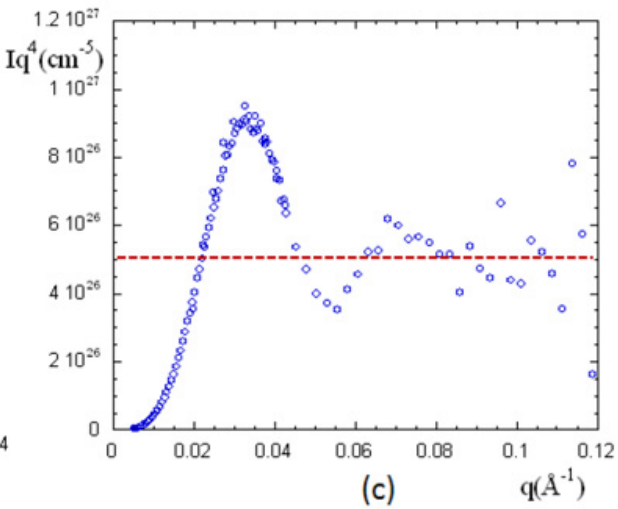

Figure 7. Scattering from a diluted suspension of silica nanospheres in water. (a) Log-log representation; (b) Guinier representation; (c) Porod representation.

In order to go further in the modeling, the dotted line of Fig. 7a shows the calculated scattered intensity of the system, taking into the pre-factors of contrast and volume fraction, and an $\mathrm{R}_{\text {sil }}$ of $80 \AA$. The Guinier region is perfectly reproduced by the model. However, in the intermediate regime, if the locations of the extinctions occur at the expected $\mathrm{q}_{1}$ and $\mathrm{q}_{2}$ values, they are much marked in the calculation. This comes from two effects: $(i)$ a possible polydispersity of the distribution of radii of nanoparticles and (ii) the convolution by the experimental resolution, which is always important for the neutrons. The final modeling of the experimental intensity (continuous line in Fig. 7a) corresponds to the form factor of spheres with a lognormal distribution with $\mathrm{R}_{0}=75 \AA$ and $\sigma=0.17$ that gives a mean radius $R_{\text {mean }}$ of $78.5 \AA$, very close to the initial estimate from $q_{1}$ and $I(q \rightarrow 0)$.

Figure $7 \mathrm{~b}$ shows the Guinier plot of the scattered intensity. The Guinier behavior is well recovered and enables to extract a gyration radius $\mathrm{R}_{g_{-} \text {sil }}$ of $72 \AA$, the validity domain $\left(q R_{g}<1\right.$, ) being thus respected down to $0.014 \AA^{-1}$. $R_{g_{-} \text {sil }}$ is lower than $R_{\text {sil }}$ as expected. The calculation of $R_{\mathrm{g}_{\text {_sil }} \text { taking }}$ into account the polydispersity with the values of the model gives $71 \AA$, in perfect accordance with the experimental data.

Finally, the Porod representation enables to obtain an estimate the surface volume $\mathrm{S} / \mathrm{V}$ from $\mathrm{I}(\mathrm{q} \rightarrow \infty) \mathrm{q}^{4} \sim 510^{26} \mathrm{~cm}^{-5} . \mathrm{S} / \mathrm{V} \sim 510^{26} /\left(2 \pi^{*}\left(3.4110^{10}--0.5610^{10}\right)^{2}\right)=510^{4} \mathrm{~cm}^{-1}$ and then $\mathrm{S}_{\text {sphere }} / \mathrm{V}_{\text {sphere }}=\mathrm{S} / \mathrm{V}^{*} \Phi_{\text {sil }}\left(1-\Phi_{\text {sil }}\right) \sim 4.2510^{6} \mathrm{~cm}^{-1}$ (or $0.042 \AA^{-1}$ ). This gives a Porod radius $\mathrm{R}_{\mathrm{P}}$ of $70 \AA$, of the order of the radii derived from others methods. 


\subsection{What can be extracted from the analysis of the structure factor}

We will focus on this part on the structure factor that probes the correlations between objects and we consider the case of a binary system of $n$ rigid centro-symmetrical objects in a continuous media, as in part 2.4.2. As mentioned in part 2.1, it will enable to measure the correlation pair function of centers of mass of objects $g_{o b j}(r)$ (that will be simplified hereafter to $g(r)$ ) in order to get a full description of the structures. Such structure is obviously set by the interactions (attractive, repulsive, long-ranged, short-ranged, etc. and thus to the potential pair interaction between objects $V(r)$ that represents the effective overall averaged interaction between objects. Obviously a system for which repulsions dominate will have a homogeneous structure and conversely an attractive system a heterogeneous structure. For very attractive systems, the objects will stick themselves to form aggregates. Although the aggregates results from interactions objects, their structure factor resembles more to a form factor than to what is classically depicted as a structure factor. The aggregates case will thus be discussed in a specific paragraph.

As a preliminary remark, please note that $\mathbf{S}(\mathbf{q} \rightarrow \infty)=\mathbf{1}$. Indeed, in the double sum of formula (27') that describes the structure factor, $e^{-i \vec{q} \cdot \overrightarrow{\alpha \beta}}=1$ when $\alpha=\beta$ and all other terms vanish when $\mathrm{q} \rightarrow \infty$. In practice, such a limit is obtained when $q$ is much larger than the inverse of the typical size of the object.

Equation (27') may also be rewritten explicitly as a function of $g(r)(19)$ :

$$
S(q)=1+\rho_{v} \int_{\text {volumeV }} g(r) e^{i \vec{q} \vec{r}} d \vec{r}=1+\rho v \int_{0}^{\infty}(g(r)-1) \frac{\sin q r}{q r} 4 \pi r^{2} d r
$$

where $\rho_{V}$ is the number density of objects $(\mathrm{n} / \mathrm{V})$. The density at a distance $r$ from the mass center of the object is thus $\rho g(r)$.

$S(q)$ and $g(r)$ are related by a simple Fourier Transform FT. However in is usually not possible to extract easily $g(r)$ by an inverse Fourier transform of experimental SANS data for two reasons. First, $S(q)$ is measured on a limited range of q. Second, it is necessary to take into account the experimental resolution, which is poor in SANS (see part 3).

There are some simple experimental cases for which the structure factor is trivial: $(i)$ for systems without any interactions, $g(r)=1$ and $S(q)=1$, (ii) for very diluted systems, id est for "gas of objects" $\mathrm{S}(\mathrm{q})=1$ because $\rho \sim 0$ and (iii) in case of crystals, $g(r)$ shows a series of peaks (which are Dirac function at zero temperature) corresponding to the positions of the objects within the crystalline lattice and $\mathrm{S}(\mathrm{q})$ shows a series of Dirac function which are the Bragg peaks of diffraction. Otherwise, and this is the most interesting case, one obtains a correlation at small $r$ and disorder at large $r$.

A very important property of $\mathrm{S}(\mathrm{q})$ is its value at 0 . As explained in 2.3.2, it is proportional to the isothermal compressibility $\chi_{T}$. In SANS, one probes objects of size which are much larger than small molecules. The dispersing medium (solvent, matrix, etc.) may be considered as a continuum and the relevant parameter to describe the pressure of objects within the continuous media is the osmotic pressure $\Pi$. The isothermal osmotic compressibility is thus:

$$
\left(\frac{\partial \Pi}{k T \partial \rho}\right)_{T}=\frac{1}{S(0)}
$$

The evaluation of isothermal compressibility immediately informs on the overall sign of interactions (repulsions/attractions) and its strength.

For repulsive systems, the system is homogeneous and has a liquid-like behavior. $\mathrm{S}(\mathrm{q})$ shows thus the following features: In the low q region, $\mathrm{S}(\mathrm{q})$ has a value lower than 1 due to its weak compressibility whose value can be extrapolated from $S(q \rightarrow 0)$. The smaller it is, the more repulsive the system is. At intermediate $\mathrm{q}, \mathrm{S}(\mathrm{q})$ shows a correlation peak at $\mathrm{q}^{*}$ followed by its harmonics, associated to the mean distance between objects with $d_{\text {mean }} \sim 2 \pi / q^{*}$. The stronger the repulsions, the higher the 
intensity of the peak (at constant $\Phi$ ) since there are less fluctuations around the mean distance between objects. At large $\mathrm{q}, \mathrm{S}(\mathrm{q})$ is $\sim 1$.

As the system is homogeneous, when the volume fraction $\Phi$ is increased (respectively decreased), the correlation peak moves towards large q (respectively low q). Indeed, the total volume is $\mathrm{V}_{\text {tot }}=$ $\mathrm{nd}_{\text {mean }}^{3}$. Given that is $\Phi=\mathrm{nV}_{\text {obj }} / \mathrm{V}_{\text {tot }}$ (where $\mathrm{V}_{\text {obj }}$ is the volume of the object), it comes that $\Phi=$ $\mathrm{V}_{\text {obj }} / \mathrm{d}_{\text {mean }}^{3}$ and thus:

$$
q^{*}=\frac{2 \pi}{v_{o b j^{1 / 3}}} \Phi^{1 / 3}
$$

Measurements at different concentrations, assuming that the form factor does not change with concentration (which is valid for rigid objects), enable to check such a dilution law and therefore the 3-D homogeneous structure of the suspensions ${ }^{* *}$. Deviation to dilution law gives information on lower dimensionality of organization.

For attractive systems, the system becomes heterogeneous. In the low q region, $S(q)$ has a value larger than 1 due to its large compressibility. S(q) progressively increases when going towards low q. The more heterogeneous the system, the larger the increase since such there are more density fluctuations within the system. In given cases, it is possible to extract a correlation size from the study of this low q-region (see next part 2.6.4). For weakly attractive systems, there is usually no correlation peak in the scattering spectra. However, for more strongly attractive systems, there is generally a correlation peak at a $\mathrm{q}^{*}$ corresponding to the most probable distance $d_{m p}$ between objects that is the contact between two objects. For spheres of radius $\mathrm{R}_{0}$, this gives $d_{m p}=2 \mathrm{R}_{0} \sim 2 \pi / \mathrm{q}^{*}$. Please keep in mind that $\mathrm{S}(\mathrm{q})$ gives a static picture averaged over all the structures adopted by the system during the measurement. The contact between two objects is not necessarily permanent. At large q, S(q) is here again $\sim 1$ by principle.

Figure 8, taken from (20), illustrates the evolution of the structure factor when tuning the interactions by adding salt in a system of spherical magnetic nanoparticles of $\gamma-\mathrm{Fe}_{2} \mathrm{O}_{3}$ that bear negative charge at their surface dispersed in an aqueous solvent. Briefly, there are Van der Waals and magnetic dipolar attractive interactions in the systems between nanoparticles which are counterbalanced by electrostatic repulsions that ensure the colloidal stability. Such repulsions may be partially screened by the addition of salt, which makes the system pass from purely repulsive when potential pair is completely dominated by electrostatics (for an ionic strength of $0.0025 \mathrm{M}$ ) to partially attractive when attractions are of the same order of magnitude (at $0.1 \mathrm{M}$ ) up to clearly attractive (at $0.18 \mathrm{M}$ ). Further addition of salt leads to precipitation. Figure 8a shows the $\mathrm{S}(\mathrm{q})$ for the different ionic strength and Fig. 8c some sketches of the system in direct space for the corresponding salinities, while Fig. 8b shows the evolution of S(q) upon an increase of $\Phi$ at constant ionic strength when the system is dominated by repulsions and therefore repulsive.

\subsubsection{Weakly interacting system: Virial expansion and second Virial coefficient system}

A very convenient way to determine the sign of potential pair and possibly an overall estimation of its intensity in diluted system is to measure the second Virial coefficient $A_{2}$ from $S(q)$. This is based on the

\footnotetext{
** Besides the canonical case of rigid centro-symmetrical presented here, it is very common to study $\mathrm{q}^{*}$ as function of concentration $c$ in order to check the homogeneity of a given system, or its regime of dilution in a polymeric system. For instance, the correlation peak observed in solutions of polyelectrolyte ( $a k a$ the "polyelectrolyte peak") varies like $c_{P E L}^{1 / 3}$ in dilute regime and $c_{\mathrm{PEL}}^{1 / 2}$ in semi-dilute regime, the threshold concentration being set at the overlapping concentration $c^{*}$ (see for instance the very nice course from Combet et al. in (48)).
} 


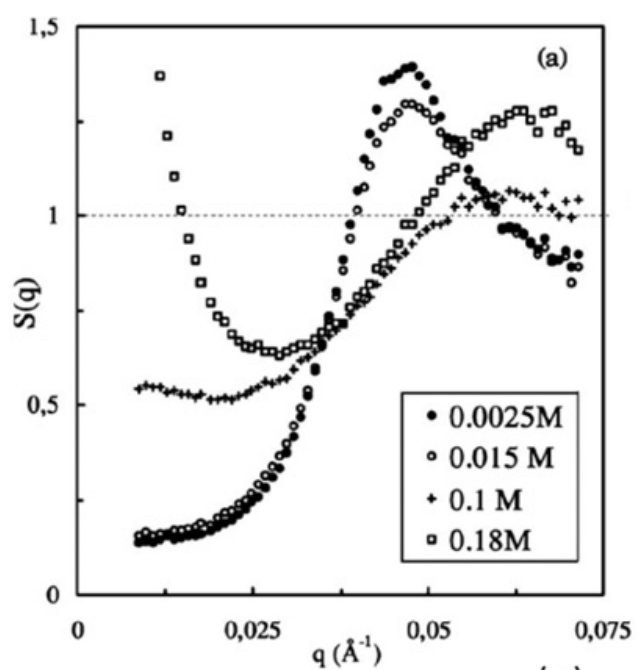

(a)

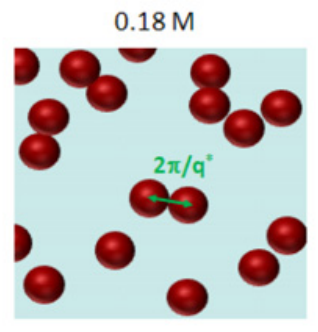

$0.1 \mathrm{M}$
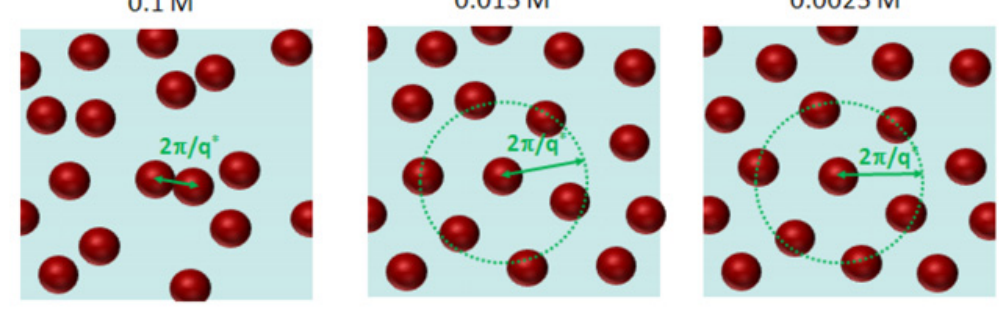

(c)

Figure 8. Structure factor for an aqueous suspension of spherical magnetic nanoparticles of $\gamma$-Fe ${ }_{2} \mathrm{O}_{3}$ (of radius around $\sim 3.5 \mathrm{~nm}$ ) for various physicochemical conditions, from reference (20). (a) $\mathrm{S}(\mathrm{q})$ as function of ionic strength at $\Phi=0.07$. (b) $\mathrm{S}(\mathrm{q})$ as function of $\Phi$ at $\mathrm{I}=0.025 \mathrm{M}$. The inset demonstrates that $\Phi=\mathrm{V}_{0 \mathrm{bj}} / \mathrm{d}_{\text {mean }}^{3}$ (see Eq. (46)). (c) Schematic pictures of the system in direct space for the different ionic strength.

description of the equation of state of the system by a Virial expansion:

$$
\Pi=\rho_{\nu} k T+\rho_{\nu}^{2} A_{2} k T+\rho_{\nu}^{3} A_{3} k T+\rho_{\nu}{ }^{4} A_{4} k T+\cdots
$$

where the second Virial coefficient $\mathrm{A}_{2}$ stands for two-bodies correlations between two objects, the third coefficient $\mathrm{A}_{3}$ for three-bodies correlations, the fourth between four-bodies $\mathrm{A}_{4}$, etc.

For a system without interactions (perfect gas), one recovers the Van't Hoff law $(\Pi=\rho v k T)$ and for diluted systems, the Virial expansion is limited to second coefficient as three-bodies correlations or higher orders are negligible $\left(\Pi=\rho_{\nu} k T+\rho_{\nu}^{2} A_{2} k T+0\left(\rho^{3}\right)\right.$. It comes that:

$$
\left(\frac{\partial \Pi}{k T \partial \rho_{\nu}}\right)_{T}=\frac{1}{S(0)} \approx 1+2 \rho_{\nu} A_{2}+0\left(\rho_{\nu}^{2}\right)
$$

Since the solution is diluted $\left(\rho_{V} \mathrm{~A}_{2}<<1\right)$, this also gives:

$$
S(0) \approx \frac{1}{1+2 \rho A_{2}} \approx 1-2 \rho_{\nu} A_{2}
$$

Some authors choose to plot either $1 / \mathrm{S}(0)$ or $\mathrm{S}(0)$. Take care on the sign! 


\section{JDN 21}

At infinite dilution, for systems interacting through isotropic interactions, $g(r)_{\rho \rightarrow 0}$ tends towards $e^{-\frac{V(r)}{k T}}$. Thus, Eq. (44) at $\mathrm{q}=0$ for very diluted systems becomes (19):

$$
S(0) \approx 1+\rho_{\nu} \int_{0}^{\infty}\left(e^{-\frac{V(r)}{k T}}-1\right) 4 \pi r^{2} d r+\cdots
$$

It comes that the second Virial coefficient is linked to the pair potential (19) by:

$$
A_{2}=\frac{1}{2} \int_{0}^{\infty}\left(e^{-\frac{V(r)}{k T}}\right) 4 \pi r^{2} d r
$$

Thus, if repulsions are dominant $(V(r)>0), A_{2}$ has a positive value. Conversely, if attractions are dominant, $A_{2}$ is negative.

In order to extract the second Virial coefficient, it is necessary to make several measurements in the low q region at different concentrations c (or densities) to obtain $S(0)$ for every concentration in order to plot $\mathrm{S}(0)$ versus $\mathrm{c}$. In this low-q region, the form factor $\mathrm{P}(\mathrm{q})$ is generally in the Guinier regime. The proper way to evaluate $\mathrm{A}_{2}$, as well as $\mathrm{R}_{g}$ (that may vary one concentration to another) is to make a Zimm Plot where all measurements are plotted like $c /(\mathrm{I}(q, c))$ versus $q^{2}$ to obtain $R_{g}$ and $\mathrm{S}(0)$ for every concentration, given that $P_{\text {Guinier }}(q) \approx 1-\frac{q^{2} R_{g}^{2}}{3} \approx \frac{1}{1+\frac{q^{2} R_{g}^{2}}{3}}$. Such a plot is for instance presented in chapter 22 of (5).

\subsubsection{Simulations}

There is nowadays a strong development of simulations (Monte-Carlo, molecular dynamics,) which is very convenient as it gives by principle the exact link between $g(r)$ and $V(r)$. The main limit is set by the size of the simulation box L which is necessarily limited and does not allow to probe large $\mathrm{r}$, and therefore low q's. It is also not very well adapted to the standard treatment of many experimental spectra as every simulation is time consuming by itself. For further information on simulations and neutrons you may read the following special issue from the SFN collection (21).

\subsubsection{Integral equations}

A proper way to make the link between $g(r)$ (or $\mathrm{S}(\mathrm{q})$ ) and $V(r)$ is to use the integral equations. This is a bit complex and out of scope of this course. We will thus only recall shortly its principle. An interested reader may find some very good introduction to it in the course of Luc Belloni (19) that is especially well written.

The basis of this method is based on the Orstein-Zernike equation that states that $g(r)$, or more precisely to the total correlation function $h(r)$ (defined by $g(r)-1$ ), is decomposed in a direct part, the direct correlation function $c(r)$, and an indirect part which is the convolution between these two functions and stands for the correlations occurring respectively via the $3^{\text {rd }}$ object, the $4^{\text {th }}$ object, ... or the $n^{\text {th }} \ldots$ object):

$$
h(r)=c(r)+\rho v \int h\left(r^{\prime}\right) c\left(\left|\vec{r}-\vec{r}^{\prime}\right|\right) d \overrightarrow{r^{\prime}}
$$

The direct correlation function $c(r)$ behaves like $e^{-\frac{V(r)}{k T}}-1$ at infinite dilution and like $-\frac{V(r)}{k T}$ at very long range (when interactions become very weak).

In Fourier space, the convolution product becomes a simple product and therefore:

$$
S(q)=1+\rho H(q)=1 /\left(1-\rho_{\nu} C(q)\right)
$$




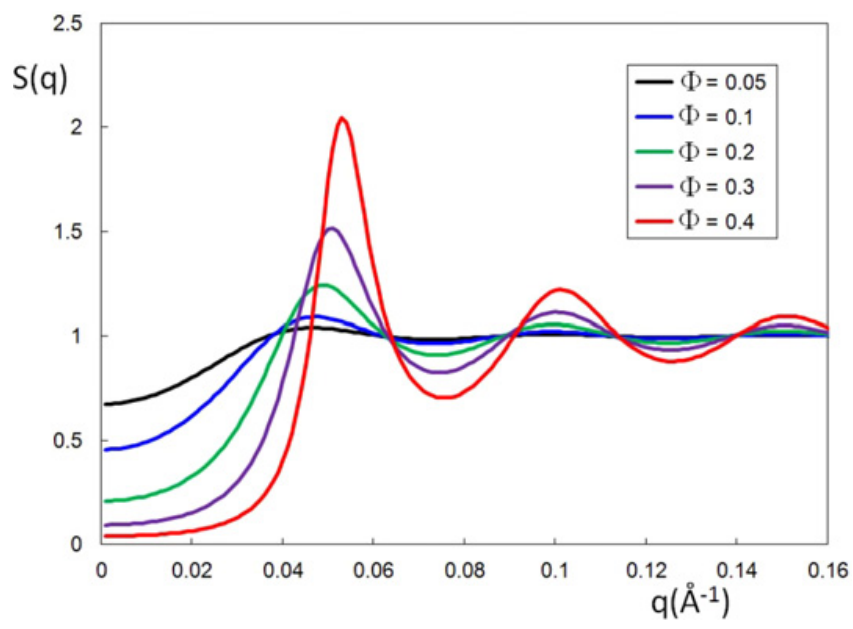

Figure 9. Calculated $\mathrm{S}(\mathrm{q})$ for a system of monodisperse hard spheres of diameter $\mathrm{d}_{0}=125 \AA$ at various $\Phi$ in the Percus-Yevick approximation.

In order to solve the problem, a second equation is necessary since two functions are unknown. Such a second relation is the closure relation, or integral equation:

$$
g(r)=\exp \left[-\frac{V(r)}{k T}+h(r)-c(r)+b(r)\right]
$$

In previous equation, the bridge function $b(r)$ is unknown. The aim of statistical physics of liquids is then to get a good solution for such a bridge function. Some approximations exist, that define different integral equations. The most famous ones are the HNC equation (Hypernetted chain), for which $b(r)$ has been suppressed $\left(g(r)=\exp \left[-\frac{V(r)}{k T}+h(r)-c(r)\right]\right)$, which is well suited for electrostatic systems; the equation PY (Percus-Yevick), for which the term $h(r)-c(r)$ has been linearized with respect to the HNC one $\left(g(r)=\exp \left[-\frac{V(r)}{k T}\right](1+h(r)-c(r))\right)$, and that described well hard-spheres systems; and finally the MSA (Mean Spherical Approximation), for which the whole exponential term of HNC has been linearized $\left(c(r)=-\frac{V(r)}{k T}\right)$. All these successive simplifications require to use such integral equations only for adequate pair potentials $V(r)$, otherwise it leads to large errors. The equations PY and MSA are often used as they have analytical forms. PY is often used to treat hard spheres potentials (or sticky potentials with short attractions range) and MSA for electrostatic systems following (22) since it enables to account for the Yukawa potential that describes the electrostatic part of the famous DLVO potential that is generally used to describe the behavior of charged colloids (the short-range Van der Waals part of the potential of the DLVO being neglected). The proper use and limits of these equations are very well discussed in (19), as well as further discussion on charged systems. The analytical forms of S(q) calculated by PY and MSA are very lengthy and are thus not reproduced here but can be found in chapter 32 of (5). As an illustration, Fig. 9 shows the structure factor of a system of spheres interacting through hard-core interactions for various volume fractions calculated with PY.

\subsubsection{Attractive systems: Correlation length}

In heterogeneous systems (gels, interpenetrated aggregated systems, binary systems close a demixion, etc.), it is difficult to extract a typical size in the system. However, it is possible to obtain a correlation length $\xi$ in the system (for instance when the system is close to a coexistence line) assuming that $g(r)$ 
has a fast decay like $g(r) \propto \frac{e^{-r / \xi}}{r} . \mathrm{S}(\mathrm{q})$ is then a Lorentzian:

$$
S(q) \propto \frac{1}{q^{2}+\xi^{-2}}
$$

$\xi^{-2}$ can thus be extracted from the interpolation at $1 / \mathrm{S}(\mathrm{q}=0)$ in a $1 /\left(\mathrm{S}(q)\right.$ versus $q^{2}$ plot.

\subsubsection{Aggregates}

We consider here the case of aggregates and focus for sake of simplicity on those made of spherical objects of volume $\mathrm{V}_{\text {obj. }}$. The scattered intensity allows a fine characterization of their aggregation number $\mathrm{N}_{\mathrm{agg}}$ if they have a finite size, id est if their overall size is lower than $1 / \mathrm{q}_{\mathrm{min}}$, of their fractal dimension $\mathrm{D}_{\mathrm{f}}$ and on their compactness.

The aggregation number $N_{\text {agg }}$ can be obtained very simply from the measurement of the mass of aggregates (through I(q $\rightarrow 0$ ), see Eq. (30)) if the volume fraction of the objects $\Phi_{\text {obj }}$ and volume of objects $\mathrm{V}_{\text {obj }}$ are known, whatever the shape of the aggregate. Indeed, since the spheres touch themselves, Eq. (30) may be applied as the aggregate forms a single object and one gets:

$$
I(q=0)=\left(\rho_{o b j}-\rho_{\text {media }}\right)^{2} \Phi_{o b j} V_{a g g}=\left(\rho_{o b j}-\rho_{\text {media }}\right)^{2} \Phi_{o b j} N_{a g g} V_{o b j}
$$

where $V_{\text {agg }}$ is the volume of the aggregate. Experimentally, it is sometimes possible to measure both the respective scattering from isolated object and those from aggregates (for instance when aggregation is induced by a parameter such as the addition of salt, an appliance of a magnetic field, drying of solvent, etc.). In this case $N_{a g g}$ is simply obtained by the division of the two spectra for I(q $\left.\rightarrow 0\right)$ (taking obviously into account the contrast and volume fraction terms).

For very dense aggregates of finite size, the inner of the aggregate is rather homogeneous from the point of view of SLD. The scattering resembles then to the form factor of the object whose shape corresponds to the envelop of the aggregate (which is very often a sphere) in the Guinier region at low $\mathrm{q}$ and at intermediate q. At larger q, as all objects have numerous contacts with other ones in the inner of the aggregate, there is also a correlation peak corresponding to the contact between objects (at $\mathrm{q}^{*} \sim$ $2 \pi / 2 \mathrm{R}_{0}$ for spheres of radius $\mathrm{R}_{0}$ ). The intensity of this correlation peak increases with $N_{\text {agg }}$ because the mean number of contacts increases. When the shape of the scattering curve can be modeled by a form factor, it gives access to the volume of the aggregate $V_{a g g}$. The compactness of the aggregate is then directly derived via $N_{a g g} \mathrm{~V}_{o b j} / V_{a g g}$.

For open aggregates, it is not possible to model the scattering by a form factor. In the intermediate regime q, the scattered intensity shows a power law decay whose exponent gives to the fractal dimension of the aggregates if observed on a sufficiently large q-range, at least one decade to make sense. Such fractal dimension $\mathrm{D}_{\mathrm{f}}$ is linked to the process of aggregation between the objects. For spheres interacting through an isotropic potential, $\mathrm{D}_{\mathrm{f}}$ is 1.78 in case of Diffusion Limited Aggregation (DLA) and 2.1 for Reaction Limited Aggregation (RLA) (23) (24). DLA occurs when aggregation is only limited by diffusion processes and aggregation occurs at contact ("stuck when touched") while RLA occurs when objects have to overcome a barrier potential to aggregates, for instance in case of weakly charged systems. For anisotropic objects such an cylinders, $\mathrm{D}_{\mathrm{f}}$ increases with the aspect ratio of objects up to 2.3 in case of RLA for highly anisotropic rods (25). The correlation peak corresponding to the contact between objects is usually hardly visible. For aggregates of finite size, it is possible to estimate an overall size of aggregates $R_{a g g}$ from $N_{a g g}, \mathrm{D}_{\mathrm{f}}$ and $\mathrm{V}_{o b j}$. Indeed, from the definition of a mass fractal (see Eq. (37)), it comes that $R_{a g g}=N_{a g g}{ }^{1 / D_{\mathrm{f}}} R_{o b j}$.

The typical features of aggregates of the scattered intensity of aggregates are illustrated in Fig. 10. It shows the hierarchical structure observed in a nanocomposite system made of spherical magnetic nanoparticles of $\gamma-\mathrm{Fe}_{2} \mathrm{O}_{3}$ of around $10 \mathrm{~nm}$ in diameter dispersed in a polystyrene (PS) matrix (26). At low volume fraction $\left(\Phi_{\text {part }}=10^{-5}\right)$, dense primary aggregates composed of a few tens of nanoparticles 


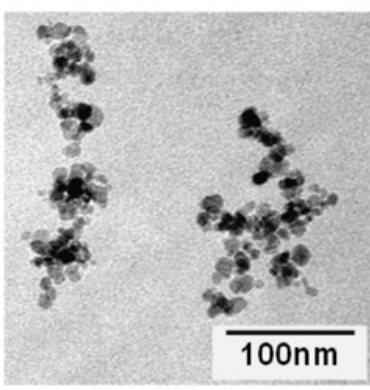

(a)

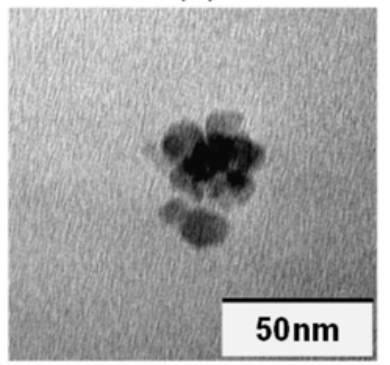

(b)

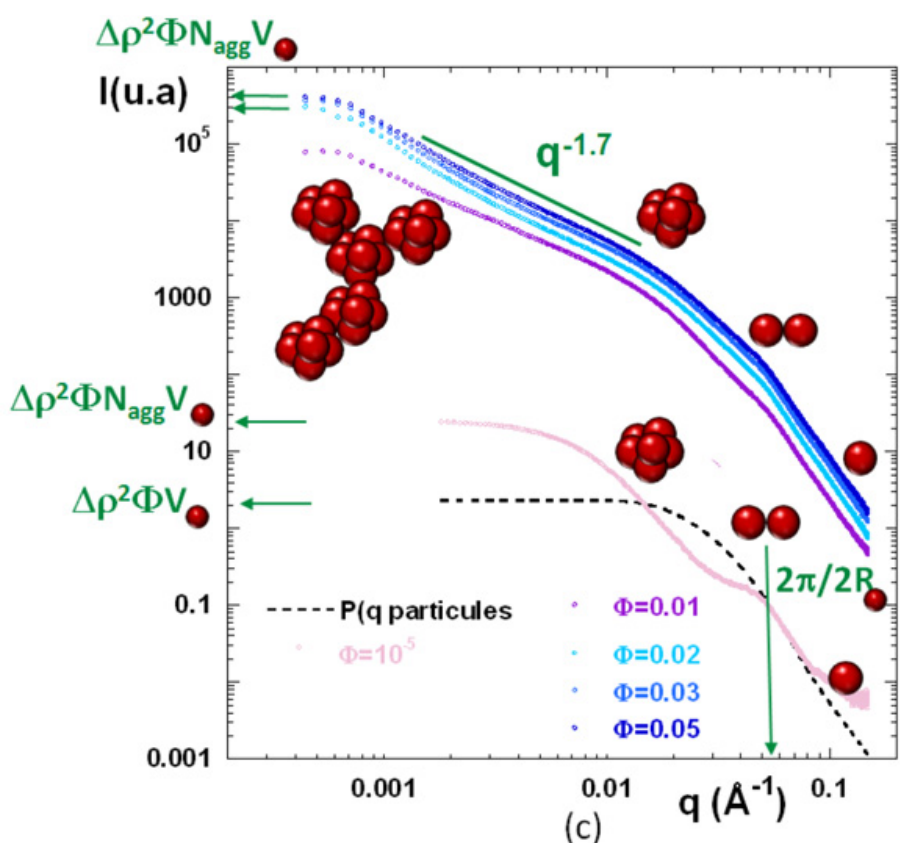

(c)

Figure 10. Structural characterization of a nanocomposite system made of spherical magnetic nanoparticles of $\gamma-\mathrm{Fe}_{2} \mathrm{O}_{3}$ of around $10 \mathrm{~nm}$ in diameter dispersed in a polystyrene (PS) matrix at different volume fractions (26). (a) TEM picture for a sample at $\left(\Phi_{\text {part }}=510^{-4}\right)$; (b) TEM picture for a sample at $\left(\Phi_{\text {part }}=10^{-5}\right)$; (c) Scattered intensity at various $\Phi_{\text {part }}$ and main features of the scattering spectra.

are formed: $\mathrm{I}(\mathrm{q})$ superimpose to the form factor of the nanoparticle at very large $\mathrm{q}$, shows a strong correlation peak at $0.06 \AA^{-1}$ originating from the contact between nanoparticles, a strong decay at intermediate $\mathrm{q}$ (between $\mathrm{q}^{-3}$ and $\mathrm{q}^{-4}$ ) showing that nanoparticles form dense 3-D aggregates and a plateau in the Guinier region. At higher volume fraction, these dense primary aggregates aggregate themselves at larger scale to form a hierarchical structure. The scattering in the large $\mathrm{q}$ region is then similar to those obtained at low volume fraction case but differ in the low q region. The former Guinier region is replaced by a $\mathrm{q}^{-1.7}$ scattering decay, reminiscent of the RLA process, showing that primary aggregates have aggregated themselves. Finally, at much smaller q, a Guinier plateau is reached, enabling to obtain the overall aggregation number of these "open aggregates of dense aggregates". Such structures are perfectly recovered by TEM pictures of the system in real system. Please remember that these TEM picture cannot provide any quantitative characterization of the system.

\subsection{Contrast variation}

We will give a brief overview here on the possibilities offered by neutrons to finely tune the scattering signal by playing on the value of the SLD $\rho_{i}$ of the elementary scatterers in the system to either enhance or extinct some parts of the signal to perform refined studies, these tricks being known as contrast variation methods. This is based on the fact that the coherent scattering length varies from one isotope to another for neutrons, that atoms which are located in adjacent places in the periodic table may have very different coherent scattering length and, above all, that the relevant parameter for the contrast term in SANS is the SLD and not the coherent scattering length. The SLD of an elementary scatter can thus be tuned either by partial or total specific substitution. The most famous (and used!) case is the one of hydrogen. ${ }^{1} \mathrm{H}$ and deuterium ${ }^{2} \mathrm{H}$ that have very different values (see Table 2 that presents the $\mathrm{b}_{i}$ of 
Table 2. Coherent scattering length of most common atoms found in organic compounds and scattering length density of some representative molecules. PS refers to PolyStyrene.

\begin{tabular}{|l|c|c|c|c|}
\hline \multicolumn{5}{|c|}{ Coherent scattering length $\mathrm{b}\left(10^{-12} \mathrm{~cm}\right)$} \\
\hline${ }^{1} \mathrm{H}$ & $\mathrm{D}\left({ }^{2} \mathrm{H}\right)$ & $\mathrm{C}$ & $\mathrm{O}$ & $\mathrm{N}$ \\
\hline-0.374 & 0.667 & 0.665 & 0.580 & 0.936 \\
\hline \multicolumn{5}{|c|}{ Scattering length density $\left(10^{10} \mathrm{~cm}^{-2}\right)$} \\
\hline $\mathrm{H}_{2} \mathrm{O}$ & $\mathrm{D}_{2} \mathrm{O}$ & $\begin{array}{c}\text { Mixture of } \mathrm{H}_{2} \mathrm{O} \\
\text { and } \mathrm{D}_{2} \mathrm{O} \text { with a given } \Phi_{\mathrm{H} 2 \mathrm{O}}\end{array}$ & $\mathrm{PS}_{\mathrm{H}}$ & $\mathrm{PS}_{\mathrm{D}}$ \\
\hline-0.56 & 6.38 & $-0.56 \Phi_{\mathrm{H} 2 \mathrm{O}}+6.38\left(1-\Phi_{\mathrm{H} 2 \mathrm{O}}\right)$ & 1.41 & 6.5 \\
\hline
\end{tabular}

the most common elements of organic compounds and the SLD of some representative molecules). Although it is in practice done most of the times on hydrogen, it is not limited to such an atom. For instance, $\mathrm{b}\left({ }^{58} \mathrm{Ni}\right)$ is $1.410^{-12} \mathrm{~cm}$ (one of the highest of the periodic table) and $\mathrm{b}\left({ }^{62} \mathrm{Ni}\right)$ is -0.87 $10^{-12} \mathrm{~cm}$. Also, a solvent made of the mixture of hydrogenated and deuterated molecules of the same chemical species behaves as a single solvent from point of view of physical properties, the SLD of the mixture being the average of the scattering length density of the objects weighted by their respective volume fraction (see the example for mixture of light and heavy water in Table 2).

The contrast variation methods may be used with three different strategies that will be depicted hereafter: $(i)$ creating some contrast to probe some molecules within an homogenous phase such as a polymer melt; (ii) match selectively the scattering of a given component in a ternary system by adjusting its SLD to the one of the second one to probe only the scattering of the third; (iii) make mixtures of hydrogenated and deuterated objects to separate the form factor and the structure factor within the scattering intensity.

\subsubsection{Selective deuteration to create some contrast within an homogenous phase}

In the previous parts, we have implicitly considered that there was an existing contrast between the objects to be characterized and the continuous phase. In practice, this is not the case in two situations: (i) when one wants to probe an object within a continuous phase made of the same objects or (ii) when the SLD of the continuous phase is exactly (or almost...) similar to the SLD of the object. The first case occurs typically for the characterization of a polymeric chain within its own polymeric melt and the latter when characterizing an organic compound within a solvent that has a very close chemical composition (for instance when probing polystyrene PS within toluene given that the chemical structure of a repetition unit of PS is exactly similar to the one of a toluene molecule). The system is thus homogeneous from a SLD point of view. In order to create a contrast within the system, the SLD of one of the two components has to be changed by isotopic substitution, generally the deuteriation of hydrogen atom (see Fig. 11). This is very easy to achieve experimentally when one component is a solvent, otherwise deuteration of a given molecule has to be done specifically (see part 3.4).

In both cases it is assumed that deuteration does not change the physical properties of the system. This is generally true, but such properties can be changed, in particular if they are linked to hydrogen bonding. For instance, the properties of $\mathrm{D}_{2} \mathrm{O}$ are slightly changed with respect to $\mathrm{H}_{2} \mathrm{O}$ (melting point is at $4{ }^{\circ} \mathrm{C}$, maximum of density at $11^{\circ} \mathrm{C}$, pD is equal to $\left.\mathrm{pH}+0.4 \ldots\right)$. Also, the Flory-Huggins interaction parameter $\chi$ of a hydrogenated monomer with is deuterated counterpart is not necessary null $\left(\chi=1.710^{-4}\right.$ for the couple $\left.\mathrm{PS}_{\mathrm{H}} / \mathrm{PS}_{\mathrm{D}}\right)(27)$. Such modifications have to be taken into account for data interpretation.

Form factor within the polymer melt

In the case of the introduction of deuterated object within similar hydrogenated objects, only the form factor is measured, whatever the content in deuterated objects. Let us write $x_{D}$ the volume fraction of deuterated objects. Since both deuterated and hydrogenated elementary scatterers have the same 

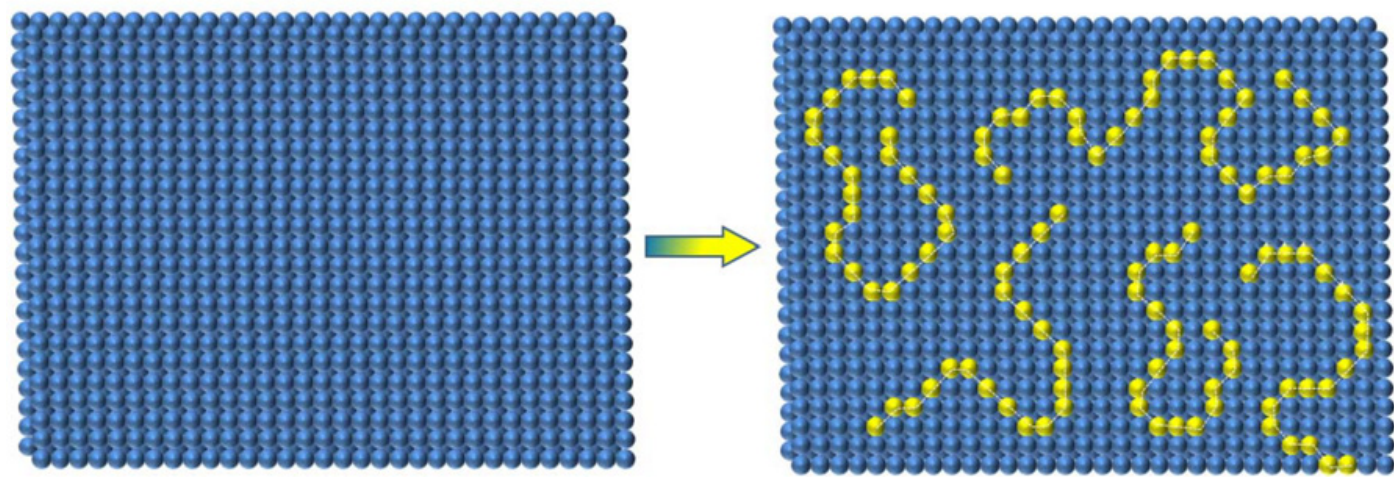

Figure 11. Creating some contrast within a polymer blend by isotopic substitution to enable a SANS characterization. Left panel: all repetition units are hydrogenated, it is not possible to distinguish is a repetition unit belong to a chain or another, the blend is an homogeneous media; Right panel, the deuteration of some chains reveal them and allows their characterization.

volume, the general Eq. (18) can be written either from the point of view of deuterated or from one of hydrogenated objects which have the same form factor $\mathrm{P}(\mathrm{q})$ and structure factor $\mathrm{Q}(\mathrm{q})$ :

$$
\begin{gathered}
I(q)=\left(\rho_{D}-\rho_{H}\right)^{2} v_{o b j}{ }^{2} S_{D D}(q)=\left(\rho_{D}-\rho_{H}\right)^{2} v_{o b j}{ }^{2}\left[x_{D} n N^{2} P(q)+x_{D}{ }^{2} n^{2} N^{2} Q(q)\right] \\
I(q)=\left(\rho_{D}-\rho_{H}\right)^{2} v_{o b j}{ }^{2} S_{H H}(q)=\left(\rho_{D}-\rho_{H}\right)^{2} v_{o b j}\left[\left(1-x_{D}\right) n N^{2} P(q)+\left(1-x_{D}\right)^{2} n^{2} N^{2} Q(q)\right]
\end{gathered}
$$

It comes that: $x_{D} P(q)+x_{D}^{2} n Q(q)=\left(1-x_{D}\right) P(q)+\left(1-x_{D}\right)^{2} n Q(q)$ and thus that:

$$
P(q)=-n Q(q)
$$

The intensity finally writes:

$$
I(q)=\left(\rho_{D}-\rho_{H}\right)^{2} v_{o b j}{ }^{2} x_{D}\left(1-x_{D}\right) n N^{2} P(q)
$$

Experimentally, the maximum of intensity is obtained for $x_{D}=0.5$ ("theorem 50/50") (28).

\subsubsection{Contrast variation in ternary system}

It is very often very interesting to study a system made of three distinct kinds of elementary scatterers. This situation appears when one aims at characterizing a mixture of two species in a continuous phase, or at characterizing a complex object made of two distinct parts with different SLD in a continuous phase, as for example a colloidal suspension made of inorganic nanoparticles grafted by polymeric chains for which is it interesting to probe separately the inorganic core and the polymeric corona (29) $(30)^{\dagger \dagger}$. In such ternary system, the general equation of scattering (18) writes:

$$
I(q)=k_{2}^{2} S_{22}(q)+k_{2} k_{3} k_{23}(q)+k_{3}^{2} S_{33}(q)
$$

with $k_{\alpha}=b_{\alpha}-b_{1} \frac{v_{\alpha}}{v_{1}}$.

\footnotetext{
$t^{\dagger}$ The contrast variation technique is especially suited for the study of suspensions of hairy spherical nanoparticles because the structure factor is similar in both contrasts. In the "core" contrast, the form factor of the object is generally trivial to model as it is the one of a smooth sphere, the experiment enables thus to extract the structure factor to determine the overall organization of the hairy objects. On the reverse, the measurement in the "shell" contrast enables the fine characterization of the polymeric shell as it allows a very accurate determination of the form factor once the scattered intensity has been divided by the structure factor.
} 


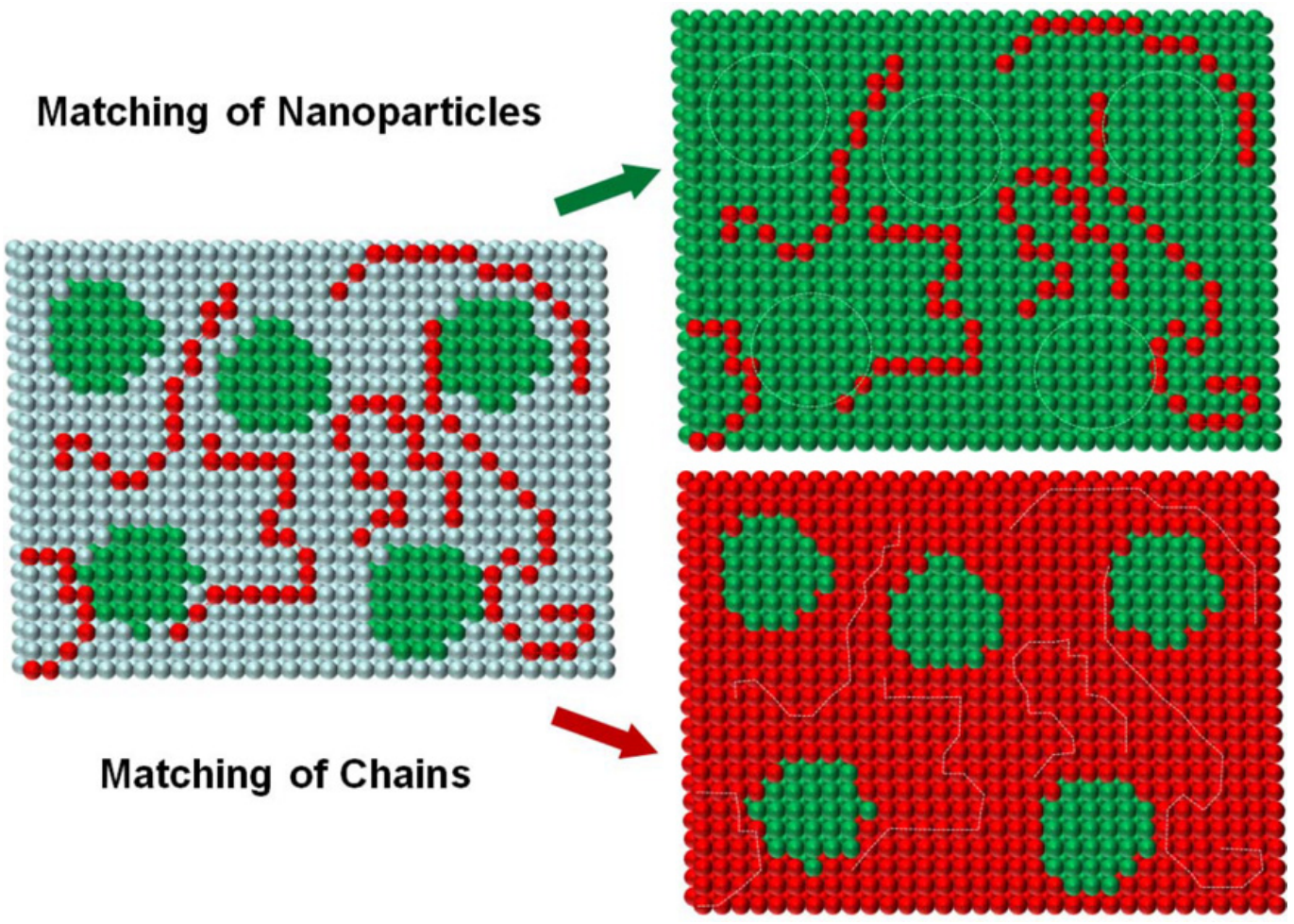

Figure 12. Principle of contrast matching in a ternary system made of a mixture of nanoparticles and polymeric chains in a solvent (left panel). When the SLD of the solvent is adjusted to the one of the nanoparticles, only the chains are visible (top right panel). Similarly, when the SLD of the solvent is adjusted to the one of the chains, only the nanoparticles are visible (bottom right panel).

The interpretation of the scattering spectra is almost impossible as it is the sum of three unknown partial structure factors. However, if the SLD of the continuous phase $\rho_{1}\left(b_{1} / v_{1}\right)$ can be continuously tuned and set to a value for which $k_{2}=b_{2}-b_{1} \frac{v_{2}}{v_{1}}=0$, then Eq. (60) reduces to $I(q)=k_{3}{ }^{2} S_{33}(q)$. Similarly, for $k_{3}=b_{2}-b_{1} \frac{v_{2}}{v_{1}}=0, I(q)=k_{2}^{2} S_{22}(q)$. These specific values of $\rho_{1}$ are the contrast matching conditions. It comes then possible to analyze the SANS data following parts 2.5 and 2.6, for which we considered the case of the a two-components system since the ternary system becomes a binary system from the SLD point of view (see illustration for a mixture of nanoparticles and chains in a solvent in Fig. 12 or for an emulsion in Fig. 13). Moreover, once $S_{22}(\mathrm{q})$ and $S_{33}(\mathrm{q})$ are determined at the contrast matching conditions, the partial structure $S_{23}(\mathrm{q})$ depicting correlations between the two species can be obtained by a measurement at a third contrast conditions for which $k_{2} \neq 0$ and $k_{3} \neq 0$.

Such elegant experiments require that the SLD of the continuous phase $\rho_{1}$ can be continuously tuned on a very large range. Fortunately, when this continuous phase is either water or a common organic solvent, its SLD varies on a range that covers almost all existing materials when passing from fully hydrogenated to fully deuterated solvent molecules owing to the strong difference of coherent scattering length between ${ }^{1} \mathrm{H}$ and ${ }^{2} \mathrm{H}$. Please note that it is possible to set $\rho_{1}$ to 0 for water (in a $8 \% \mathrm{D}_{2} \mathrm{O} / 92 \% \mathrm{H}_{2} \mathrm{O}$ mixture), thus contrast variation can be used in complex systems containing air such as foams.

In order to illustrate the principle of contrast variation, we show here an example extracted from reference (31) that aims at characterizing gels made of aqueous mixtures of lysozyme, a small positively charged globular protein, whose scattering can be matched in a mixture $98 \% \mathrm{D}_{2} \mathrm{O} / 2 \% \mathrm{H}_{2} \mathrm{O}$ mixture, and 


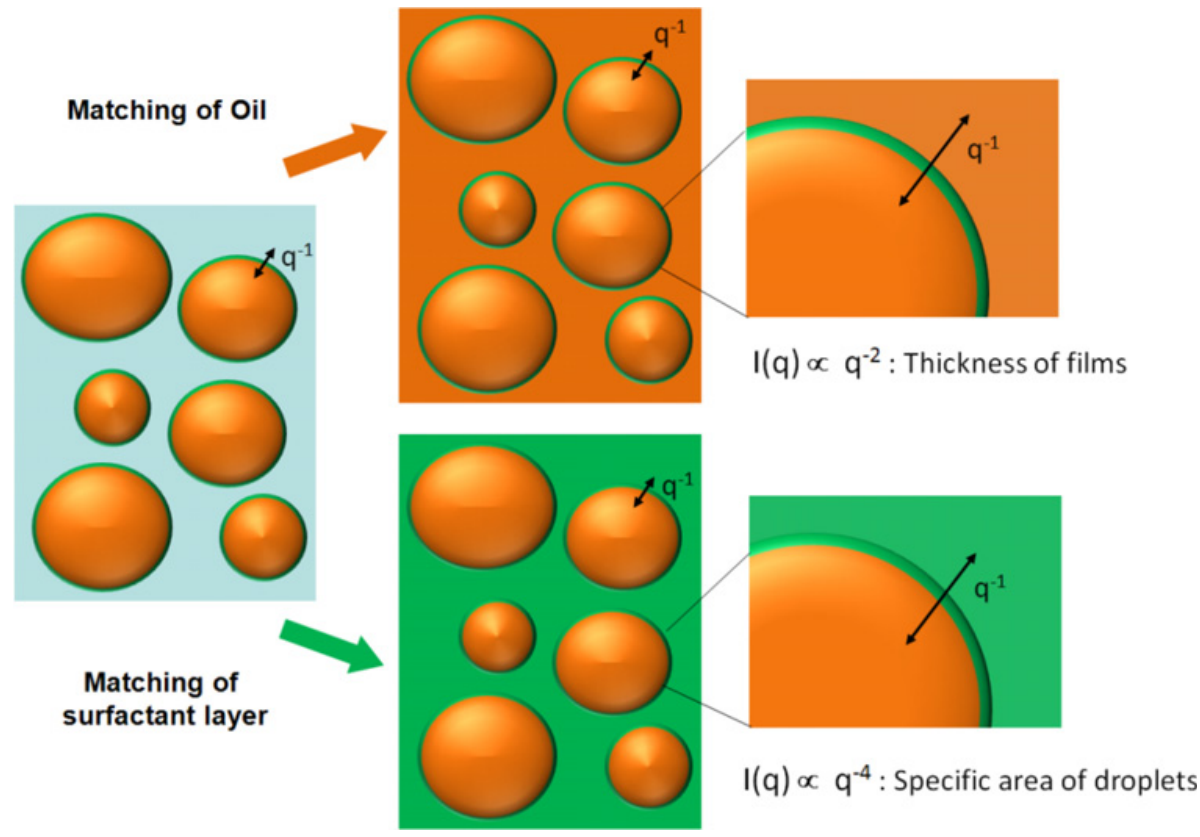

Figure 13. Principle of contrast matching in an emulsion. The radius of a droplet being much larger than the scale probed by neutrons, only the local surface of such droplet is probed. When the SLD of the solvent is adjusted to the one of oil, only the surfactant layers is probed that resembles locally to a plan and it thickness is measured. When the SLD of the solvent is adjusted to the one of surfactant, the droplet appear smooth to neutrons and specific area of the droplet is measured according to Porod law.

PolyStyrene Sulfonate (PSS), a negatively charged polyelectrolyte, whose scattering can be matched $43 \% \mathrm{D}_{2} \mathrm{O} / 57 \% \mathrm{H}_{2} \mathrm{O}$. Both components can thus be easily matched, as in Fig. 12. Figure 13 shows the scattered intensity of the system in both contrast conditions, either when PSS is matched (Fig. 14a), or when lysozyme is matched (Fig. 14b), where they are compared with the scattering spectra of individual systems (in black) at the same concentrations within the same $\mathrm{D}_{2} \mathrm{O} / \mathrm{H}_{2} \mathrm{O}$ mixtures. It immediately appears that both species are organized in a very different way within the mixture.

Briefly, the main features of scattering spectra enable to picture the refined structure of the gel. The scattering of the pure solution of PSS chains decays like $\mathrm{q}^{-1}$ at large $\mathrm{q}$, as observed for a factor of a rigid rod, because of repulsions between adjacent charges along the backbone rigidify the chain. At small q, the scattering decays strongly when going towards low q, showing that the osmotic compressibility is very weak due to strong electrostatic repulsions between chains. Such strong repulsions organize the chains in an homogeneous transient network and it appears at intermediate $\mathrm{q}$ a strong correlation peak whose $\mathrm{q}^{*}$ position is linked to the mesh size of the network $\xi\left(\mathrm{q}^{*}=2 \pi / \xi\right)$. In presence of proteins, the main features of the scattering are recovered, showing that the overall organization of the PSS chains is not altered by protein, except that $\mathrm{q}^{*}$ has been strongly shifted towards low q. Such large increase of the mesh size suggests a strong shrinking of the chains at local scale owing to mass conservation, and thus that chains are partially rolled around the proteins. The scattering of the pure solution of proteins resembles to the form factor of spherical objects with a Guinier plateau at low $\mathrm{q}$ and a $\mathrm{q}^{-4}$ scattering decay at large q due to the globular shape the proteins. There is no structure factor, the system behaving thus like at a perfect gas. In presence of PSS chains, the scattering of the proteins superimpose with such form factor at large q, there is thus no specific organization at the local scales. At the lowest q, the scattering varies strongly with a $\mathrm{q}^{-2.5}$ decay, revealing large density fluctuations of proteins within the 


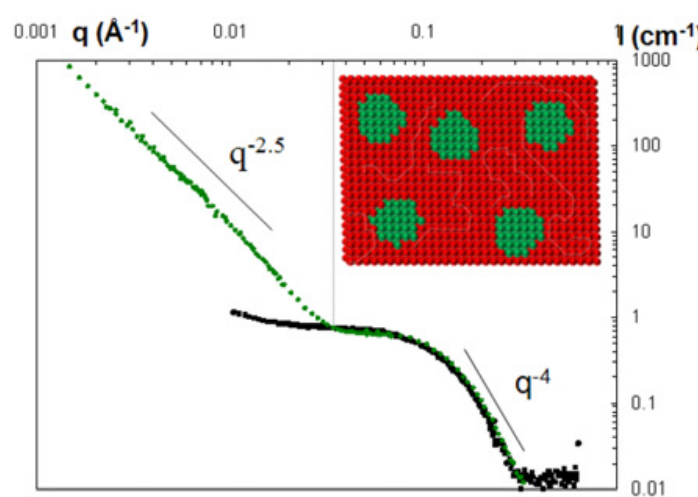

(a)

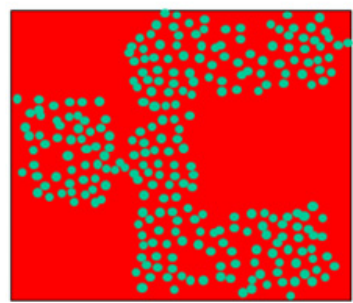

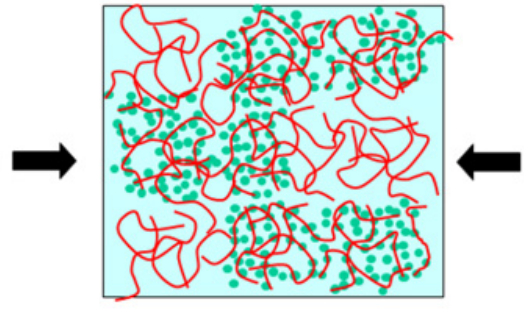

(c)

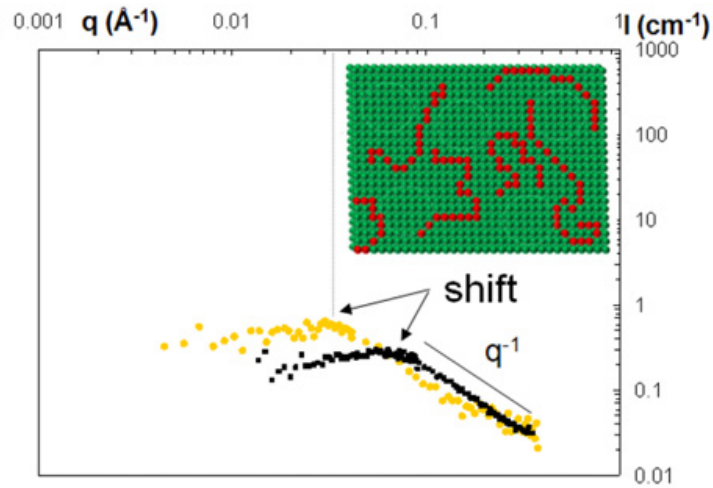

(b)

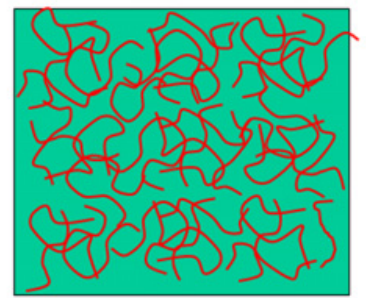

Figure 14. Illustration of contrast matching on gels made of an aqueous mixture of positively charged globular proteins (in green on the sketches) and negatively charged polyelectrolyte chains (in red in the sketches) from (31). (a) Scattering of the system when chains are matched (green curve) compared to the system of pure proteins at the same concentration in the same contrast conditions (black curve); (b) Scattering of the system when proteins are matched (yellow curve) compared to the system of pure chains at the same concentration in the same contrast conditions (black curves); (c) Schematic structure of the system combining partial structures from the two components extracted from both contrast conditions.

gel which are organized with a fractal dimension of 2.5. Remarkably, the change of scattering regimes occurs exactly at the $\mathrm{q}^{*}$ of the correlation peak in the other contrast. The heterogeneities of distributions proteins of proteins occur at larger scale than the mesh size of the network. The gel can thus be described schematically as containing two types of meshes with a very heterogeneous distribution, one filled with protein and the other not (Fig. 13c), and that the meshes contain proteins crosslink all the PSS network to form a macroscopic gel.

This example demonstrates the power of the contrast variation technique as is shows that the extinction of the scattering signal can be achieved very neatly. Indeed, on Fig. 13, the scattering of the lysozyme when PSS chains are matched is 1000 times higher than those of PSS chains when proteins are matched while the contrast terms $\left(\rho_{\text {specie }}-\rho_{\text {solvent }}\right)^{2}$ are similar in both cases by symmetry! Also it enables to demonstrate that within the mixture co-exist a very homogeneous system (PSS network chains) and a very heterogeneous system (proteins acting as cross-linkers).

More than 3 components?

The contrast matching technique is very suited for ternary systems. However it is possible to characterize a 4-components system when 2 objects have the same SLD, so they can be matched together in order to give access to the lone scattering of the third one (32). 

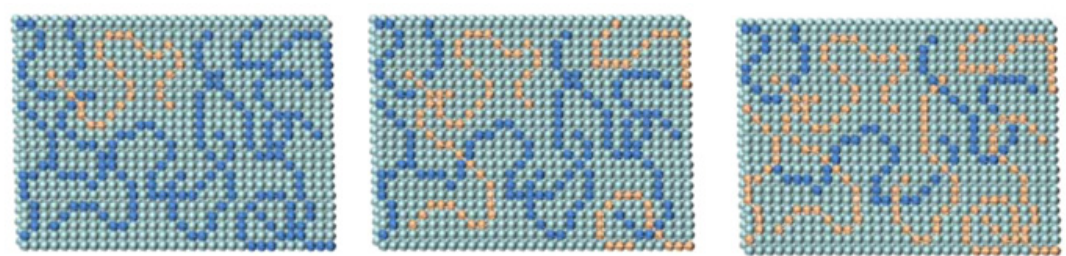

(a)
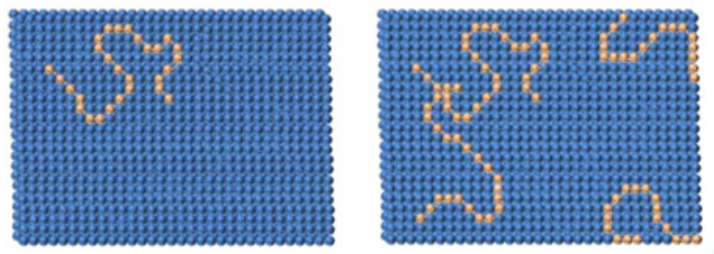
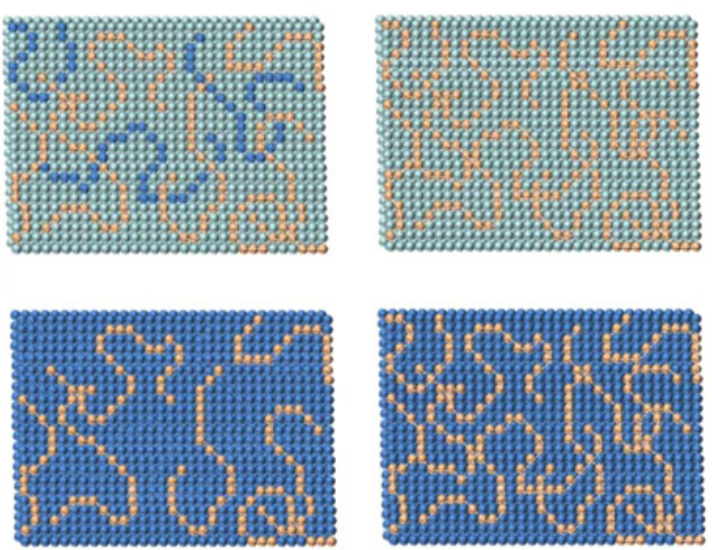

(b)

Figure 15. Principle of extrapolation to zero concentration method. Several mixtures of deuterated and hydrogenated objects are made with the same number of object but a variable content in deuterated objects. The partial structure factors are the same in all samples. (a) When the SLD $\rho_{1}$ of continuous phase is different from $\rho_{H}$ and $\rho_{D}$, all contributions are visible. (b) When $\rho_{1}=\rho_{H} ; \mathrm{S}_{D D}(\mathrm{q})$ is visible: the structure factor $\mathrm{Q}(\mathrm{q})$ progressively vanishes when the content in deuterated objects is decreased.

\subsubsection{Separating form factor and structure factor for a system objects in a continuous phase}

We showed in part 2.4.2 how the respective contributions to the scattering of the structure factor and of the form factor object can be decoupled easily for centro-symmetrical objects (see part 2.4.2) in a continuous phase. This remains in practice limited to the study of systems of nanospheres (micelles, inorganic nanospheres... ). We will show here how it is possible to decouple the structure factor and the form factor thanks to contrast variation whatever the system and without any prior assumption on the form factor and structure factor. This is especially important for the study of polymeric systems.

Two methods are possible which are both are based on the principle of making measurements on mixtures of deuterated and hydrogenated of the same objects with a variable content of deuterated objects $x_{D}$ objects and to choose craftily the SLD of the continuous phase.

Following Eq. (18), the scattered intensity of a mixture writes:

$$
I(q)=b_{H}^{2} S_{H}(q)+2 \cdot b_{H} b_{D} b_{H D}(q)+b_{D}^{2} S_{D}(q)
$$

Both form factor and structure factor may vary with $\mathrm{n}$. The partial structure factors write:

$$
\begin{aligned}
S_{D}(q) & =x_{D} n N^{2} P(q, n)+x_{D}{ }^{2} n^{2} N^{2} Q(q, n) \\
S_{H D}(q) & =x_{D}\left(1-x_{D}\right) n^{2} N^{2} Q(q, n) \\
S_{H}(q) & =\left(1-x_{D}\right) n N^{2} P(q, n)+\left(1-x_{D}\right)^{2} n^{2} N^{2} Q(q, n)
\end{aligned}
$$

In the first method, called interpolation to zero concentration (Fig. 14), the idea is to $(i)$ make several measurements on different mixtures with the same overall concentration at constant $n$ but different $x_{D}$ (Fig. 15) to get the same form factor $P(q)$ and the same structure factor $Q(q)$ in all samples and (ii) to adjust the SLD of the continuous phase to the one of the hydrogenated objects $\left(k_{H}=0\right)$, so that the scattered intensity becomes:

$$
I(q)=k_{D}^{2} S_{D D}(q)
$$




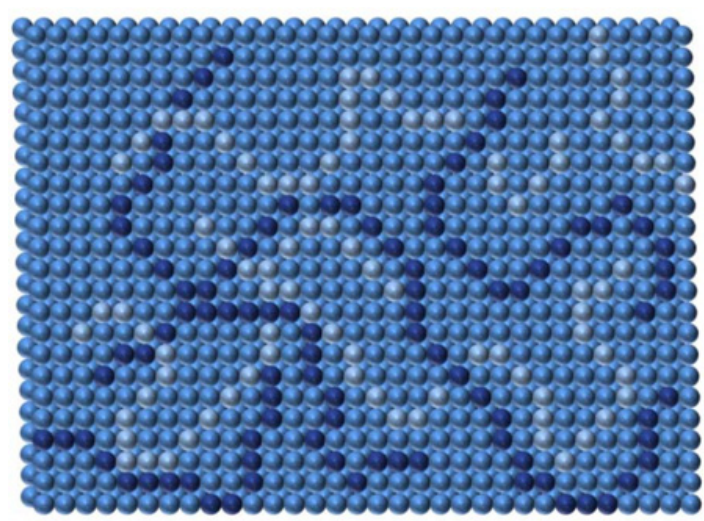

Figure 16. Principle of Zero Average contrast. A Mixture of deuterated and hydrogenated objects is made in a continuous phase of SLD $\rho_{1}$ with a content in deuterated object $x_{D}$ and $\rho_{H}<\rho_{1}<\rho_{D}$. The ZAC conditions are achieved for $\left(1-x_{D}\right)\left(\rho_{H}-\rho_{1}\right)+x_{D}\left(\rho_{D}-\rho_{1}\right)=0$. In such conditions only the form factor is visible.

It comes that:

$$
\frac{I(q)}{k_{D}^{2} x_{D} n N^{2}}=P(q)+x_{D} n Q(q)
$$

The different measurements allow to make the linear interpolation to get $P(q)$ (for $x_{D} \rightarrow 0$ ) and $Q(q$ ) from the slope. It is rather robust since the scattering of structure factor progressively vanishes when decreasing $x_{D}$. If an other kind of object is added within the mixture that has the same SLD as the hydrogenated object $k_{H}$ (one has to be lucky...), the method stay valid since all the contributions from the second kind of object vanished. This has been for example applied to the determination of the conformation of a polyelectrolyte chain within a protein/polyelectrolyte complex (33). Please however note that it is difficult to achieve experimentally with a good accuracy since the linear interpolation has to be done for every $q$. Measurements with very low errors bars are imperative, and thus time consuming.

The second method is very elegant and is called the Zero Averaged Contrast method. The only requirement for such method is that the SLD of the continuous phase must lie between the one of the hydrogenated object and the one of deuterated object.

Let us write the whole scattered intensity (Eqs. (18) and (25)) by separating the form factor and the structure factor:

$$
\frac{I(q)}{n N^{2}}=\left(\left(1-x_{D}\right) k_{H}^{2}+x_{D} k_{D}^{2}\right) P(q, n)+n\left(\left(1-x_{D}\right) k_{H}+x_{D} k_{D}\right)^{2} Q(q, n)
$$

The structure factor completely vanishes when the SLD of the continuous phase is adjusted in a way so that $\left(1-x_{D}\right) k_{H}+x_{D} k_{D}=0$ (zero contrast conditions), because all the amplitude terms of the structure exactly compensate. It is difficult to make a graphic representation of the principle (yet it is represented in Fig. 16). Since there are two levers to play on the ZAC conditions $\left(k_{H}\right.$ and $\left.x_{D}\right)$ they can be achieved for every $k_{H}$ and allow thus to match a second specie within the mixture (34). For $x_{D}=0.5$, $k_{H}=-k_{D}$ and $I(q)=n N^{2} k_{H}^{2} P(q)=n N^{2} k_{D}^{2} P(q)$.

The main advantage of the method is that is requires only a single measurement, contrary to the interpolation to zero concentration method. Nevertheless, there is no possibility to check experimentally that the extinction of $Q(q, n)$ is effective. The SLD have to be perfectly determined beforehand. 


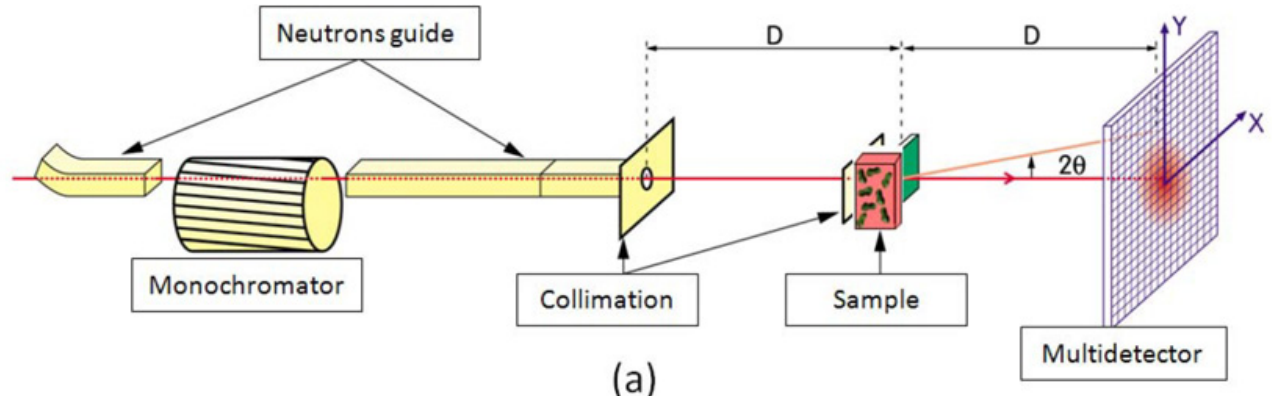

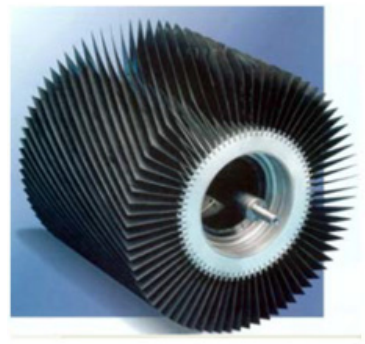

(b)

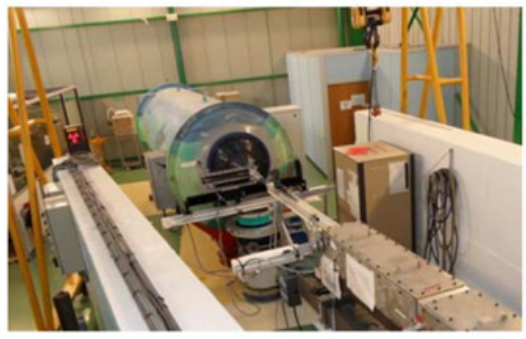

(c)

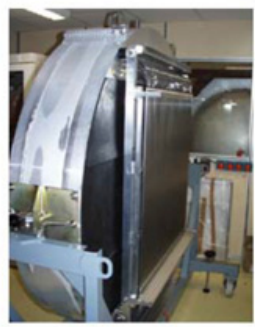

(d)

Figure 17. (a) Layout of a SANS spectrometer; (b) Picture of a velocity selector; (c) Picture of the PAXE spectrometer at LLB (now dismantled); (d) Picture of the PAXY detector.

\section{Experimental aspects}

This second part is devoted to the experimental aspects of SANS to help users optimizing their experiments.

\subsection{Spectrometers}

There are two of ways of performing SANS measurements. Since q depends both on $\theta$ and $\lambda$, it is first possible to work at fixed $\lambda$ and to record the scattered intensity as function of $\theta$. This method is the most common way of measuring and is perfectly suited for continuous sources (reactors) and will be described hereafter. The other way which is specific of neutrons is to work in time of flight mode to use the white polychromatic beam to vary $\lambda$ and to work at fixed $\theta$. Briefly, the idea is to create a pulse of polychromatic neutrons at a given position prior to the sample and to measure the time $t$ taken by the neutron to reach the detector located at the distance $\mathrm{L}$ from this point. As it has a mass, the neutron moves at a finite speed, linked to its wavelength via the De Broglie relation $v=\mathrm{h} / m \lambda$ where $\mathrm{h}$ is Planck constant and $m$ the neutron mass, which ranges from a few $100 \mathrm{~m} / \mathrm{s}$ to a few $1000 \mathrm{~m} / \mathrm{s}$ for cold neutrons. The neutrons of different wavelengths are then separated through their speed, the fastest neutrons moving faster than the slower ones. It method enables to work with a good resolution (35) (36). It is obviously suited to pulsed sources (spallation sources) and is lead to develop since all new modern bright sources are spallation sources.

\subsubsection{Layout of a spectrometer}

The classical layout of a spectrometer is shown in Fig. 17a and is basically made of a monochromator, a collimator and a detector. 
The monochromator is classically made of a mechanical velocity selector (see picture of Fig. 17b), instead of using a crystal or mirrors that have a good resolution but provides only a poor neutron flux. It enables to select the neutrons that have a given speed $\mathrm{v}$ from the incident continuous beam outcoming from the reactor and to absorb all the others ones. The selection is achieved by a cylindrical rotor made of a material that absorb neutrons (for instance the black $\mathrm{B}_{4} \mathrm{C}$ in the picture of Fig. 17b) containing a helix allowing the passage of neutrons. The rotation axis is parallel to the neutron beam and the direction of rotation opposite to the helix one. Now, imagine that you are travelling on a neutron that has the speed to be selected. You will see a window opens in front of you all along your travel within the velocity selector. On the contrary, if your travel on a neutron that is too fast or to slow, you will be absorbed on the edges of the window. The velocity selector has two advantages: $(i)$ it let passes a large number of neutrons detrimental of the resolution (see part 3.1.2) and (ii) the speed of neutrons to be selected, and therefore its $\lambda$, can be easily changed by modifying the speed of rotation of the selector (from $\sim 800$ to $5000 \mathrm{rpm})$.

The neutrons beam has to be collimated because it is very divergent at the exit of the neutron guide that make the neutrons travel from the reactor to the spectrometer. The principle of the collimator is very simple because it is hardly impossible to make converging the beam by lenses. The neutron refractive index of material is indeed always $1 \pm 10^{-3}$, that give very long focal distances (see the neutron reflectivity course in this issue). It is then simply made of two circular diaphragms made of neutrons absorbent material placed at the exit of the guide and in front of the sample, of respective radius $r_{1}$ and $r_{2}$ (pinhole collimation), $r_{1}$ being larger than $r_{2}$ and the surface of second diaphragm being smaller than the one of the sample. In order to keep reasonable fluxes, the typical values of the diaphragms values vary from $5 \mathrm{~mm}$ to several $\mathrm{cm}$. The collimation length $\mathrm{D}_{c}$ is usually of the order of the sample-detector distance D. The position of the first diaphragm is usually tunable to adjust $\mathrm{D}_{c}$ to $\mathrm{D}$. A removable neutron guide is then placed within the velocity selector and such first diaphragm to optimize the neutrons flux. Since the attenuation of beam by air is not negligible (around 10\% per meter) due to scattering or absorption (nitrogen, water), the collimator is under vacuum.

The detector is usually a 2D detector whose size ranges from $64 \mathrm{~cm} \times 64 \mathrm{~cm}$ to $1 \mathrm{mx} 1 \mathrm{~m}$. It is made of gases that absorb neutrons $\left({ }^{3} \mathrm{He}, \mathrm{BF}_{3} \ldots\right)$ to emit a $\gamma$ that is detected by photoelectric effect. The detector is usually an XY 2D grid with squared pixels whose size are $0.5 \mathrm{~cm} \times 0.5 \mathrm{~cm}$ or $1 \mathrm{~cm} \times 1 \mathrm{~cm}$ (there are also annular detectors such as on PACE at LLB). In order to absorb the direct beam, id est the neutrons that are not scattered by the sample, a beam catcher of radius $3-4 \mathrm{~cm}$ is set in front of the detector at the place of the direct beam. This beam-catcher is removable in order to measure the incident flux of the beam or the transmission of the samples. As for the collimator, the detector has to be placed within a tank under vacuum to avoid the attenuation by air. It is usually mounted on a rail within the tank to allow a continuous variation of the sample-detector distance.

To conclude this part, let us make a remark on the dimensions of a SANS machine. The aim of the purpose is to work at the smallest possible q's. Since it is not possible to play at will on the wavelength (in practice, the fluxes for the neutrons of wavelengths larger than $12 \AA$ are too poor on cold sources to be used), the only possibility is to measure at very small angles, which give its name to the technique. On the same time, one wants to work with large apertures within the collimator to keep a reasonable incoming flux. The only way to measure at small angles with a correct q definition is then to build very large machines. The sample-detector lies then usually between $5 \mathrm{~m}$ and $20 \mathrm{~m}$ ! The overall length of the machine has to be doubled owing to the collimation. The sample-distance of the D11 spectrometer at ILL reaches around $40 \mathrm{~m}$, and its total length is more than $80 \mathrm{~m} \mathrm{(37) \ldots .}$

\subsubsection{Experimental resolution of a spectrometer}

Please first keep in mind that a SANS experimental is always an experiment with a poor experimental resolution. There are three main contributions to this resolution coming from the velocity 
selector, the collimator and from the detector, this last contribution being important and not to be forgotten. In the following I have only reported the most important formula. More information and calculations can be found in reference (35) from D. Lairez.

The $\Delta \lambda / \lambda$ due to the velocity selector ranges typically from $5 \%$ to $10 \%$ and depends on the angle between the velocity selector axis and the beam (this angle that fixes the $\lambda$ versus speed can be slightly changed to either increase flux or resolution). It does not depend on the wavelength and can be approximated by a Gaussian law $\mathrm{f}\left(\lambda, \lambda_{0}, \Delta \lambda\right)$ centered around a mean wavelength $\lambda_{0}$ (see below) and of standard deviation $\Delta \lambda$. Please note that the distribution of wavevectors $k \mathrm{f}\left(k, k_{0}\right)$ which is the relevant parameter for $\mathrm{q}$ is no longer Gaussian and is asymmetric since with $k=2 \pi / \lambda$.

The angular contribution from the collimator $\Delta \theta_{c o l}$ depends on the respective radius $r_{1}$ and $r_{2}$ of the diaphragms, on the collimation length $\mathrm{D}_{c}$ and from the sample detector $\mathrm{D}$ :

$$
\Delta \theta_{c o l}=\frac{r_{1}}{D_{c}}+r_{2}\left(\frac{1}{D}+\frac{1}{D_{C}}\right)
$$

It can be shown that the flux can be optimized if $r_{1} / r_{2}=\left(D+D_{c}\right) / D$. In this case the previous equation reduces to $\Delta \theta_{\text {col }}=2 \mathrm{r}_{1} / \mathrm{D}_{c}$. The resolution function due to the collimation can be approximated by either a triangle function or a Gaussian function.

The third contribution comes from the factor that the detection of the position is not punctual because the cells from the detector have a finite size. This may have a huge impact, especially for the closest cells from the beamstop. On PACE (LLB), the beamstop radius is $3 \mathrm{~cm}$ large and the pixel size is $1 \mathrm{~cm}$ large: the first measured point is then integrated from 3 to $4 \mathrm{~cm}$ to the center... The resolution function from the detector is a crenel function for annular detector and a trapezoidal function for a 2D-grid detector because the square pixels overlap when the signal is radially averaged.

The angular resolution function of the spectrometer depends thus both on collimation and detector and is then the convolution of the Gaussian function describing the collimation with this crenel or trapezoidal function.

Since $\mathrm{q}$ can be approximated by $\mathrm{q}=k \theta$ at small angles $(\sin \theta \sim \theta)$, the overall resolution function of the spectrometer $\mathrm{R}\left(\mathrm{q}, \mathrm{q}_{0}\right)$ comes from the convolution of the wavevector resolution function and the angular resolution function. It cannot be easily exactly analytically calculated and, rigorously, varies from one cell of the detector to another, id est it is $\mathrm{q}_{0}$-dependent. In practice, for a given configuration, the resolution will be dominated by the angular resolution at small angles and by the wavevector resolution for the largest angles. Finally since both are Gaussian functions, the overall function can be well approximated by:

$$
R\left(q, Q_{0}\right)=\frac{1}{\sqrt{2 \pi} \Delta q} e^{-\frac{\left(q-q_{0}\right)^{2}}{2 \Delta q^{2}}}
$$

with

$$
\Delta q=\left(q_{0}^{2}\left(\frac{\Delta k}{k_{0}}\right)^{2}+k_{0}^{2} \Delta \theta^{2}\right)^{1 / 2}
$$

Please note that this function is asymmetric. In practice, the ratio $\Delta k / k$ is equal to $\Delta \lambda / \lambda$ and is thus q-independent. The width of the angular resolution is also q-independent and can be described by:

$$
\Delta \theta=\left(\frac{\Delta \theta_{\text {col }}^{2}}{2}+\frac{\Delta \theta_{\text {det }}^{3}}{3}\right)^{1 / 2}
$$

where $\Delta \theta_{\text {det }}=\Delta \mathrm{R} / 2 \mathrm{D}$ with $\Delta \mathrm{R}$ the radial dimension of the detector. 

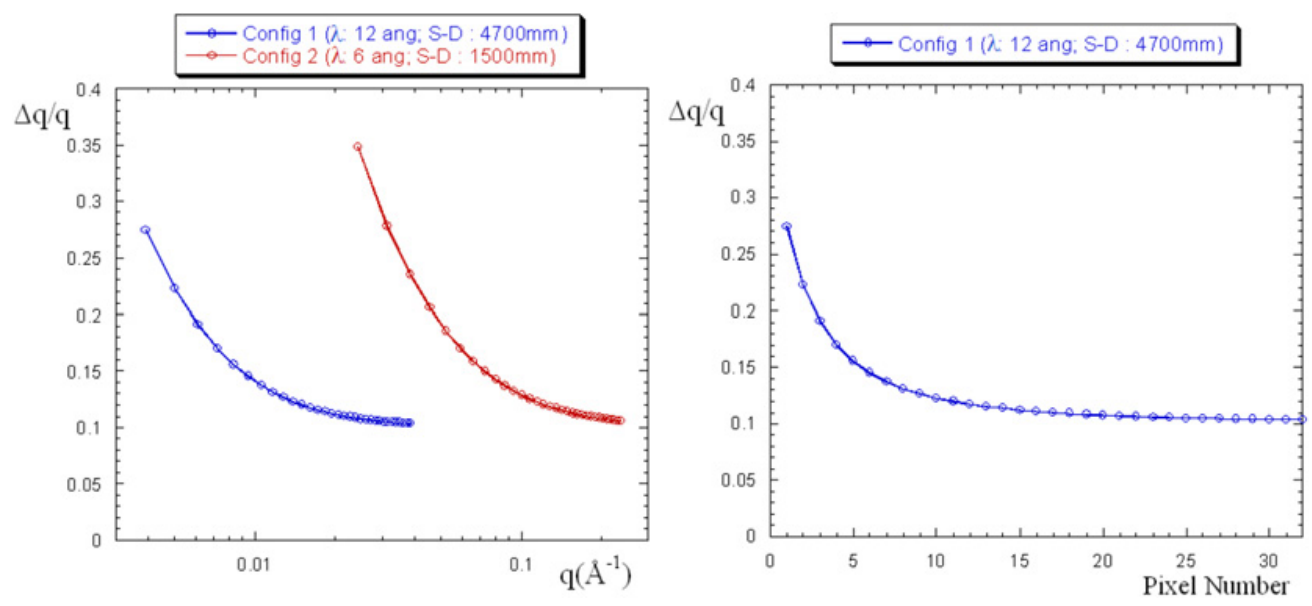

Figure 18. Resolution of a spectrometer for each measurement point. Calculation based on the PACE spectrometer $(\Delta \lambda / \lambda \sim 10 \%$, pixel size: $10 \mathrm{~mm}$, first pixel of measurement located at $3 \mathrm{~mm}$ from center detector size). (a) resolution for two configurations routinely used: configuration 1: $\lambda=12 \AA$; sample-Detector distance D: $4700 \mathrm{~mm}$, $r_{1}: 12 \mathrm{~mm}$; configuration 2: $\lambda=6 \AA$; sample-Detector distance D: $1500 \mathrm{~mm}, r_{1}: 16 \mathrm{~mm}$. (b) Resolution of Fig. 1 where the pixel position is represented instead of q.

Figure 18 shows the relative resolution $\Delta \mathrm{q} / \mathrm{q}$ as a function of $\mathrm{q}$ for the PACE spectrometer at LLB. Please note that this relative resolution it very degraded for the smallest q.

\subsubsection{Choosing its configuration for an experiment}

Since the allowed beamtime is always limited, it is of great importance to choose properly the configurations used for the experiment. Each configuration is defined by a couple of $\left(\lambda, D_{\text {sample-detector }}\right.$ ) enabling to measure over a range $q_{\min }-q_{\max }$ that covers generally one decade. $\mathrm{q}_{\text {min }}$ is set by the $\theta_{\min }$ that is possible to reach next the edge of the beamstop and $\mathrm{q}_{\max }$ by the diameter of the detector. When the scattering of the sample is isotropic and that one uses an XY 2-D detector, it is possible to shift the direct beam far from the center of the detector to increase the q-range probed. Thus, depending on the aim of the experiment, 1, 2 or 3 different configurations will be used to make measurements on a greater or lesser q-range. Although this may be tempting to choose two adjacent configurations that have a very small recovery to increase as much as possible the q-range, this is in practice a mistake because some problems may appear when merging the scattering curves for two different configurations. Indeed the experimental resolution is very different for the cells of the detector close to the beamstop than for the ones at the border of the detector (see 3.1.2).

It is important to have a raw idea of the typical sizes to be probed in direct space to choose the right number of configurations to be used. Discussions with the local contact can be helpful... When no information is available on the samples, one works in a "blind" way and one has to probe the largest possible q-range with several configurations. A proper way of working to save beamtime is to make in a first time a "snapshot" of the sample scattering by a 1 minute -measurement and to adjust in the second time the dedicated time of measurement for each configuration to obtain a good signal-to-noise ratio.

It is also important to optimize the configuration for a given q-range to be probed. It is indeed possible to probe the same q-range with an infinite of couples $\left(\lambda, \mathrm{D}_{\text {sample-detector }}\right)$ but in practice the incoming neutron flux $\mathbf{J}$ will vary from one configuration to another. Three parameters have an impact on such flux: $(i)$ the variation of $\mathbf{J}$ as a function of the geometric parameters that are the angle of measurement $\theta$ and collimation, (ii) the variation of $\mathrm{J}$ with the wavelength $\mathrm{J}_{0}(\lambda)$ and (iii) the efficiency 
of the detector as function of wavelength $\varepsilon(\lambda)$. It immediately appears that taking away the detector from the sample strongly decreases $J$ because the solid angle is decreased $\left(\propto 1 / D_{\text {sample-detector }}{ }^{2}\right)$ and because the collimation must be more accurate. It could be demonstrated that the flux corresponding to the optimized collimation varies like $\mathrm{J}_{0} \theta^{4}(36)$. $\mathrm{J}_{0}(\lambda)$ has a Maxwellian shape because it corresponds to the thermalization of a perfect gas of neutrons at the temperature of the cold source moderator and follows the Maxwell-Boltzmann statistics $\mathrm{P}(\lambda)=\exp \left(-\left(\lambda_{\text {therm }} / \lambda\right)^{2}\right) \times \lambda^{-4}$; where $\lambda_{\text {therm }}$ is the thermal length. The efficiency of the detector increases with the wavelength $\left(\varepsilon(\lambda)=1-\mathrm{e}^{-\lambda / \lambda \mathrm{c}}\right.$ where $\lambda_{\mathrm{c}}$ is a critical value that is dependent on the detector used). Altogether, the combination of the effects of $\mathrm{J}_{0}(\lambda)$ and $\varepsilon(\lambda)$ provides an usable flux $\mathrm{J}_{\text {use }}(\lambda)$ that an asymmetric shape, a maximum $\lambda_{\max }$ (around $3-4 \AA$ on the SANS machines at LLB) and a raw decay like $\lambda^{-4}$ for large $\mathrm{q}$ above $\lambda_{\max }$ (the decay to the shortest wavelengths below is very sharp, anyway the short wavelength are not useful for SANS) (36). For a given targeted q-range corresponding to one configuration, there is always a set of $\left(\lambda, \mathrm{D}_{\text {sample-detector }}\right)$ that optimizes the flux. It is described in (36) how to obtain it. It is in principle chosen after being discussed with the local contact. When only one configuration is necessary for the experiment, such optimized couple has obviously to be chosen. When several configurations are required, the optimized configurations have to be chosen if the intrinsic scattering from the sample is poor. However it obliges to change the wavelength for each configuration, which may give raise to some difficulties when merging data since the transmission depends on the wavelength (see part on data reduction later). When scattering from sample is large, a convenient way to work is then then to choose a constant wavelength for all configurations, close to the $\lambda_{\max }$ of $\mathrm{P}(\lambda)$, and to only change the distance. This last method is generally used for SANS machines that enable a large range of sample-to-detector distances $(\sim 15-20 \mathrm{~m})$.

From a general point of view, whatever the configuration chosen, it appears that the flux always very strongly decreases when going towards low q's because of the combined effects of wavelength $\left(\sim \lambda^{-4}\right)$ and angle $\left(\sim \theta^{4}\right)$. Although the scattered intensity is generally much higher at small $\mathrm{q}$ than at low q (see the form factors on part 2), it is in practice always much more difficult to make measurements at small $q$ than at large $q$ because the high intrinsic scattered intensity at small q does not compensate the very poor incoming neutron flux for such q-range. The counting rates are then much larger for the smallest q (generally 15 min to few hours for $10^{-3} \AA^{-1}<\mathrm{q}<10^{-2} \AA^{-1}$ ) than for the largest q (generally $5 \mathrm{~min}$ to $15 \mathrm{~min}$ for $510^{-2} \AA^{-1}<\mathrm{q}<510^{-1} \AA^{-1}$ ) to get a good signal-to-noise ratio. The only case where the effects almost compensate themselves concerns the sample that scatters like $\mathrm{q}^{-4}$ following a Porod law. Please note that this is the inverse case than for reflectivity for which the measurements at the smallest $q$ are always easier.

\subsection{Samples and environments}

\subsubsection{Samples}

In this section I provide some considerations on the samples for achieving a successful experiment. The objective is usually to obtain an experimental curve with a good signal to noise ratio and small error bars, which is easily achieved if the scattering of the sample is huge. Please however remember that it is not necessary a good new when your sample scatters a lot, even it generally makes your local contact happy, because it is often a signature of aggregation... A sample that does not scatter is indeed sometimes a good result (32)! It is also worth to recall the experimental limits of the experiment in term of intensities. A priori there is not upper limit for the scattered intensity because it will be easily measured. However one must be cautious with a sample that strongly scatters because it can be the signature of multiple scattering (see below). There is on the contrary a practical bottom limit for the measurement. It is indeed very difficult to go down to $10^{-2} \mathrm{~cm}^{-1}$ and challenging to go down to $10^{-3} \mathrm{~cm}^{-1}$, unless counting for days. The coherent scattered intensity of the sample must thus be optimized. 


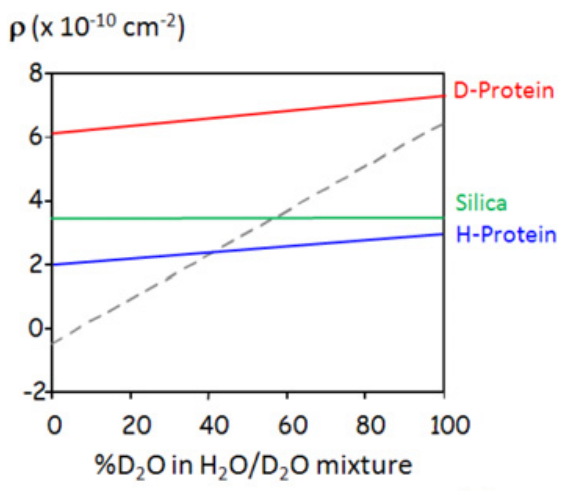

(a)

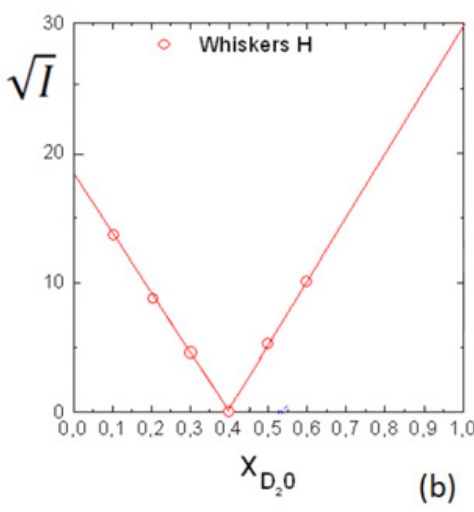

(b)

Figure 19. Evolution of the SLD of proteins that contain some hydrogen in a $\mathrm{H}_{2} \mathrm{O} / \mathrm{D}_{2} \mathrm{O}$ mixture. Silica that does not contain any labile hydrogen is shown as example. The dotted line is the SLD of water. The intersection between this dotted line and the continuous lines that give SLD of objects enable to find the matching point. Adapted from (38). (b) Principle of contrast variation adapted to the determination of the SLD of hydrogenated cellulose whiskers (unpublished results).

Let us consider the simple and very representative case of a system made of objects in a continuous media:

$$
I(q)\left(\mathrm{cm}^{-1}\right)=\Phi\left(\rho_{o b j}-\rho_{\text {media }}\right)^{2} V_{o b j} K(q)
$$

where $K(q)$ is the scattering function that accounts for the structure factor $\mathrm{S}(\mathrm{q})$ and form factor $\mathrm{P}(\mathrm{q})$. Both the volume fraction Фand contrast term $\left(\rho_{\mathbf{o b j}}-\rho_{\text {media }}\right)^{2}$ are pre-factors that can be evaluated prior to the experiment and that have to be optimized. If the raw estimate of $V_{o b j}$ can be done, it is possible to calculate $\Phi\left(\rho_{o b j}-\rho_{\text {media }}\right)^{2} V_{o b j}$. It will provide a very good estimate of $\mathrm{I}(\mathrm{q})_{q \rightarrow 0}$ and will allow to determine if the experiment is feasible.

When the continuous media is a solvent, as most of time in soft matter or biology, the contrast term can be easily tuned by deuteration. Since the intensity is proportional to the square of the difference of the SLD between components in this contrast term, it is often the key to the success of an experiment. The calculation of SLD is very simple for crystals or solvents ${ }^{\ddagger \ddagger}$ but may give rise to some difficulties for glasses or polymers when the exact value of specific volume of the molecule is not determined with certainty. Please take care of the objects that contain labile protons and are immersed in a $\mathrm{H}_{2} \mathrm{O} / \mathrm{D}_{2} \mathrm{O}$ mixture, because there is an exchange between the protons of objects and those of water. In this case, the SLD of the objects depends on the $\mathrm{H}_{2} \mathrm{O} / \mathrm{D}_{2} \mathrm{O}$ ratio and increases with the content of $\mathrm{D}_{2} \mathrm{O}$ (see Fig. 19a adapted from B. Jacrot (38)).

If the SLD is unknown, it can be measured by SANS by the contrast variation method based on the last Eq. (70). Briefly, one makes several samples that are exactly similar in water (or in an organic solvent), except the $\mathrm{H}_{2} \mathrm{O} / \mathrm{D}_{2} \mathrm{O}$ ratio $x$ that is varied from 0 to 1 . $\sqrt{ }$ I versus $x$ is then plotted (for either a given q or for the integral of the scattering curve, after the proper subtraction of the incoherent scattering that strongly vary with $x$ ). It is linear and its interpolation to $\sqrt{ } \mathrm{I}=0$ provides the $x_{\text {match }}$ that matches the

\footnotetext{
$\sharp$ An example of calculation of scattering length density: the case of water. This density is obtained through $\rho=\left(\Sigma_{i} \mathrm{~b}_{i}\right) / \mathrm{V}$ where the $\mathrm{b}_{i}$ correspond to the atoms constitutive of the molecule (Table 1) and $\mathrm{V}$ the volume of the molecule. Since the mass molar of $\mathrm{H}_{2} \mathrm{O}$ is $18 \mathrm{~g} / \mathrm{mol}$ and the density of $\mathrm{H}_{2} \mathrm{O} 1 \mathrm{~g} / \mathrm{cm}^{3}$, the volume of a molecule of water is $18 / 6.0210^{23}=2.9910^{-23} \mathrm{~cm}^{3} \mathrm{per}$ molecule. The volume of a molecule of $\mathrm{D}_{2} \mathrm{O}$ is similar. On gets:

$\rho\left(\mathrm{H}_{2} 0\right)=(-0.374 * 2+0.58) \cdot 10^{-12} / 2.9910^{-23}=-0.5610^{10} \mathrm{~cm}^{-2}\left(-0.5610^{-6} \AA^{-2}\right)$ $\rho\left(\mathrm{D}_{2} 0\right)=(0.667 * 2+0.58) \cdot 10^{-12} / 2.9910^{-23}=6.3810^{10} \mathrm{~cm}^{-2}\left(6.3810-6 \AA^{-2}\right)$.
} 
objects and therefore their SLD (Fig. 19b). The method is not limited to colloids or nanoparticles and works very well for porous materials soaked in water. It is also possible to dilute a sample initially in $\mathrm{H}_{2} \mathrm{O}$ by $\mathrm{D}_{2} \mathrm{O}$ in order to vary $x$, or the inverse, and to plot $\sqrt{ } \mathrm{I} / \Phi$ versus $x$. It must however be checked in this last case that the dilution does not modify neither $\mathrm{P}(\mathrm{q})$ nor $\mathrm{S}(\mathrm{q})$ because the method is based on the hypothesis that $\Phi K(q)$ stay constant from one $x$ to another, any change of this latter would fail the method.

If it is important to have a sufficient coherent scattering, it is also crucial to avoid a too intense coherent scattering to avoid multiple scattering, id est the probability for a neutron to be scattered more than once by the sample. The formalism presented in part 2 supposes indeed that a neutron is scattered only once by the sample because it is impossible to determine the respective angles of scattering within the sample of a neutron that exit the sample after being scattered several times. Since the relevant scattered intensity per unit volume $\mathrm{I}(\mathrm{q})\left(\mathrm{cm}^{-1}\right)$ is intensive, an easy way to eliminate the multiple scattering is to reduce the thickness of the sample because the overall probability for a neutron to be scattered is proportional to the optical path. Such reduction of the thickness is not a problem to obtain a good signal-to-noise ratio for the signal because samples that may show multiple scattering have indeed an intrinsic huge scattering. An other way to reduce multiple scattering is to reduce the objects concentration in the sample. This is however not recommended because it may change the structure factor obviously and even sometimes the form factor of the scattering objects.

It is obviously not possible to assess a priori if multiple scattering occurs in a sample. There are however some experimental signs of it, like a very sharp and intense scattering at low q or a strong decrease of the transmission. If doubt exists, the possible presence of multiple scattering can be checked. For such a purpose, several measurements of the sample have to be done with different thicknesses. Given that $\mathrm{I}(\mathrm{q})\left(\mathrm{cm}^{-1}\right)$ is independent from the thickness after data reduction (see next part) in absence of multiple scattering, any change of the scattering spectra from one thickness to another would arise from multiple scattering effects. Such method allows to determine the optimal thickness for measurements: this is the threshold thickness below which the scattering becomes constant.

The last important thing to consider is the incoherent scattering (see part 2.2). As is gives no structural information (the probability for a neutron to be scattered by an incoherent process by an atom has a constant probability over $4 \pi$ independently from its positions to the other atoms), it is a background in SANS and has to be reduced as much as possible. Besides, incoherent scattering strongly attenuates the beam (see next part). The incoherent cross-section is very important for ${ }^{1} \mathrm{H}$ (80.26 barns) and rather high for deuterium $\mathrm{D}\left({ }^{2} \mathrm{H}\right)$ (2.05 barns). Apart from hydrogen, the other atoms from periodic table that have a huge incoherent scattering are rare (for instance vanadium). The incoherent scattering comes then essentially for the content in hydrogen or deuterium within the sample. Since it however forty times less for $\mathrm{D}$ than for $\mathrm{H}$, the hydrogen has to be replaced as much as possible by deuterium when possible. In case of a system of objects dispersed in a solvent, the common way is to use a fully deuterated solvent. Please note that this is not a rule. The optimized conditions have to consider both the coherent contrast term $\left(\rho_{o b j}-\rho_{\text {solv }}\right)^{2}$ and the incoherent scattering (Fig. 20). If the SLD of the objects in close to the SLD of the deuterated solvent, measurements in hydrogenated solvent are preferable. Besides, in contrast-variation experiment, the ratio $\mathrm{H} / \mathrm{D}$ content is fixed by the conditions of coherent contrast independently from the incoherent scattering conditions (Fig. 12).

\subsubsection{Sample thickness}

Ideally, the dimensions of the sample should be optimized for the experiment. The surface of sample illuminated by the beam must be slightly larger than the surface of the exit diaphragm of the collimator, id est a circle with a radius lying between $5 \mathrm{~mm}$ and $10 \mathrm{~mm}$. If the expected scattering of the sample is very weak, this diaphragm can be enlarged detrimental of the resolution and the surface of sample has to be enlarged accordingly. 

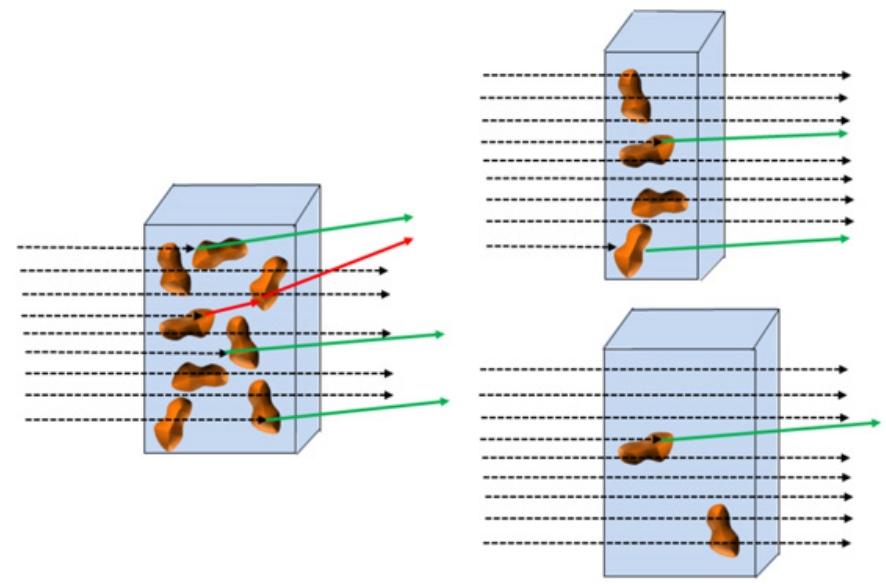

(a)

(b)

Figure 20. Multiple scattering. (a) Principle: Owing to huge concentration and/or large differential scattering crosssection, the neutron is scattered twice (schematized in red). (b) Strategies to avoid multiple scattering: reduction of the thickness of sample (top panel); decrease of concentration (bottom panel). This last strategy is hazardous as it may changes physical properties of sample.
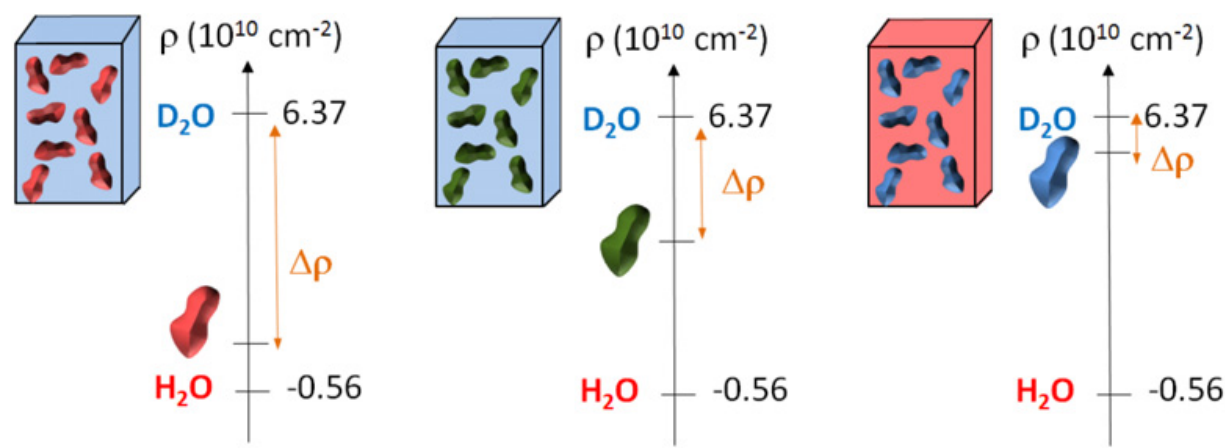

Figure 21. Choice of isotopic composition of a solvent. Both coherent scattering and incoherent scattering have to be considered: usually the choice of deuterated solvent has to be preferred to reduce incoherent scattering except if the SLD of the object is close to the SLD of deuterated solvent.

The concern of the users is generally the optimization of the thickness of the sample $l$. The raw scattered intensity recorded on the detector (in neutrons/sec) varies like $\propto l \exp (-\mu l$ ) since the proportional increase of scattered intensity with the optical path is counterbalanced by the attenuation of the beam by absorption and incoherent scattering, similarly as for the Beer-Lambert law (see part 3.3.1 below). The scattering versus thickness can be then optimized since it passes by a maximum for $l=$ $1 / \mu$ where $\mu$ is the attenuation factor, or equivalently to have a transmission of $\sim 0.37$ (1/e). However one should take care of multiple scattering. In practice, since the transmission is generally essentially dependent on the incoherent scattering, this is the content in hydrogen or deuterium that prompt to choose the thickness of the sample. For example, the transmission by a neutron beam at $\lambda=6 \AA$ is only 0.5 through $1 \mathrm{~mm}$ of $\mathrm{H}_{2} \mathrm{O}$ and 0.8 through $2 \mathrm{~mm}$ of $\mathrm{D}_{2} \mathrm{O}$. The thickness of samples that contain a huge fraction of hydrogen must be of the order of $1 \mathrm{~mm}$ and those containing mainly deuterium of the order of $2 \mathrm{~mm}$, or up to $5 \mathrm{~mm}$ is the content in hydrogen is negligible. 


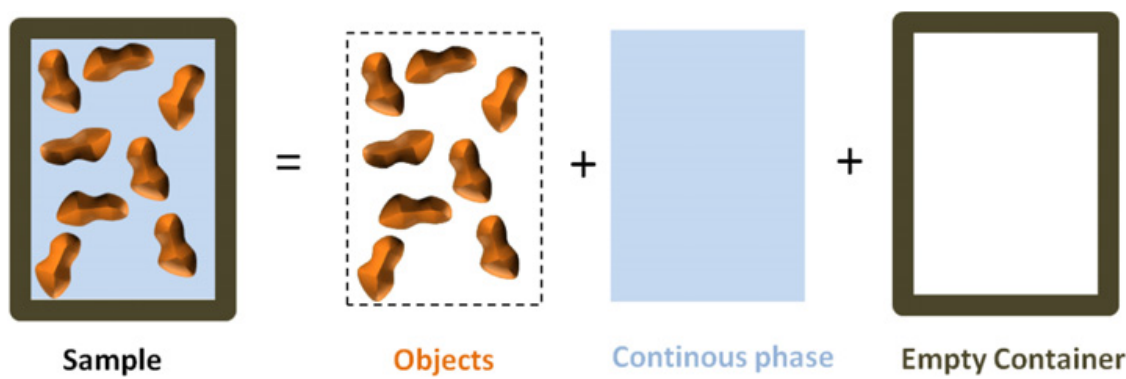

Figure 22. In order to obtain $\mathrm{I}_{\mathrm{obj}}(\mathrm{q})\left(\mathrm{cm}^{-1}\right)$, both the contributions of the empty container and the continuous phase have to be subtracted.

Finally, the surface of illumination ranges between $0.8 \mathrm{~cm}^{2}$ and $3 \mathrm{~cm}^{2}$ and the thickness ranges between 0.1 and $0.5 \mathrm{~cm}$. The volume of sample required for an experiment ranges typically between $0.1 \mathrm{~cm}^{3}$ and $1 \mathrm{~cm}^{3}$.

\subsubsection{Sample environments}

Thanks to the poor absorption of neutrons by matter and in particular by some metallic elements, it is easy to design various samples environments for in situ experiments: tensile machine, rheometer, magnets, cryomagnets, pressure cells, ovens, temperature controllers, etc.. It is advised to contact people from facilities prior to experiments to check that the equipment can be used with the kind of sample to study. It is also of course possible to design its own sample environment for specific experiments.

\subsection{Standard measurements and data reduction}

This section recalls all the measurements that have to be done in addition to the sample for a proper treatment and their respective incidence on the signal.

\subsubsection{Subtraction of parasitic contributions}

There are several contributions to the scattering that are independent of the sample and that have to be subtracted from the raw signal of the sample from a proper data treatment: background of the experiment, contribution for the direct beam and contribution from the empty container if the sample is placed in such container (hellma cell for liquids, dedicated sample environment such as a pressure cell, etc.) (Fig. 22).

The background from the experiment comes from the neutrons that do not come from the beam, and therefore from the settings and configuration and also from the detector electronics. It is important to value it in the same conditions as for the measurements, all things be equal. This is achieved by placing a neutron absorber material instead of the sample, usually boron carbide $\mathrm{B}_{4} \mathrm{C}$ or cadmium (as often referred in the data reduction programs), and to make a measurement. The contribution from the direct beam comes from the neutrons that are not scattered by the sample but reach the parts of the detector next to the beamstop because the incident beam is divergent. It is simply valued by performing a measurement without sample. The contribution of the container is valued by measurement without samples. Its transmission has also to be measured (see below).

The neutron beam is also attenuated by a lineic attenuation factor $\mu_{\text {sam }}$ within the sample due to either absorption or scattering (incoherent and coherent). In practice in soft matter or biophysics the 
attenuation factor is essentially due to the incoherent scattering of the ${ }^{1} \mathrm{H}$ or ${ }^{2} \mathrm{H}$ contained in the sample. The attenuation on a path $\mathrm{z}$ is then $\exp \left(-\mu_{\text {sam }} \mathrm{z}\right)$. In order to take this attenuation into account, an approximation at "small angles" is generally made that assumes that the travel distance within a sample of thickness $l$ is similar for all the neutrons, whether they are scattered or not and whatever the angle of scattering, because $\cos \cong \theta 1$. The attenuation is then similar for all neutrons and is described by the transmission of the sample $\mathbf{T}=\exp \left(-\mu_{\text {sam }} l\right)$. It is measured by means of a detector placed at zero angle by taking the ratio of the intensities measured respectively with and without the sample. In some cases, the approximation cos $\cong \theta 1$ is no longer valid, for instance when the scattering of the container is important or when the transmission of the sample is weak. There are some additional corrections that have to be made (39) (36) which are implemented in the program for data reduction Pasinet at LLB (40).

When the sample is not placed in a container, only the empty beam has to be subtracted. The attenuation of this latter by the transmission of the sample has to be taken into account. The contribution of the background has to be subtracted directly from the raw signal of sample and direct beam. In next equation the respective intensities measured experimentally on a given position of the detector (in neutrons/sec) are already divided the incident flux on the sample $\Phi_{0}$ (in neutrons/sec) and by the solid $\Delta \Omega$ angle (in $\mathrm{cm}^{2}$ ) and the thickness of the sample $l$ in $\mathrm{cm}$ :

$$
I_{\text {sam_sub }}(q)\left(\mathrm{cm}^{-1}\right)=\frac{I_{\text {sam }}(q)-I_{b g k}(q)}{l T_{\text {sam }}}-\frac{I_{E B}(q)-I_{b g k}(q)}{l}
$$

where $I_{\text {sam_sub }}, I_{s a m}, I_{E B}$ and $I_{b g k}$ refer respectively to the scattering of sample after subtraction, the raw scattering of the sample, the scattering of empty beam and the background measured on the pixel of detector corresponding to q, and $T_{\text {sam }}$ to the transmission of the sample.

When the sample is placed in a container, the scattering from the empty beam is already contained in the container and has not to be measured separately. The subtraction is then:

$$
I_{\text {sam_sub }}(q)\left(\mathrm{cm}^{-1}\right)=\frac{I_{\text {sam }}(q)-I_{b g k}(q)}{l T_{\text {sam }}}-\frac{I_{E C}(q)-I_{b g k}(q)}{l T_{E C}}
$$

where the notations are similar as in previous equations except $I_{E C}$ that refers to the scattering of the empty container without sample and $\mathrm{T}_{E C}$ to its transmission.

\subsubsection{Normalization of the spectra and absolute units}

Since the signal is measured by a $2 \mathrm{D}$ detector, there are additional corrections to make for a correct data reduction.

First, some parts of the detector have to be removed. This happens when there are parasitic reflections from the direct beam (within the collimator or following a bad alignment of the spectrometer) that give rise to a punctual intense beam neutron on the detector or when some parts of the detector are damaged (a pixel or a line for example) (see Fig. 23a). These parasitic reflections or huge defaults are removed by a mask that removes all the undesired parts of the detector. The design of the mask is the first step of the data reduction.

Second, it is necessary to take into account the fact that each pixel for the detector has its own efficiency (Fig. 23b). This comes from two origins: $(i)$ each pixel has its electronic discrimination threshold; ( $i$ ) the efficiency of a pixel depends on the length of the path travelled by a neutron within the gas of the detector that has an angular dependence like $1 / \cos \theta$ because the detector is flat and not spherical. Remark that for a given configuration the efficiency of a pixel is constant and does not depend on the sample. In order to overcome these fluctuations of efficiencies, a reference sample whose signal is perfectly flat over the q-range considered is measured. It can be either a pure incoherent scatterer (vanadium), or light water $\mathrm{H}_{2} \mathrm{O}$ (or an hydrogenated solvent) whose scattering is dominated by incoherent scattering and that has a coherent scattering that is flat on this q range (because for the 


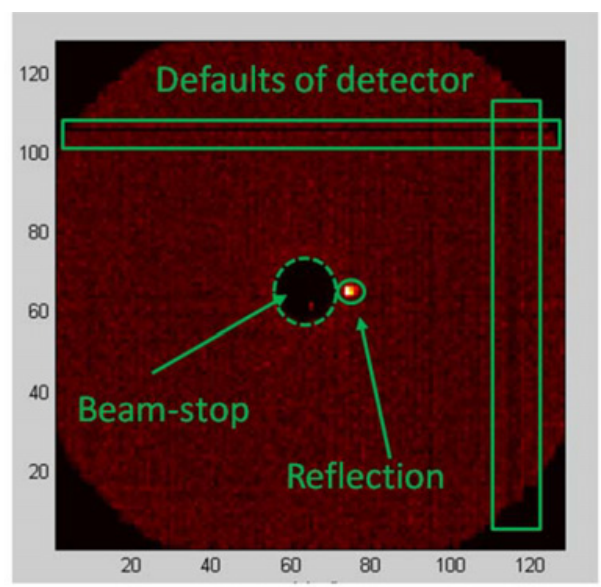

(a)

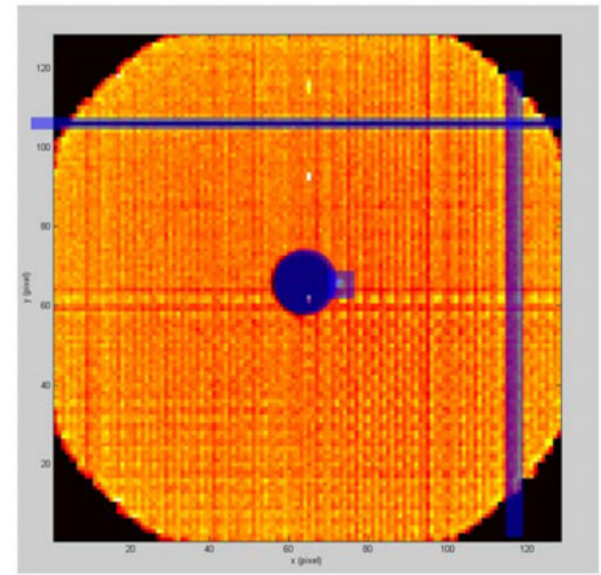

(b)

Figure 23. Corrections to be performed related to the detector. Both panels presents the raw 2-D picture detector of the measurement of a sample made of pure hydrogenated PMMA (PAXY@LLB): (a) The black lines and black columns correspond to some damaged parts of the detectors (fortunately the detector has been replaced in early 2014) and an intense parasitic reflection can be observed close to the beam stop. (b) Once the unusable parts of the detectors have been masked, there remain some fluctuations of intensity from one pixel to another, while the expected scattering should be perfectly flat, sample being a pure incoherent scatterer. This comes from slight differences of efficiency between the pixels. The intensity scale has been changed between the two pictures.

small $\mathrm{q}$ which are measured only the compressibility of water is probed). The scattering of the sample is then divided pixel by pixel (or $q$ by $q$ after radial averaging) by the signal of this reference sample. This renormalization enables to smooth the heterogeneities of the detector. When light water is used, the signal of the empty container of water has to be subtracted from the one of water. Please note that the empty container for water is not necessary the same as those of sample. Finally, in order to achieve subtraction and normalization, the following formula is applied (in case of water):

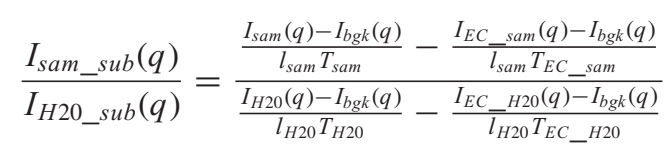

where $I_{\text {sam }}, I_{E C \_s a m p}, I_{b g k}, I_{H 2 O}$ and $I_{E C \_H 2 O}$ refer respectively to the raw scattered intensities of the sample, of empty container for the sample, of the background, of the water in its container and the empty container for water, $l_{\text {sam }}$ and $l_{H 2 O}$ refer to the thicknesses of the sample and water, $T_{\text {samp }}, T_{E C}$ samp $_{\text {sam }}, T_{H 2 O}$ and $T_{E C_{-} H 2 O}$ refer respectively to the transmissions of the sample, of empty container for sample, of the water in its container and the empty container for water.

When the sample or the flat sample (in case of vanadium) are measured without empty containers, the intensities $I_{E C \_s a m p}$, and/or $I_{E C \_H 2 O}$ have to be replaced by the intensity of the empty beam $\mathrm{I}_{E B}$.

If such data reduction strategy allows a proper treatment for all parasitic contributions, it provides only a signal without dimension in the so-called " $\mathrm{H}_{2} \mathrm{O}$ units". The last step is to multiply the whole scattering by the apparent differential scattering cross section of the flat sample $\left.\frac{1}{v} \frac{d \sigma}{d \Omega}\right)_{H 20}$ used for normalization, which is constant over q, to obtain a scattering in absolute units and depends on the spectrometer and configuration (see (36)). This $\left.\frac{1}{v} \frac{d \sigma}{d \Omega}\right)_{H 20}$ is often described as the normalization factor.

$$
\left.\left.\frac{1}{v} \frac{d \sigma}{d \Omega}\right)_{\text {sample }}(q)\left(\mathrm{cm}^{-1}\right)=\frac{I_{\text {sam_sub }}(q)}{I_{H 20 \_s u b}(q)} \frac{1}{v} \frac{d \sigma}{d \Omega}\right)_{H 20}
$$


The normalization factor is often tabulated as function of wavelength on the spectrometers. It could be measured if necessary. The following standards are required: $(i)$ the number of neutrons scattered by the flat sample on the detector of surface $\mathrm{S} \Delta N_{H 2 O}$ (in neutrons/sec); (ii) the number of neutrons scattered by the empty container of the flat sample on the detector of surface $\mathrm{S}$, or the empty beam, $\Delta N_{E C}$ (in neutrons/sec); (iii) the respective transmission of the flat sample and of empty container $\mathrm{T}_{H 20}$ and $\mathrm{T}_{E C_{-} H_{2} O} ;(i \mathrm{v})$ the incoming neutrons flux $\Phi_{0}$ (in neutrons/sec) at the chosen configuration.

$$
\left.\frac{1}{v} \frac{d \sigma}{d \Omega}\right)_{H 20}\left(\mathrm{~cm}^{-1}\right)=\frac{\Delta N_{H 20}-\frac{T_{H 20}}{T_{E C}} \Delta N_{E C}}{\Phi_{0} T_{H 20} \Delta \Omega l_{H 20}}
$$

where $l_{H 2 O}$ is the thickness of the flat sample in $\mathbf{~ c m}$ and $\Delta \Omega$ the solid angle ( $/ \mathrm{D}^{2}$ with $\mathrm{D}$ the sampledetector distance). In practice all these standards are already measured because they are required for the subtraction and normalization steps except the incoming neutrons flux $\Phi_{0}$. $\Phi_{0}$ is the incident empty beam and depends on the parameters listed in part 3.1.3. It is measured without beamstop. Since it is very often very intense, it cannot be directly measured and its intensity has to be attenuated. Indeed, if the neutrons flux reaching the detector is very high, the electronics cannot measure all neutrons because it requires a necessary time to register one neutron, the so- called "dead-time". Dead-time problems do not usually occur when measuring scattering but may be important when measuring transmission. The attenuation is achieved by setting an attenuator within the beam, generally a controlled thickness of PMMA. The attenuation factor has then to be measured. It is valued by measuring the ratio of the scattering of a strong scatterer (usually graphite), in presence of the attenuator, to its non-attenuated scattering.

\subsubsection{Subtraction of solvent and/or incoherent scattering}

Once the data reduction depicted in the previous parts is applied, there are often still some subtractions to achieve that come from contributions of the sample itself. In a two-phase system made of objects in a continuous phase with a volume fraction $\Phi_{\text {objects }}$ such as a solvent, the intensity writes:

$$
I_{\text {sample }}(q)\left(\mathrm{cm}^{-1}\right)=\Phi_{\text {objects }} I_{\text {objects }}(q)\left(\mathrm{cm}^{-1}\right)+\left(1-\Phi_{\text {objects }}\right) I_{\text {solvent }}(q)\left(\mathrm{cm}^{-1}\right)
$$

The solvent scattering $\mathrm{I}_{\text {solvent }}(\mathrm{q})$ has a coherent part (generally weak) and an incoherent part. It has to be measured independently and subtracted from the sample to obtain the desired $\mathrm{I}_{\text {objects }}(\mathrm{q})$ intensity, taking into account the $\left(1-\Phi_{\text {objects }}\right)$ term. It generally reduces to a constant. It is then preferable to subtract such constant from the sample $\mathrm{I}_{\text {sample }}(\mathrm{q})$ rather than subtracting $\mathrm{I}_{\text {solvent }}(\mathrm{q}) q$ by $q$ not to increase the error bars.

If the objects are constituted of atoms whose incoherent scattering is null, as silica nanoparticles for example, the subtraction of solvent is sufficient. Otherwise the incoherent scattering of the objects themselves has to be subtracted. It can be valued from the composition of the objects but this is not always possible and must be estimated by others means.

An approach is to consider that the transmission of the sample is only driven by the incoherent scattering of both the objects and the solvents. A witness sample made of a mixture of deuterated and hydrogenated that has exactly the transmission as the sample is measured. Its scattering is then subtracted from the sample. In practice it is difficult to directly happen on the good H/D ratio and several mixtures are made, the level of scattering to subtract being obtained by interpolation. This method requires obviously transmission measurements with a very good accuracy.

There is another method for the subtraction of the incoherent scattering that has the advantage to be fast and does not require additional measurements known as the "far point subtraction" method. It necessitates that the measurement of the sample has been achieved up to very large q and supposes that one probes on this q-range the form factor of an object whose scattering decay is known. For instance, if one probes bulky objects, the scattering will decay like $\mathrm{q}^{-4}$ at very large $\mathrm{q}$ thanks to the Porod law. 
The idea is then to subtract a constant from the whole scattering to force the $q^{-4}$ scattering at large $q$. It works very well for 3-D objects (nanoparticles, folded proteins...) but has to be used with great caution if one studies objects like polymers. While it appears at first sight not very rigorous, it is very efficient in practice.

To conclude this part let us remark that a proper subtraction of incoherent scattering requires a measurement of the scattering pattern with a very good accuracy at large q because it is in this very range that it raises difficulties. Fortunately this is the q-range for which it is possible to reach a signal with a very good-signal to noise ratio because the incoming flux is important (see 3.1.3). It is often tempting to reduce the time dedicated to the measurements in this q-range because the counting rate is fast. However if you want to go down to $10^{-3} \mathrm{~cm}^{-1}$ for characterizing objects in a solvent, you will have to subtract an incoherent scattering level that at least of $\sim 510^{-2} \mathrm{~cm}^{-1}$ in a fully deuterated solvent, and up to $\sim 1 \mathrm{~cm}^{-1}$ in a fully hydrogenated solvent ! In this case, long times of measurements are required on this q-range.

\subsubsection{Memo}

I sum up here all the standards that have to be measured for a full data reduction. All of these standards have to be measured for each configuration.

Intensities: sample, empty beam, background, empty container for sample (if necessary), flat sample ( $\mathrm{H}_{2} \mathrm{O}$ or vanadium), empty cell for $\mathrm{H}_{2} \mathrm{O}$, solvent (if necessary).

Transmissions: sample, empty beam, background, empty container for sample (if necessary), flat sample $\left(\mathrm{H}_{2} \mathrm{O}\right.$ or vanadium), empty cell for $\mathrm{H}_{2} \mathrm{O}$, solvent (if necessary).

Flux of the incident beam for obtaining absolute units, and therefore the standards enabling the determination of the attenuation factor of the incoming beam (attenuated graphite/non attenuated graphite).

In practice some beamtime can be saved in two situations. First, it is not necessary to measure the solvent scattering $\mathrm{I}_{\text {solv }}(\mathrm{q})$ at all configurations because it has a constant value over q. Only its measurement at the largest q, where the signal-to-noise ratio is good because the incoming flux is important, is necessary. Second, it is almost impossible to measure the signal of the flat sample for normalization at very small $\mathrm{q}$ because the incoming flux is too low. In this case the standards for normalization of another configuration can be used: $(i)$ when only the wavelength has been changed from one configuration to the other; or (ii) if the distance is changed keeping the wavelength constant wavelength, when the empty beam is exactly at the center of the detector. Obviously here the normalized intensity will be shifted by a factor corresponding to the ratio of fluxes between the two configurations and has to be rescaled on the scattered true intensity of an other configuration.

\subsection{Access to the beamtime and deuteriation facilities}

As SANS is probably the most popular neutron technique along with diffraction, there are several SANS spectrometers in all of the large neutron facilities. In France, there exist two neutron facilities, the Institut Laue Langevin (ILL) in Grenoble which is a European source run jointly by France, Germany and United Kingdom (41) and the Laboratoire Léon Brillouin (LLB), the French national source which is located in Saclay $30 \mathrm{~km}$ from Paris (42). There are actually 3 SANS spectrometers at ILL (D11 with a very broad accessible q-range, D22 and the very recent D33 with polarized options for magnetic studies) and 3 at LLB (PAXY and PACE for conventional studies and TPA dedicated to measurements at very small q's (9)). A new multi-purposes named PA20 with polarized option SANS spectrometer is actually under development at LLB and will open in 2015 (43).

All of these spectrometers are available free of charge by the whole scientific community. The beamtime is allocated by a committee of experts on the basis of proposals experiments. The proposals 
can generally be submitted twice a year (the deadlines varying from one facility to another). Information on formalities claims experience can be found on the websites of the facilities. All results obtained with allocated beamtime must be published without restriction. Despite the rather large offer provided by all the available spectrometers, the high demand makes their access often difficult.

It is also possible to perform experiments whose results will remain confidential, for industrial applications for example. The beamtime is no longer free and the contract allowing access to the beam must be negotiated directly with the facilities.

Finally, although neutron scattering is poorly taught at university, there exist some neutron schools open to pHD students, post-doc fellows and academic researchers with practicals on the spectrometers that enable to get familiar with SANS. In France, there are two recognized recurrent formations: Hercules which combines X-rays and neutrons in association with several facilities (in English) (44) and les FANs du LLB specifically on neutrons at LLB (in French) (42).

In many experiments, the deuteration of one of the components of the system to study is necessary for the good completion of the SANS experiment. Many molecules can be purchased from chemical manufacturers (almost all organic compounds $\S$ and many common polymers), otherwise deuteriation has to be done specifically for the experiment, either from synthesis starting from deuterated precursor or by exchanging ${ }^{1} \mathrm{H}$ with ${ }^{2} \mathrm{H}$ using tricks (such as dialysis or putting the hydrogenated molecule in saturated vapor pressure of $\mathrm{D}_{2} \mathrm{O}(42)$ ). It is always good to have a friendly chemist in the neighborhood! For biological molecules (proteins, biopolymers...), deuteriation is a very heavy task as it requires usually two difficult and tedious stepssks: the growth of living bacteria in deuterated media followed by the purification of the targeted molecule from the bacteria. In order to promote the use of neutrons for biology, two deuteration facilities are available for users, the Deuteration Laboratory at EMBL in Grenoble newt to ILL (46) and the Deuteration National Facility at ANSTO in Australia (47). The access procedures are described on their respective websites.

I want first to warmly thank Annie Brûlet that has carefully read back this course and gave me very good corrections and suggestions. Second, I want to stress that this is not an easy task to write a SANS course as there exists already plenty of excellent courses and textbooks on the topic. I did obviously not try to reinvent the technique and I was strongly inspired by the existing courses from the SFN Collection written by my colleagues, or former colleagues, from LLB Didier Lairez, Annie Brûlet and Loïc Auvray, as well as by the course of Luc Belloni. I am thus very greatful to them for having written such excellent courses! I want also to thank the organizers of the "Neutrons and Materials for Energy" school Monica Ceretti, Marie-Hélène Mathon, Clemens Ritter and Werner Paulus for their kind invitation to participate to this special issue, although I'm not myself a specialist of materials for energy.

\section{References}

[1] M. Hennion, I. Mirebeau. Diffusion de neutrons aux petits angles (DNPA) et magnétisme: concepts et applications. Journal de Physique IV 1999, Vol. Prl, 51-66.

[2] F. Ott, Neutron scattering on magnetic nano-objects. Collection SFN. Vol. 13, 02005.

[3] A. Guinier, G. Fournet. Small Angle Scattering of X-Rays. New York : Wiley, 1955.

[4] Neutrons, $X$-rays and light: scattering methods applied to soft condensed matter. Editors: T Zemb, P Lindner, North Holland, 2002.

[5] B. Hammouda, Probing Nanoscale Structures - The SANS Toolbox. www.ncnr.nist.gov/ staff/hammouda/the_ sans_ toolbox.pdf.

[6] I. Grillo, I. Small-Angle Neutron Scattering and Applications in Soft Condensed Matter pp723782 in Soft Matter Characterization. Editors: R. Pecora, R. Borsali., Springer, 2008.

$\S \S$ Deuterated solvents are indeed routinely used in laboratory techniques such as Infra Red spectroscopy or ${ }^{2} \mathrm{H}$ NMR. 
[7] The home for Small Angle Scattering. http://smallangle.org/content/small-anglescattering-bibliography.

[8] Société Française de la Neutronique. Cours des Ecoles Thématiques. http://www.sfn.asso. fr/ecoles-thematiques/.

[9] S. Désert, V. Thévenot, J. Oberdisse, A. Brûlet. The new very-small-angle neutron scattering spectrometer at Laboratoire Léon Brillouin. J. Appl. Cryst. 2007, Vol. 40, s471-s473.

[10] F. Cousin, J. Gummel, D. Clemens, I. Grillo, F. Boué. Multiple Scale Reorganization of Electrostatic Complexes of PolyStyreneSulfonate and Lysozyme. Langmuir. 2010, Vol. 26(10), 7078-7085.

[11] F. Cousin, J. Gummel, S. Combet, F. Boué. The model Lysozyme-PSSNa system for electrostatic complexation: Similarities and differences with complex coacervation. Advances in Colloid and Interface Science. 2011, Vol. 167, 71-84.

[12] D. Lairez, J. Pelta. Diffusion de neutrons aux petits angles: application à l'étude des macromolécules biologiques en solution. Journal de Physique IV. 2005, Vol. 130, 39-62.

[13] F. Muller, S. Manet, B. Jean, J.-P. Chambat, F. Boué, L. Heux, F. Cousin. F. Muller, S. Manet, B. Jean, J.-P. CSANS Measurements of Semi-Flexible Xyloglucan Polysaccharide Chains in Water Reveal Their Self-avoiding Statistics. Biomacromolecules. 2011, Vol. 12, 3330-3336.

[14] SasView for Small Angle Scattering Analysis. http://sasview.org/index.html.

[15] J. Kohlbrecher, I. Bressle. Software package SASfit for fitting small-angle scattering curves. https://kur.web.psi.ch/sans1/SANSSoft/sasfit.html.

[16] J. Teixeira, Small-Angle Scattering by Fractal Systems. J. Appl. Cryst. . 1988, Vol. 21, 781-785.

[17] D.I.Svergun, Restoring Low Resolution Structure of Biological Macromolecules from Solution Scattering Using Simulated Annealing. Biophysical Journal. 1999, Vol. 76(6), 2879-2886.

[18] L. Auvray, A. Brûlet. Diffusion de neutrons aux petits angles appliquée aux études d'interfaces et de systèmes confinés. Collection SFN. 2007, Vol. 8, 179-205 .

[19] L. Belloni, La Diffusion de Neutrons aux Petits Angles: mécanique statistique des liquides et traitement des données. Collection SFN. 2010, Vol. 11, 71-88.

[20] F. Cousin, E. Dubois, V. Cabuil. Tuning the interactions of a magnetic colloidal suspension. Phys Rev E. 2003, Vol. 68, 021405.

[21] Neutrons et Simulations. Collection SFN. 2012, Vol. 12.

[22] J. B. Hayter, J. Penfold. Self-consistent structural and dynamic study of concentrated micelle solutions. J. Chem. Soc., Faraday Trans. 1. 1981, Vol. 77, 1851-1863.

[23] Lin, M. Y., et al., et al. Universal diffusion-limited colloid aggregation. Journal of PhysicsCondensed Matter. 1990, Vol. 2(13), 3093-3113.

[24] Lin, M. Y., et al., et al. Universal reaction-limited colloid aggregation Physical Review A 1990, 41 (4), 2005-2020. Physical Review A. 1990, Vol. 41(4), 2005-2020.

[25] A. Mohraz, D.B. Moler, R.M. Ziff, M.J. Solomon. Effect of monomer geometry on the fractal structure of colloidal rod aggregates. Phys. Rev. Lett. 2004, Vol. 92, 155503.

[26] A. S. Robbes, J. Jestin, F. Meneau, F. Dalmas, O. Sandre, J. Perez, F. Boué, F. Cousin. Homogeneous dispersion of magnetic nanoparticles aggregates in a ps nanocomposite: Highly reproducible hierarchical structure tuned by the nanoparticles' size. Macromolecules. 2010, Vol. 43(13), 5785-5796.

[27] F. S. Bates, G. D. Wignall. Non-ideal mixing in binary blends of perdeuterated and protonated polystyrene. Macromolecules. 1986, Vol. 19(3), 932-934.

[28] J.P. Cotton, DNPA: introduction et variations sur le contraste. Le Journal de Physique IV. 1999, Vol. Pr1, 21-49.

[29] C. Chevigny, D. Gigmes, D. Bertin, J. Jestin, F. Boué. Polystyrene grafting from silica nanoparticles via nitroxide-mediated polymerization (nmp): synthesis and sans analysis with the contrast variation method. Soft Matter. 2009, Vol. 5(19), 3741-3753. 
[30] A.-S. Robbes, F. Cousin, F. Meneau, C. Chevigny, D. Gigmes, J. Fresnais, R.Schweins, J. Jestin. Controlled grafted brushes of Polystyrene on Magnetic gamma-Fe203 Nanoparticles via controlled Nitroxide-Mediated Polymerization. Soft Matter. 2012, Vol. 8, 3407-34.

[31] F. Cousin, J. Gummel, D. Ung, F. Boué. Polyelectrolyte-protein complexes: structure and conformation of each specie revealed by SANS. Langmuir. 2005, Vol. 21(21), 9675-9688.

[32] J. Gummel, F. Cousin, F.Boué. Counterions release from electrostatic complexes of polyelectrolytes and proteins of opposite charge: a direct measurement. JACS. 2007, Vol. 23, 7888-7098.

[33] J. Gummel, F.Cousin, F. Boué. Structure transition in PSS-lysozyme complexes: a conformation driven process, as directly seen by Small Angle Neutron Scattering . Macromolecules. 2008, Vol. 41(8), 2898-2907.

[34] N. Jouault, F. Dalmas, S. Said, E. Di Cola, R. Schweins, J. Jestin, et F. Boué. N. Jouault, F. Dalmas, S. Said, E. Di Cola, R. Schweins, J. Jestin, and F. Boue. Direct measurement of polymer chain conformation in well-controlled model nanocomposites by combining Sans and Saxs. Macromolecules. 2010, Vol. 43(23), 9881-9891.

[35] D. Lairez, Résolution d'un spectromètre de diffusion de neutrons aux petits angles. Journal de Physique IV. 1999, Vol.p. Prl-67, 67-81.

[36] D. Lairez, Bonnes pratiques de la diffusion de neutrons aux petits angles. Collection SFN. 2010, Vol. 11, 43-69.

[37] P. Lindner, R. Schweins. The D11 Small-Angle Scattering Instrument: A New Benchmark for SANS. Neutron News. 2010, Vol. 2, 15-18.

[38] Jacrot, B. The study of biological structures by neutron scattering from solution. Rep. Prog. Phys. . 1976, Vol. 39, 911-953.

[39] A. Brûlet, D. Lairez, A. Lapp, J.-P. Cotton. Improvement of data treatment in small-angle neutron scattering. J. Appl. Cryst. 2007, Vol. 40, 165-177.

[40] D. Lairez, http://didier.lairez.fr/pasimat/index.html.

[41] http://www.ill.eu/.

[42] http://www-llb.cea.fr/.

[43] G.Chaboussant, S. Désert, P. Lavie, A. Brûlet. PA20: A new SANS and GISANS project for soft matter, materials and magnetism. Journal of Physics: Conference Series. 2012, Vol. 340, 012002.

[44] http://hercules-school.eu/.

[45] Y. Nishiyama, A. Isogai, T. Okano, M. Mueller, H. Chanzy. Intracrystalline deuteration of native cellulose. Macromolecules. 1999, Vol. 32, 2078-2081.

[46] http://www.ill.eu/sites/deuteration/http://www.ill.eu/sites/deuteration/.

[47] http://www.ansto.gov.au/Research.

[48] J. Combet, Structure des solutions de polyélectrolytes: apport de la diffusion des rayons X et des neutrons aux petits angles. Collection SFN 11. 2010, Vol. 11, 153-176. 